Atomic Data and Nuclear Data Tables, 72, (1999) p.129 - 151.

ISSN: 0092-640x ISSN (electronic): 1090-2090

doi:10.1006/adnd.1999.0811

(C) 1999 Academic Press All rights reserved.

http://www.elsevier.com/wps/find/homepage.cws_home

http://www.elsevier.com/locate/adt

\title{
OSCILLATOR STRENGTHS FOR DIPOLE-ALLOWED FINE-STRUCTURE TRANSITIONS IN Fe XIII
}

\author{
SULTANA N. NAHAR \\ Department of Astronomy, The Ohio State University Columbus, Ohio 43210
}

Oscillator strengths, line strengths, and transition probabilities for fine-structure levels in silicon-like iron, Fe XIII, are reported. The data obtained are for 1223 LS bound terms, 64,456 LS multiplets, and 307,863 fine-structure transitions. Calculations are carried out in $L S$ coupling using the close coupling $R$-matrix approximation with a 14-term eigenfunction expansion. The fine-structure components are obtained through algebraic transformations. Present data considerably exceed the observed and the previously calculated data available, including those from the Opacity Project. Comparisons with previously measured and calculated values are made.

\section{INTRODUCTION}

Iron is an important element as it exists in astrophysical spectra through all ionization stages. Fe XIII, a silicon-like ion, is abundant in many astronomical sources radiating primarily in the extreme ultraviolet. Complete analysis of astrophysical spectra requires a large number of transitions in the radiative-collisional models. Comparatively few studies have been carried out for this ion. The complexities involved are due to the electron-electron correlation effects and the relatively high ion charge, which result in closely spaced bands of energy levels. There are 36 measured bound levels, corresponding to $24 \mathrm{LS}$ terms, below the ionization threshold [1]. Among the previous theoretical studies are multi-configuration Dirac-Fock calculations by Huang [2], Hartree-Fock calculations by Fawcett et al. [3], Hartree-Fock calculations including relativistic effects by Bromage et al. [4] and by Fawcett [5], and multi-configuration Thomas-Fermi calculations by Kastner et al. [6]. The lifetime of one single level, $3 s 3 p^{33} S_{1}{ }^{0}$, has been measured by Träbert et al. [7]. Previous works have been compiled in several National Institute of Standards and Technology (NIST) publications [8, 9, 10]. Under two international collaborations, the Opacity Project (OP) [11] and the Iron Project (IP) [12], efforts are being made to study the radiative and collisional processes of astrophysically abundant ions in detail. The first extensive calculations for Fe XIII were carried out by Butler et al. [13] under the OP [11] using a nine-term 
wave function expansion in the close coupling (CC) approximation using the $R$-matrix method. Their results have not yet been published, but the oscillator strengths ( $f$-values) obtained in $L S$ coupling are available through the OP database TOPbase [14].

For applications in astrophysical spectral analysis, and for comparison with laboratory plasma experiments, fine-structure data rather than $L S$ multiplet data as obtained under the OP are needed. The aim of the present work is to provide a reasonably complete set of transition probabilities for fine-structure transitions through large scale $a b$ initio calculations using the CC $R$-matrix method. Similar results for atomic transition probabilities have been obtained for other silicon-like ions, Si I, S III, Ar V, and Ca VII [15], and for iron ions, Fe II [16], Fe III [17], and Fe XXIV and Fe XXV [18]. A complementary set of data for the collision strengths for Fe XIII is being computed under the IP [12].

\section{Theory and Computations}

For a bound-bound transition from an initial state $i$ to a final state $j$, the line strength, $S$, is defined (for example, in Ref. [11]) as

$$
S=\left|\left\langle\Psi_{j}|| \mathbf{D}|| \Psi_{i}\right\rangle\right|^{2}
$$

in atomic units (a.u.), where the dipole operator $\mathbf{D}$ is

$$
\mathbf{D}=\sum_{n} \mathbf{r}_{n},
$$

in the length form summed over the total number of electrons in the ion, and $\Psi_{i}$ and $\Psi_{j}$ are the initial and the final wave functions, respectively. The line strength $S$ in atomic units is related to the oscillator strength $f_{i j}(f$-value) as

$$
S=\left(3 g_{i} / E_{i j}\right) f_{i j},
$$

where $E_{i j}$ is the transition energy in rydberg and $g i$ is the statistical weight factor of the initial state, with $g_{i}=\left(2 S_{i}+1\right)\left(2 L_{i}+1\right)$ for an $L S$ multiplet, and $\left(2 J_{i}+1\right)$ for a fine-structure transition. The transition probability, $A_{j i}$ (Einstein $A$-coefficient), can be obtained from the oscillator strength as

$$
A_{j i}(\text { a.u. })=\frac{1}{2} \alpha^{3} \frac{g_{i}}{g_{j}} E_{i j}^{2} f_{i j}, \quad A_{j i}\left(\mathrm{~s}^{-1}\right)=\frac{A_{j i}(\text { (a.u. })}{\tau_{0}},
$$

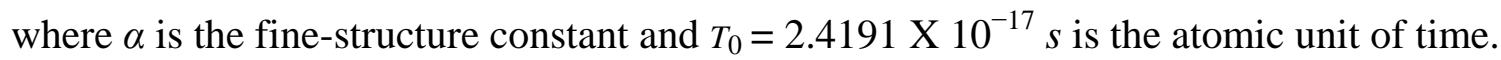

In the present work, oscillator strengths in $L S$ coupling are obtained in the CC $R$-matrix method similar to the OP work [11]. The fine-structure components of the transitions are obtained from $L S$ multiplets through algebraic transformations. The transformations can be carried out via the line strength or directly from the oscillator strength [16]. The line strength, which is 
independent of the transition energy $E_{i j}$, is a better choice. As the observed transition energies are determined more accurately than the calculated ones, use of the former with the line strengths can provide more accurate $f_{i j}$ and $A_{j i}$. Hence, in the present calculations, the fine-structure transition probabilities are obtained from $S$-values whenever observed energies are available and from $f$-values when calculated $E_{i j}$ are used.

The fine-structure line strengths, $S_{J J}$, are obtained as

$$
S_{J J}=C\left(J_{i}, J_{j}\right) S_{L S} /\left[\left(2 S_{i}+1\right)\left(2 L_{i}+1\right)\left(2 L_{j}+1\right)\right]
$$

for the allowed transitions $(\Delta J=0, \pm 1)$; $S_{i}$ is the spin which is the same as $S j$. The values of the coefficients $C\left(J_{i}, J j\right)$ can be found in Allen [19]. The $S_{J J}$ values satisfy the condition

$$
S_{L S}=\sum_{J} S_{J J}
$$

The fine-structure $f$-values, $f_{J J}$, can be obtained directly from $f_{L S}$ as [20]

$$
\begin{aligned}
f_{J J}\left(n_{j} S_{i} L_{J} J_{j}, n_{i} S_{i} L_{i} J_{i}\right)= & f_{L S}\left(n_{j} S_{i} L_{j}, n_{i} S_{i} L_{i}\right)\left(2 J_{j}+1\right) \\
& \times\left(2 L_{i}+1\right) W^{2}\left(L_{j} L_{t} J_{j} J_{i} ; 1 S_{i}\right),
\end{aligned}
$$

where $W\left(L_{j} L_{i} J_{j} J_{i} ; 1 S_{i}\right)$ is a Racah coefficient. The above values also satisfy the sum rule

$$
\begin{aligned}
\sum_{J_{J} J_{j}}\left(2 J_{i}+1\right) & f_{J J}\left(n_{j} S_{i} L_{j} J_{j}, n_{i} S_{i} L_{i} J_{i}\right) \\
= & \left(2 S_{i}+1\right)\left(2 L_{i}+1\right) f_{L S}\left(n_{j} S_{i} L_{j}, n_{i} S_{i} L_{i}\right) .
\end{aligned}
$$

The above form is used when one or both $L S$ terms of the transition are unobserved or when not all the fine structure levels of an $L S$ term are observed, and for transitions between high angular momentum states (transitions involving terms higher than $H \leftrightarrow I$ where Allen's coefficients are not available).

The lifetime, $\tau_{j}$, of a state or level $j$ can be obtained from the transition probabilities to the lower levels, that is, from the $A$-values, as

$$
\tau_{j}=\frac{1}{A_{j}\left(\mathrm{~s}^{-1}\right)}
$$

where $A_{j}$ is the total radiative transition probability for the state or level $j$, that is,

$$
A_{j}=\sum_{i} A_{j i}
$$

In the CC approximation the total wave function of the ion, $\Psi(E)$, is an expansion of core states, $\mathrm{X}_{i}$. The core is termed the "target" of $\mathrm{N}$ electrons. For any symmetry $S L \pi$, 


$$
\Psi(E)=\mathbf{A} \sum_{i} \chi_{i} \theta_{i}+\sum_{j} c_{j} \Phi_{j},
$$

where $\chi_{i}$ is a specific target state $S_{i} L_{i} \pi_{i} ; \theta_{i}$ is the wave function of the $(N+1)$ th electron in a channel labeled $S i L i \pi_{i} k i_{i}(S L \pi)$ where $k_{i}^{2}$ is the electron energy, which for $k_{i}^{2}<0$ may represent a bound state of the electron-ion system; and $\mathbf{A}$ is the antisymmetrization operator. The $\Phi_{j}$ 's are correlation functions of the $(N+1)$ th electron system that compensate for the orthogonality condition of the total wave function as well as account for short-range correlation effects, and the $c_{j}$ 's are the variational coefficients.

The present wave function expansion of Fe XIII is a 14-term expansion consisting of the 14 lowest terms of the core Fe XIV. The terms and the energies are given in Table A. The term energies and the orbital wave functions of the core or the target ion are obtained from atomic structure calculations using the program SUPERSTRUCTURE [21]. However, the calculated energies of observed terms have been replaced by the measured values listed in the NIST compilation [1] where available. Not all the terms in Table A have been observed; the unobserved term energies are marked by asterisks. The sets of spectroscopic and correlation configurations, and the values of the scaling parameter $\lambda$ for each orbital in the Thomas-Fermi-Dirac potential used in the atomic structure calculations are given in the note to Table A. The second sum in the wave function expansion, Eq. (11), includes all possible $(N+1)$-electron configurations of Fe XIII up to $3 p^{4}, 3 d^{2}, 4 s$, and $4 p$.

TABLE A

Fe XIV States and Excitation Energies Used in the Wave Function Expansion of Fe XIII

\begin{tabular}{|c|c|c|c|c|c|c|c|}
\hline & Configuration & $S L \pi$ & $E(\mathrm{Ry})$ & & Configuration & $S L \pi$ & $E(\mathrm{Ry})$ \\
\hline 1 & $3 s^{2} 3 p$ & ${ }^{2} P^{0}$ & 0.0 & 8 & $3 p^{3}$ & ${ }^{4} S^{0}$ & 5.34121 \\
\hline 2 & $3 s 3 p^{2}$ & ${ }^{4} P^{\mathrm{e}}$ & 2.12457 & 9 & $3 p^{3}$ & ${ }^{2} P^{0}$ & $5.58566^{*}$ \\
\hline 3 & $3 s 3 p^{2}$ & ${ }^{2} D^{\mathrm{e}}$ & 2.73911 & 10 & $3 s 3 p 3 d$ & ${ }^{4} F^{0}$ & $5.63024 *$ \\
\hline 4 & $3 s 3 p^{2}$ & ${ }^{2} S^{e}$ & 3.32333 & 11 & $3 s 3 p 3 d$ & ${ }^{4} P^{0}$ & $6.07468 *$ \\
\hline 5 & $3 s 3 p^{2}$ & ${ }^{2} P^{\mathrm{e}}$ & 3.58899 & 12 & $3 s 3 p 3 d$ & ${ }^{4} D^{\circ}$ & 6.11603 \\
\hline 6 & $3 s^{2} 3 d$ & ${ }^{2} D^{\mathrm{e}}$ & 4.32324 & 13 & $3 s 3 p 3 d$ & ${ }^{2} D^{\circ}$ & $6.35966^{*}$ \\
\hline 7 & $3 p^{3}$ & ${ }^{2} D^{0}$ & $4.91648^{*}$ & 14 & $3 s 3 p 3 d$ & ${ }^{2} F^{0}$ & $6.67736^{*}$ \\
\hline
\end{tabular}

Note. The energies marked with an asterisk are calculated energies, given here for unobserved terms. Following are the sets of spectroscopic and correlation configurations, and the scaling parameters, $\mathbf{A}$, for each orbital in the Thomas-Fermi-Dirac potential used in the atomic structure calculations. 
Spectroscopic configurations: $3 s^{2} 3 p, 3 s 3 p^{2}, 3 p^{3}, 3 s^{2} 3 d, 3 s 3 p 3 d, 3 p^{2} 3 d$.

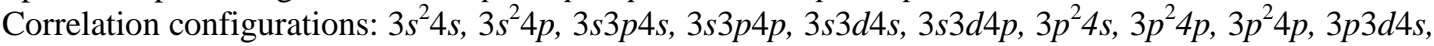

$3 p 3 d 4 p$.

$\lambda$ (Scaling parameters): 1.1(1s), 1.08576(2s), 1.03329(2p), 0.947(3s), 0.90957(3p), 1.00689(3d),

4.85982(4s), 2.19876(4p).

The computations of the oscillator strengths for bound-bound transitions are carried out using the $R$-matrix package of codes developed for the OP [22] and extended for the IP [12]. Computations include all $S L \pi$ of Fe XIII formed from the target states in combination with the outer electron with $l \leq 9$. The fine-structure components of the $f$-values are obtained using the code JJTOLS [16]. Due to the large volume, computations and processing of data are carried out separately for each spin symmetry $(2 S+1)=5$, 3 , and 1 .

All bound states of the Hamiltonian matrix are scanned for up to $n=10$ and $l=9$ with an effective quantum number mesh $\Delta v=0.01$. Identification of the large number of $L S$ bound states obtained has been a major task for this ion. A term is designated as a possible combination of the configuration of the core and the outer electron, corresponding to an appropriate value of $v$ for the outer electron quantum numbers $n, l$ and a larger channel percentage contribution. One way to identify the states is to sort out the series of $v$ for the terms of same configuration but with increasing $n$ of the valence electron. However, small differences in $v$ for various terms of the same symmetry have caused difficulties in assignments of proper configurations. The quantum defects for these states are almost the same. Hence, some uncertainties may have been introduced in the identification of some of the states; that is, the exact identification could also be the immediate upper or lower term for such cases. Almost all the bound terms of Fe XIII are formed from the 14 Fe XIV target states included in combination with the outer electron quantum numbers. However, some additional bound states are found to have formed from the bound channel configurations included (second sum of Eq. (11)) in the calculations. These terms are assigned with possible configurations with core of the forms $3 s 3 d^{2}, 3 p 3 d^{2}$, and $3 d^{2} 4 s$.

\section{Results and Discussion}

The 14 CC $R$-matrix calculations have resulted in a total of 1223 bound $L S$ terms of Fe XIII lying below the first ionization threshold. Of these, 24 states have been observed [1] and identified. The calculated term energies are compared with the observed ones in Table B. The earlier calculations [13] under the OP correspond to a 9-term eigenfunction expansion leading to 797 bound terms. In Table B, a comparison of present calculated energies with those of Ref. [13], and with observed energies, shows good agreement among the different values, generally to within $1 \%$.

Table I lists the complete set of bound LS terms and energies among which the dipole-allowed fine-structure transitions are considered. In the Table, the calculated energies of the 24 observed terms have been replaced by the measured values, as these are used in the calculation of transition probabilities. Each $L S$ term is prefixed by a degeneracy symbol, a letter of the alphabet, for convenience of identification. Ascending order of the alphabet is chosen for the even-parity states of a symmetry. The odd-parity states are designated in the same way but with the alphabet in descending order. No degeneracy is assigned for terms in a position 27th or higher within the symmetry.

The dipole-allowed bound-bound transitions in Fe XIII result in 64,456 transitions among 
$L S$ terms. The present number of transitions is over $71 \%$ more than the number of transitions, 37,598, obtained by Butler et al. [13]. Fine-structure transitions have been derived for all $L S$ multiplets in the present work, yielding an extensive set of 307,863 transitions. Observed energies have been used wherever available for improved accuracy.

Present $f$-values, both for $L S$ multiplets and for fine-structure transitions, are compared with those in the previous calculations in Table C. With the exception of the calculation by Butler et al. [13], who carried out CC

TABLE B

Comparison of Calculated Fe XIII Binding Energies, $E_{c}$ (in rydberg)

with the Observed Values, $E_{o}$ [1], and with Calculated Values

$E_{c}(\mathrm{OP})$ from [13] 


\begin{tabular}{|c|c|c|c|c|}
\hline Configuration & $S L \pi$ & $\begin{array}{c}E_{0} \\
N_{0}=24\end{array}$ & $\begin{array}{c}E_{c} \\
N_{c}=1223\end{array}$ & $\begin{array}{c}E_{c}(\mathrm{OP}) \\
N_{c}=797\end{array}$ \\
\hline $3 s^{2} 3 p^{2}$ & ${ }^{3} P$ & 26.414 & 26.393 & 26.345 \\
\hline $3 s^{2} 3 p^{2}$ & ${ }^{1} D$ & 26.098 & 26.107 & 26.064 \\
\hline $3 s^{2} 3 p^{2}$ & ${ }^{1} S$ & 25.702 & 25.701 & 25.643 \\
\hline $3 s 3 p^{3}$ & ${ }^{3} D^{0}$ & 23.906 & 24.029 & 23.985 \\
\hline $3 s 3 p^{3}$ & ${ }^{3} P^{0 *}$ & 23.529 & 23.657 & 23.608 \\
\hline $3 s 3 p^{3}$ & ${ }^{1} D^{\circ}$ & 23.234 & 23.339 & 23.301 \\
\hline $3 s 3 p^{3}$ & ${ }^{3} S^{0}$ & 22.750 & 22.781 & 22.735 \\
\hline $3 s 3 p^{3}$ & ${ }^{1} P^{0}$ & 22.544 & 22.652 & 22.612 \\
\hline $3 s^{2} 3 p 3 d$ & ${ }^{3} P^{0}$ & 22.061 & 22.029 & 21.976 \\
\hline $3 s^{2} 3 p 3 d$ & ${ }^{1} D^{\circ}$ & 21.990 & 22.068 & 22.014 \\
\hline $3 s^{2} 3 p 3 d$ & ${ }^{3} D^{0}$ & 21.901 & 21.935 & 21.881 \\
\hline $3 s^{2} 3 p 3 d$ & ${ }^{1} F^{0}$ & 21.462 & 21.461 & 21.417 \\
\hline $3 s^{2} 3 p 3 d$ & ${ }^{1} P^{0}$ & 21.336 & 21.348 & 21.286 \\
\hline $3 s^{2} 3 p 4 s$ & ${ }^{3} P^{0 *}$ & 14.254 & 14.155 & 14.172 \\
\hline $3 s^{2} 3 p 4 s$ & ${ }^{1} P^{0}$ & 14.126 & 14.034 & 14.084 \\
\hline $3 s^{2} 3 p 4 p$ & ${ }^{1} D$ & 12.975 & 12.810 & 12.960 \\
\hline $3 s^{2} 3 p 4 p$ & ${ }^{1} P$ & 12.728 & 12.424 & 13.336 \\
\hline $3 s^{2} 3 p 4 d$ & ${ }^{3} D^{0}$ & 11.907 & 11.854 & 11.852 \\
\hline $3 s^{2} 3 p 4 d$ & ${ }^{3} F^{0} *$ & 11.777 & 11.767 & 11.767 \\
\hline $3 s^{2} 3 p 4 d$ & ${ }^{1} F^{0}$ & 11.677 & 11.705 & 11.695 \\
\hline $3 s^{2} 3 p 4 d$ & ${ }^{1} P^{0}$ & 11.495 & 11.641 & 11.630 \\
\hline $3 s^{2} 3 p 4 f$ & ${ }^{1} D$ & 10.673 & 10.644 & 10.641 \\
\hline $3 s^{2} 3 p 4 f$ & ${ }^{3} F^{*}$ & 10.668 & 10.919 & 10.920 \\
\hline $3 s^{2} 3 p 4 f$ & ${ }^{1} G$ & 10.648 & 10.661 & 10.653 \\
\hline
\end{tabular}

Note. An asterisk indicates incomplete set of observed fine-structure levels. $N$ is the total number of bound states.

$R$-matrix calculations, other previous works correspond to atomic structure calculations requiring optimization of individual levels. A relatively smaller number of transitions, including both dipole allowed and intercombination, were considered in these calculations. All calculations agree better for the singlet transitions than for the triplet ones. Present LS oscillator strengths are consistently in good agreement with those given by Butler et al. [13] for most of the transitions. However, significant differences also exist for a few cases, such as for the transition $3 s^{2} 3 p^{2}\left({ }^{3} P\right) \rightarrow$ $3 s^{2} 3 p 4 s\left({ }^{3} P^{0}\right)$. The term energies for this transition from both calculations are in good agreement (Table B); the reason for the large difference in the $f$-value is not obvious but could be related to correlation effects in the present work. Agreement is quite good for the present fine-structure $f$-values with those obtained using multiconfiguration Dirac-Fock calculations by Huang [2] for the dipole-allowed transitions, $3 s^{2} 3 p^{2}\left({ }^{1} S,{ }^{1} D\right) \rightarrow 3 p 3 d\left({ }^{1} P^{0},{ }^{1} F^{0}\right)$, and for $3 s^{2} 3 p^{2}\left({ }^{3} P\right) \rightarrow 3 p 3 d\left({ }^{3} P^{0}\right.$, $\left.{ }^{3} D^{0}\right)$, but the differences are considerable for the transitions $3 s^{2} 3 p^{2}\left({ }^{3} P\right) \rightarrow 3 s 3 p^{3}\left({ }^{3} P^{0},{ }^{3} D^{0}\right)$. The disagreement with [2] shown for the $3 s^{2} 3 p^{2}\left({ }^{3} P\right) \rightarrow 3 s 3 p^{3}\left({ }^{3} S^{0}\right)$ transition may be spurious;

rather good agreement is actually seen if the $3 s 3 p^{3}\left({ }^{3} S^{0}\right)$ and $3 s 3 p^{3}\left({ }^{1} P^{0}\right)$ state labels are switched in [2]. Varying degrees of agreement with the oscillator strengths are found by Fawcett and collaborators (Fawcett et al. [3], Bromage et al. [4], and Fawcett [5]). While present values agree very well with those given by Fawcett et al. [3] for transitions such as $3 s^{2} 3 p^{2}\left({ }^{1} D\right) \rightarrow 3 p 4 s\left({ }^{1} P^{0}\right)$, $3 p 3 d\left({ }^{3} F^{0}\right) \rightarrow 3 p 4 p\left({ }^{3} D\right)$, the agreement is quite poor for some other transitions such as for $3 s^{2} 3 p^{2}\left({ }^{3} P\right) \rightarrow 3 p 4 s\left({ }^{3} P^{0}\right)$. The Dirac-Fock calculations by Huang [2] and the Hartree-Fock calculations including relativistic effects by Fawcett and collaborators often are in good agreement with each other. The results from these atomic structure calculations can be very accurate for some transitions but they can be considerably poorer without suitable optimization. In contrast, in the 
CC approximation, such as that used in the present case, the results are of consistent accuracy throughout except for very weak transitions. However, for transitions where relativistic effects are more important, the atomic structure calculations including these effects are probably more accurate. This is especially the case for transitions affected by relativistic mixing between different multiplicities, such as between the singlets and triplets. In recent developments under the IP [12] it is now possible to carry out CC calculations including the relativistic effects using the Breit-Pauli approximation; this method has been used for the transition probabilities for two simple systems, Fe XXIV and Fe XXV [18]. Carrying out of such calculations for Fe XIII is planned.

As the lifetime of the $3 s 3 p^{3}\left({ }^{3} S_{1}^{0}\right)$ level of Fe XIII has been measured experimentally by Träbert et al. [7], it is of considerable interest to study the decay rate from this level, namely the transition $3 s^{2} 3 p^{2}\left({ }^{3} P\right) \leftarrow 3 s 3 p^{3}\left({ }^{3} S^{0}\right)$. The $f$-values from the three calculations, Refs. [13], and [4] and the present, agree in general for the $L S$ transition (Table C), indicating similar predictions for the lifetime (Table D). However, the slight variation in the calculated energies will introduce some differences. The lifetime values from previous calculations are 16.4 ps [13], 16.4 ps [4] (from the $L S A$-value), and 16.7 ps [4] (from fine-structure components), and the value from the present calculation is 15.8 ps (as shown in Table D). The measured value is 21(4) ps with uncertainty ranging from 17 to 25 ps [7]. The calculated values agree with one another within the expected uncertainty of the various theoretical treatments, and they agree with the lower limit of the measured value, $17 \mathrm{ps}$, to within a few percent. However, it may be that all theoretical values somewhat underestimate the lifetime of the ${ }^{3} S^{0}$ level.

Based on the accuracy of the calculated term energies in comparison with the measured values, of the present $f$-values with previous calculations and of the lifetime with the measured value, and on the general uncertainty of the CC method, it is estimated that the accuracy of the present $f-, S$-, and $A$-values is approximately $10-30 \%$ for most transitions. The uncertainty can be higher for weak transitions.

TABLE C Comparison of Fe XIII $f$-Values 


\begin{tabular}{|c|c|c|c|c|c|}
\hline Transition & Multiplet & $g_{i}$ & $g_{f}$ & Present & Others \\
\hline $3 s^{2} 3 p^{2}-3 s 3 p^{3}$ & ${ }^{1} S-{ }^{1} P^{0}$ & 1 & 3 & 0.186 & $0.175[13], 0.15[4], 0.138[5]$ \\
\hline $3 s^{2} 3 p^{2}-3 p 3 d$ & ${ }^{1} S-{ }^{1} P^{0}$ & 1 & 3 & 1.10 & $1.12[13], 1.1[2], 1.2[4], 1.26[5]$ \\
\hline $3 s^{2} 3 p^{2}-3 p 4 d$ & ${ }^{1} S-{ }^{1} P^{0}$ & 1 & 3 & 0.534 & $0.524[13], 0.39[6]$ \\
\hline $3 s^{2} 3 p^{2}-3 s 3 p^{3}$ & ${ }^{1} D-{ }^{1} P^{0}$ & 5 & 3 & 0.183 & 0.179 [13], $0.18[4], 0.189[5]$ \\
\hline $3 s^{2} 3 p^{2}-3 p 4 s$ & ${ }^{1} D-{ }^{1} P^{0}$ & 5 & 3 & 0.12 & $0.11[13], 0.11[3]$ \\
\hline $3 s^{2} 3 p^{2}-3 s 3 p^{3}$ & ${ }^{1} D-^{1} D^{\circ}$ & 5 & 5 & 0.0967 & $0.0918[13], 0.080[4], 0.077[5]$ \\
\hline $3 s^{2} 3 p^{2}-3 p 3 d$ & ${ }^{1} D-{ }^{1} D^{\circ}$ & 5 & 5 & 0.443 & $0.444[5]$ \\
\hline $3 s^{2} 3 p^{2}-3 p 3 d$ & ${ }^{1} D-{ }^{1} F^{\circ}$ & 5 & 7 & 0.578 & $0.582[13], 0.55[2], 0.58[4], 0.58[5]$ \\
\hline $3 s^{2} 3 p^{2}-3 p 4 d$ & ${ }^{1} D-{ }^{1} F^{0}$ & 5 & 7 & 0.384 & $0.382[13], 0.33[3,6]$ \\
\hline $3 p 3 d-3 p 4 p$ & ${ }^{1} F^{0}-{ }^{1} D$ & 7 & 5 & 0.0306 & $0.0301[13], 0.22[3]$ \\
\hline $3 p 3 d-3 p 4 f$ & ${ }^{1} F^{0}-{ }^{1} G$ & 7 & 5 & 0.72 & $0.71[13], 0.75[3,6]$ \\
\hline \multirow[t]{4}{*}{$3 s^{2} 3 p^{2}-3 s 3 p^{3}$} & ${ }^{3} P-{ }^{3} S^{0}$ & 9 & 3 & 0.196 & $0.194[13], 0.19[4]$ \\
\hline & & 5 & 3 & 0.194 & $0.005[2], 0.20[4], 0.195[5]$ \\
\hline & & 3 & 3 & 0.198 & $0.042[2], 0.16[4], 0.162[5]$ \\
\hline & & 1 & 3 & 0.203 & $0.019[2], 0.18[4], 0.162[5]$ \\
\hline \multirow{7}{*}{$3 s^{2} 3 p^{2}-3 s 3 p^{3}$} & ${ }^{3} P-{ }^{3} P^{0}$ & 9 & 9 & 0.0637 & $0.0591[13], 0.057[2]$ \\
\hline & & 5 & 5 & 0.047 & $0.049[2], 0.05[5]$ \\
\hline & & 5 & 3 & 0.0156 & $0.0083[2], 0.0084[5]$ \\
\hline & & 3 & 5 & 0.027 & $0.010[2], 0.011[5]$ \\
\hline & & 3 & 3 & 0.016 & $0.027[2], 0.027[5]$ \\
\hline & & 3 & 1 & 0.021 & $0.020[2], 0.020[5]$ \\
\hline & & 1 & 3 & 0.064 & $0.051[2], 0.053[5]$ \\
\hline \multirow[t]{7}{*}{$3 s^{2} 3 p^{2}-3 s^{2} 3 p 3 d$} & ${ }^{3} P-{ }^{3} P^{0}$ & 9 & 9 & 0.283 & $0.286[13]$ \\
\hline & & 5 & 5 & 0.208 & $0.122[2], 0.127[5]$ \\
\hline & & 5 & 3 & 0.070 & $0.034[2], 0.024[4], 0.025[5]$ \\
\hline & & 3 & 5 & 0.118 & $0.213[5]$ \\
\hline & & 3 & 3 & 0.072 & $0.0001[2], 0.0053[5]$ \\
\hline & & 3 & 1 & 0.097 & $0.098[2], 0.104[5]$ \\
\hline & & 1 & 3 & 0.293 & $0.819[2], 0.97[4], 0.948[5]$ \\
\hline \multirow[t]{4}{*}{$3 s^{2} 3 p^{2}-3 p 4 s$} & ${ }^{3} P-{ }^{3} P^{0}$ & 9 & 9 & 0.0624 & $0.10[13]$ \\
\hline & & 5 & 5 & 0.0468 & $0.088[3]$ \\
\hline & & 5 & 3 & 0.0154 & $0.040[3]$ \\
\hline & & 3 & 5 & 0.0262 & $0.057[3]$ \\
\hline \multirow[t]{7}{*}{$3 s^{2} 3 p^{2}-3 s 3 p^{3}$} & ${ }^{3} P-{ }^{3} D^{0}$ & 9 & 15 & 0.052 & $0.048[13], 0.046[2]$ \\
\hline & & 5 & 7 & 0.043 & $0.036[2], 0.034[5]$ \\
\hline & & 5 & 5 & 0.0077 & $0.0010[2]$ \\
\hline & & 5 & 3 & 0.0005 & $0.00015[2]$ \\
\hline & & 3 & 5 & 0.040 & $0.048[2], 0.046[5]$ \\
\hline & & 3 & 3 & 0.013 & $0.0065[2], 0.0063[5]$ \\
\hline & & 1 & 3 & 0.055 & $0.069[2], 0.067[5]$ \\
\hline \multirow[t]{7}{*}{$3 s^{2} 3 p^{2}-3 s^{2} 3 p 3 d$} & ${ }^{3} P-{ }^{3} D^{0}$ & 9 & 15 & 0.707 & $0.704[13], 0.65[4]$ \\
\hline & & 5 & 7 & 0.59 & $0.577[2], 0.59[4], 0.57[2], 0.585[5]$ \\
\hline & & 5 & 5 & 0.105 & $0.0004[2], 0.23[4], 0.227[5]$ \\
\hline & & 5 & 3 & 0.007 & $0.047[2], 0.06[4], 0.057[5]$ \\
\hline & & 3 & 5 & 0.53 & $0.17[2], 0.19[4], 0.206[5]$ \\
\hline & & 3 & 3 & 0.18 & $0.25[2], 0.25[4], 0.255[5]$ \\
\hline & & 1 & 3 & 0.72 & $0.162[2], 0.08[4], 0.095[5]$ \\
\hline \multirow[t]{3}{*}{$3 s^{2} 3 p^{2}-3 s^{2} 3 p 4 d$} & ${ }^{3} P-{ }^{3} D^{\circ}$ & 9 & 15 & 0.361 & $0.346[13]$ \\
\hline & & 3 & 5 & 0.27 & $0.23[3]$ \\
\hline & & 1 & 3 & 0.36 & $0.35[3]$ \\
\hline \multirow[t]{4}{*}{$3 p 3 d-3 p 4 p$} & ${ }^{3} F^{0}-{ }^{3} D$ & 21 & 15 & 0.0478 & $0.0474[13]$ \\
\hline & & 9 & 7 & 0.048 & $0.046[3]$ \\
\hline & & 7 & 5 & 0.042 & $0.040[3]$ \\
\hline & & 5 & 3 & 0.040 & $0.034[3]$ \\
\hline \multirow[t]{3}{*}{$3 p 3 d-3 p 4 f$} & ${ }^{3} F^{0}-{ }^{3} G$ & 21 & 27 & 0.792 & $0.776[13]$ \\
\hline & & 9 & 11 & 0.75 & $0.73[3,6]$ \\
\hline & & 7 & 9 & 0.74 & $0.50[3]$ \\
\hline
\end{tabular}

TABLE D Lifetime (in $10^{-12} \mathrm{~s}$ ) of the $3 s 3 p^{3}\left({ }^{3} S_{1}^{O}\right)$ Level in Fe XIII 


\begin{tabular}{|c|c|c|c|c|}
\hline \multirow[b]{2}{*}{ Level } & \multirow[b]{2}{*}{ Present } & \multicolumn{2}{|c|}{ Theory } & \multirow{2}{*}{$\begin{array}{c}\text { Experiment } \\
{[7]}\end{array}$} \\
\hline & & [13] & [4] & \\
\hline $3 s 3 p^{3}\left({ }^{3} S_{1}^{o}\right)$ & 15.8 & 16.4 & $16.4^{a}, 16.7^{b}$ & $21(4)$ \\
\hline
\end{tabular}

However, more measured values and more accurate calculations are required for better estimation of the accuracy of the present values. The present method does not include any relativistic effects for the fine-structure transitions. For highly excited states, the relativistic effects and LSJ-mixing can be significant. These may result in stronger intercombination transitions and somewhat different strengths among the corresponding allowed fine-structure components. As discussed in the previous section, there are also uncertainties in configuration assignments for some of the states due to fact that the quantum defects are nearly the same. Hence, the identification of some of these transitions may not be exact.

The volume of results obtained for the oscillator strengths ( $f$ ), line strength $(S)$, and transition probabilities (A-values) for Fe XIII is quite large. It is impractical to publish such a large number of transitions. Hence, the table containing the complete set of $f$-, $S$-, and $A$-values for both $L S$ multiplets and fine-structure transitions along with 1223 energy terms will be made available electronically. A FORTRAN code will be provided to read the file and obtain the relevant quantities, such as $A$-values for lifetime calculations. Table II represents a partial table presenting only those transitions for which observed energies have been used. The format corresponds to that of the complete table.

\section{Summary and Conclusion}

An extensive set of $f$-, $S$-, and $A$-values for 64,456 LS-multiplet transitions and 307,863 dipole-allowed fine-structure transitions in Fe XIII is obtained. This work presents the first extensive data set for fine-structure transitions for this ion. The number of bound states obtained is considerably larger than for previously measured and calculated data. Calculations are carried out in the CC $R$-matrix method for the $L S$ transitions, and the fine-structure components are obtained through algebraic transformations. The observed energies are used, wherever available, for improved accuracy. The uncertainty is estimated to be $10 \%$ for strong transitions and about $10-30 \%$ for most other transitions. Inclusion of relativistic effects, which have not been

considered in the present work, could lead to further improvements. Present results should be applicable to various laboratory and astronomical plasma diagnostics, such as in the detailed analysis of UV spectra.

The full table of transition probabilities and energies is available in electronic form from the author at nahar@astronomy.ohio-state.edu. A FORTRAN77 code is attached to the table to read the $A$-values and calculate the lifetime for any $L S$ term or fine-structure level.

\section{Acknowledgments}

I thank Professor Anil K. Pradhan for comments. This work was partially supported by a grant from the NSF 
for the Iron Project and by the NASA UV and Optical Astrophysical Program. Computations were carried out on the Cray T-94 at the Ohio Supercomputer Center.

\section{References}

1. J. Sugar and C. Corliss, J. Phys. Chem. Ref. Data 14, Suppl. 2 (1985)

2. K.-N. Huang, Atomic Data AND NuClear Data TABles 32, 503 (1985)

3. B. C. Fawcett, R. D. Cowan, E. Y. Kononov, and R. W. Hayes, J. Phys. B 5, 1255 (1972)

4. G. E. Bromage, R. D. Cowan, B. C. Fawcett, Mon. Not. R. Astron. Soc. 183, 19 (1978)

5. B. C. Fawcett, Atomic Data And Nuclear Data TAbles

36, 129 (1987)

6. S. O. Kastner, M. Swartz, A. K. Bhatia, and J. Lapides, J. Opt. Soc. Am. 68, 1558 (1978)

7. E. Träbert, K. W. Jones, B. M. Johnson, D. C. Gregory, and T. H. Kruse, Phys. Lett A 87, 336 (1982)

8. J. R. Fuhr, G. A. Martin, W. L. Wiese, and S. M. Younger, J. Phys. Chem. Ref. Data 10, 305 (1981)

9. J. R. Fuhr, G. A. Martin, and W. L. Wiese, J. Phys. Chem. Ref. Data 17, Suppl. 4 (1988)

10. T. Shirai, Y. Funatake, K. Mori, J. Sugar, W. L. Wiese, and Y. Nakai, J. Phys. Chem. Ref. Data 19, 127 (1990)

11. M. J. Seaton, J. Phys. B 20, 6363 (1987)

12. D. G. Hummer, K. A. Berrington, W. Eissner, A. K. Pradhan, H. E. Saraph, and J. A. Tully, Astron. Astrophys. 279, 298 (1993)

13. K. Butler, C. Mendoza, and C. J. Zeippen (in preparation); the data are available through the Opacity Project database, TOPbase (Cunto et al. Ref. 14)

14. W. Cunto, C. Mendoza, F. Ochsenbein, and C. J. Zeippen, Astron. Astrophys. 275, L5 (1993)

15. S. N. Nahar, Physica Scripta 48, 297 (1993); more complete sets of transition probabilities for the ions can be obtained electronically from the author

16. S. N. Nahar, Astron. Astrophys. 293, 967 (1995)

17. S. N. Nahar and A. K. Pradhan, Astron. Astrophys. Suppl. Ser. 119, 509 (1996)

18. S. N. Nahar and A. K. Pradhan, Astron. Astrophys. Suppl. Ser. 135, 347 (1999)

19. C. W. Allen, Astrophysical Quantities (3rd ed.) (Athlone Press, London, 1976)

20. M. J. Seaton, Yu Yan, D. Mihalas, and A. K. Pradhan, Mon. Not. R. Astron. Soc. 266, 805 (1994)

21. W. Eissner, M. Jones, and H. Nussbaumer, Comput. Phys. Commun. 8, 270 (1974); W. Eissner, J. Phys. IV (Paris) C1, 3 (1991)

22. K. A. Berrington, P. G. Burke, K. Butler, M. J. Seaton, P. J. Storey, K. T. Taylor, and Yu Yan, J. Phys. B 20, 6379 (1987)

\section{EXPLANATION OF TABLES}


TABLE I. Bound States of Fe XIII

This Table presents the bound states of Fe XIII among which the dipole-allowed transitions are considered.

Term Electronic configuration and $L S$ term. The lower $L S$ terms are prefixed by a degeneracy symbol in alphabetically ascending order for successive even-parity states and descending order for successive odd-parity states of the same symmetry. No degeneracy is assigned for terms in a position 27th or higher within the symmetry.

E(Ry) LS binding energy (in rydberg) used in the present calculation. (Negative sign is omitted for convenience.)

TABLE II. Oscillator Strengths, Line Strengths, and Transition Probabilities for Allowed Transitions in Fe XIII

Data are given in subsets, with the first line corresponding to the $L S$ transition and subsequent lines to its fine-structure components. In the complete electronic file the subsets are ordered by observed states, as given here, followed by the calculated states.

Transition The transition $I \rightarrow j$, with states $i$ and $j$ in the notation of Table I. $E i, E j \quad$ Binding energies of the initial and final $L S$ terms given in rydberg on the first line, and the excitation energies of the initial and final fine-structure levels given in $\mathrm{cm}^{-1}$ on the subsequent lines. (The negative sign for the $L S$ term binding energy is omitted for convenience.) Asterisks in place of the fine-structure energies mean one or both levels are not observed.

Eji Transition energy (in rydberg). The transition energy for the case of unobserved levels is obtained from the $f$ - and $S$-values as $E_{j i}=3 g i(f i j / S)$.

$g_{i}, g_{j} \quad$ The statistical weight factors of the initial and final states (levels).

$f_{i j} \quad$ Absorption oscillator strength (dimensionless).

$S \quad$ Line strength (in a.u).

$A_{j i} \quad$ Transition probability from upper state $j$ to lower state $i$ (in s ${ }^{-1}$ ). 


\begin{tabular}{|c|c|c|c|c|c|}
\hline \multicolumn{2}{|l|}{ Term } & \multirow{2}{*}{$\begin{array}{r}E(R y) \\
25.70221\end{array}$} & \multicolumn{2}{|l|}{ Term } & \multirow{2}{*}{$\begin{array}{r}E(R y) \\
4.43398\end{array}$} \\
\hline $3 s^{2} 3 p^{2}$ & $a^{1} S^{e}$ & & $3 p 3 d^{2} 4 p$ & $n^{3} S^{e}$ & \\
\hline $3 p^{4}$ & $b^{1} S^{e}$ & 20.33870 & $3 p 3 d^{2} 4 p$ & ${ }^{3} S^{e}$ & 4.02066 \\
\hline $3 s^{2} 3 d^{2}$ & $c{ }^{1} S^{e}$ & 18.57390 & $3 s^{2} 3 p\left({ }^{2} P^{o}\right) 7 p$ & $p^{3} S^{e}$ & 3.89122 \\
\hline $3 s 3 p^{2}\left({ }^{2} D^{e}\right) 3 d$ & $d^{1} S^{e}$ & 16.85690 & $3 s 3 p 3 d\left({ }^{2} F^{o}\right) 4 f$ & $q^{3} S^{e}$ & 3.84185 \\
\hline $3 s^{2} 3 p\left({ }^{2} P^{o}\right) 4 p$ & $e^{1} S^{e}$ & 12.54580 & $3 s^{2} 3 d\left({ }^{2} D^{e}\right) 5 d$ & $r^{3} S^{e}$ & 2.96766 \\
\hline $3 s^{2} 3 d^{2}$ & $f^{1} S^{e}$ & 12.53420 & $3 s^{2} 3 p\left({ }^{2} P^{o}\right) 8 p$ & $s^{3} S^{e}$ & 2.88868 \\
\hline $3 s^{2} 3 d^{2}$ & $g^{1} S^{e}$ & 11.77120 & $3 p^{3}\left({ }^{2} P^{o}\right) 5 p$ & $t^{3} S^{e}$ & 2.43403 \\
\hline $3 s 3 p^{2}\left({ }^{2} S^{e}\right) 4 s$ & $h^{1} S^{e}$ & 10.61790 & $3 s 3 p^{2}\left({ }^{2} S^{e}\right) 6 s$ & $u^{3} S^{e}$ & 2.31419 \\
\hline $3 s^{2} 3 d^{2}$ & $i^{1} S^{e}$ & 10.08850 & $3 s^{2} 3 p\left({ }^{2} P^{o}\right) 9 p$ & $v^{3} S^{e}$ & 2.27397 \\
\hline $3 s 3 p^{2}\left({ }^{2} D^{e}\right) 4 d$ & $j^{1} S^{e}$ & 8.72829 & $3 s 3 p^{2}\left({ }^{2} D^{e}\right) 6 d$ & $w^{3} S^{e}$ & 2.20068 \\
\hline $3 s^{2} 3 p\left({ }^{2} P^{o}\right) 5 p$ & $k^{1} S^{e}$ & 7.87144 & $3 s^{2} 3 p\left({ }^{2} P^{o}\right) 10 p$ & $x^{3} S^{e}$ & 1.82828 \\
\hline $3 p^{3}\left({ }^{2} P^{o}\right) 4 p$ & $l^{1} S^{e}$ & 6.90315 & $3 s 3 p 3 d\left({ }^{4} P^{o}\right) 5 p$ & $y^{3} S^{e}$ & 1.78467 \\
\hline $3 s^{2} 3 d\left({ }^{2} D^{e}\right) 4 d$ & $m^{1} S^{e}$ & 6.69289 & $3 s 3 p^{3}$ & $z^{3} S^{0}$ & 22.75013 \\
\hline $3 s^{2} 3 p\left({ }^{2} P^{o}\right) 6 p$ & $n^{1} S^{e}$ & 5.28141 & $3 p^{3}\left({ }^{2} D^{o}\right) 3 d$ & $y^{3} S^{o}$ & 16.84160 \\
\hline $3 s 3 p^{2}\left({ }^{2} S^{e}\right) 5 s$ & ${ }^{1} S^{e}$ & 5.12048 & $3 s 3 p^{3}$ & $x^{3} S^{o}$ & 15.70260 \\
\hline $3 s 3 p^{2}\left({ }^{2} D^{e}\right) 5 d$ & $p^{1} S^{e}$ & 4.49350 & $3 s 3 p 3 d^{2}$ & $w^{3} S^{o}$ & 13.65450 \\
\hline $3 s^{2} 3 d^{2}$ & $q^{1} S^{e}$ & 4.04825 & $3 s 3 p^{2}\left({ }^{4} P^{e}\right) 4 p$ & $v^{3} S^{o}$ & 11.37000 \\
\hline $3 s^{2} 3 p\left({ }^{2} P^{o}\right) 7 p$ & $r^{1} S^{e}$ & 3.84490 & $3 s 3 p^{2}\left({ }^{2} P^{e}\right) 4 p$ & $u^{3} S^{o}$ & 9.43493 \\
\hline $3 s 3 p 3 d\left({ }^{2} F^{o}\right) 4 f$ & $s^{1} S^{e}$ & 3.78293 & $3 p 3 d^{2} 4 s$ & $t^{3} S^{\circ}$ & 8.67974 \\
\hline $3 s 3 d^{2} 4 s$ & $t^{1} S^{e}$ & 3.61111 & $3 p^{3}\left({ }^{2} D^{o}\right) 4 d$ & $s^{3} S^{o}$ & 6.77336 \\
\hline $3 s^{2} 3 p\left({ }^{2} P^{o}\right) 8 p$ & $u^{1} S^{e}$ & 2.90651 & $3 s 3 p^{2}\left({ }^{4} P^{e}\right) 5 p$ & $r^{3} S^{o}$ & 5.96824 \\
\hline $3 s^{2} 3 d\left({ }^{2} D^{e}\right) 5 d$ & $v^{1} S^{e}$ & 2.71124 & $3 s 3 p 3 d\left({ }^{4} D^{o}\right) 4 d$ & $q^{3} S^{\circ}$ & 5.67155 \\
\hline $3 s 3 p^{2}\left({ }^{2} S^{e}\right) 6 s$ & $w^{1} S^{e}$ & 2.34167 & $3 s 3 p 3 d\left({ }^{2} D^{o}\right) 4 d$ & $p^{3} S^{o}$ & 5.27257 \\
\hline $3 s^{2} 3 p\left({ }^{2} P^{o}\right) 9 p$ & $x^{1} S^{e}$ & 2.25478 & $3 s 3 p^{2}\left({ }^{2} P^{e}\right) 5 p$ & ${ }^{3} S^{\circ}$ & 4.38684 \\
\hline $3 s 3 p^{2}\left({ }^{2} D^{e}\right) 6 d$ & $y^{1} S^{e}$ & 2.19419 & $3 s 3 p^{2}\left({ }^{4} P^{e}\right) 6 p$ & $n^{3} S^{\circ}$ & 3.34518 \\
\hline $3 p^{3}\left({ }^{2} P^{o}\right) 5 p$ & $z^{1} S^{e}$ & 2.15829 & $3 p 3 d^{2} 4 s$ & $m^{3} S^{o}$ & 3.11050 \\
\hline $3 s^{2} 3 p\left({ }^{2} P^{o}\right) 10 p$ & ${ }^{1} S^{e}$ & 1.81444 & $3 p^{3}\left({ }^{4} S^{\circ}\right) 4 s$ & $l^{3} S^{o}$ & 3.07606 \\
\hline $3 p^{3}\left({ }^{2} D^{\circ}\right) 3 d$ & $z^{1} S^{o}$ & 17.78060 & $3 p^{3}\left({ }^{2} D^{o}\right) 5 d$ & $k^{3} S^{\circ}$ & 2.38623 \\
\hline $3 s 3 p 3 d^{2}$ & $y^{1} S^{\circ}$ & 15.06390 & $3 s 3 d^{2} 4 p$ & $j^{3} S^{o}$ & 2.18344 \\
\hline $3 s 3 p^{2}\left({ }^{2} P^{e}\right) 4 p$ & $x^{1} S^{\circ}$ & 9.89850 & $3 s 3 p^{2}\left({ }^{4} P^{e}\right) 7 p$ & $i^{3} S^{o}$ & 1.79494 \\
\hline $3 p^{3}\left({ }^{2} D^{\circ}\right) 4 d$ & $w^{1} S^{\circ}$ & 6.74732 & $3 s 3 p^{2}\left({ }^{2} P^{e}\right) 6 p$ & $h^{3} S^{o}$ & 1.75493 \\
\hline $3 s 3 p 3 d\left({ }^{2} D^{\circ}\right) 4 d$ & $v^{1} S^{o}$ & 5.34194 & $3 s 3 d^{2} 4 p$ & $g^{3} S^{o}$ & 1.67206 \\
\hline $3 s 3 p^{2}\left({ }^{2} P^{e}\right) 5 p$ & $u^{1} S^{o}$ & 4.48586 & $3 d^{2} 4 s 4 p$ & $f^{3} S^{o}$ & 1.53061 \\
\hline $3 p 3 d^{2} 4 s$ & $t^{1} S^{o}$ & 2.53911 & $3 s 3 p 3 d\left({ }^{4} D^{o}\right) 5 d$ & $e^{3} S^{o}$ & 1.18345 \\
\hline $3 p^{3}\left({ }^{2} D^{0}\right) 5 d$ & $s^{1} S^{o}$ & 2.39754 & $3 s 3 p 3 d\left({ }^{2} D^{o}\right) 5 d$ & $d^{3} S^{o}$ & 0.91255 \\
\hline $3 s 3 d^{2} 4 p$ & $r^{1} S^{o}$ & 2.18391 & $3 s 3 p^{2}\left({ }^{4} P^{e}\right) 8 p$ & $c^{3} S^{o}$ & 0.81287 \\
\hline $3 s 3 p^{2}\left({ }^{2} P^{e}\right) 6 p$ & $q^{1} S^{o}$ & 1.82283 & $3 s 3 p^{2}\left({ }^{2} P^{e}\right) 7 p$ & $b^{3} S^{o}$ & 0.29547 \\
\hline $3 s 3 p 3 d\left({ }^{2} D^{o}\right) 5 d$ & $p^{1} S^{o}$ & 0.93241 & $3 p^{3}\left({ }^{4} S^{o}\right) 5 s$ & $a^{3} S^{o}$ & 0.25149 \\
\hline $3 s 3 p^{2}\left({ }^{2} P^{e}\right) 7 p$ & ${ }^{1} S^{o}$ & 0.31470 & $3 s 3 p^{2}\left({ }^{4} P^{e}\right) 9 p$ & ${ }^{3} S^{\circ}$ & 0.14484 \\
\hline $3 d^{2} 4 s 4 p$ & $n^{1} S^{\circ}$ & 0.24249 & $3 d^{2} 4 s 4 p$ & ${ }^{3} S^{\circ}$ & 0.09112 \\
\hline $3 p^{3}\left({ }^{2} D^{0}\right) 6 d$ & $m^{1} S^{o}$ & 0.08156 & $3 p^{3}\left({ }^{2} D^{o}\right) 6 d$ & ${ }^{3} S^{\circ}$ & 0.04805 \\
\hline $3 s 3 p^{2}\left({ }^{2} D^{e}\right) 3 d$ & $a^{3} S^{e}$ & 19.23670 & $3 p^{2} 3 d^{2}$ & $a^{5} S^{e}$ & 13.59540 \\
\hline $3 s^{2} 3 p\left(^{2} P^{o}\right) 4 p$ & $b^{3} S^{e}$ & 13.11400 & $3 s 3 p 3 d\left({ }^{4} P^{o}\right) 4 p$ & $b^{5} S^{e}$ & 7.04687 \\
\hline $3 p^{2} 3 d^{2}$ & $c^{3} S^{e}$ & 11.85170 & $3 s 3 p 3 d\left({ }^{4} F^{o}\right) 4 f$ & $c^{5} S^{e}$ & 5.02703 \\
\hline $3 s 3 p^{2}\left({ }^{2} S^{e}\right) 4 s$ & $d^{3} S^{e}$ & 10.92440 & $3 s 3 p 3 d\left({ }^{4} P^{o}\right) 5 p$ & $d^{5} S^{e}$ & 1.89379 \\
\hline $3 s 3 p^{2}\left({ }^{2} D^{e}\right) 4 d$ & $e^{3} S^{e}$ & 8.85900 & $3 s 3 p 3 d\left({ }^{4} F^{o}\right) 5 f$ & $e^{5} S^{e}$ & 1.20975 \\
\hline $3 s^{2} 3 p\left({ }^{2} P^{o}\right) 5 p$ & $f^{3} S^{e}$ & 7.98549 & $3 s 3 p^{2}$ & $z^{5} S^{o}$ & 24.73540 \\
\hline $3 p^{3}\left({ }^{2} P^{o}\right) 4 p$ & $g^{3} S^{e}$ & 7.48290 & $3 s 3 p 3 d^{2}$ & $y^{5} S^{\circ}$ & 15.74340 \\
\hline $3 s^{2} 3 d\left({ }^{2} D^{e}\right) 4 d$ & $h^{3} S^{e}$ & 7.34698 & $3 s 3 p^{2}\left({ }^{4} P^{e}\right) 4 p$ & $x^{5} S^{\circ}$ & 10.83650 \\
\hline $3 s 3 p 3 d\left({ }^{4} P^{o}\right) 4 p$ & $i^{3} S^{e}$ & 6.64896 & $3 p^{3}\left({ }^{4} S^{o}\right) 4 s$ & $w^{5} S^{o}$ & 8.99575 \\
\hline $3 s^{2} 3 p\left({ }^{2} P^{o}\right) 6 p$ & $j^{3} S^{e}$ & 5.37570 & $3 s 3 p^{2}\left({ }^{4} P^{e}\right) 5 p$ & $v^{5} S^{o}$ & 5.90001 \\
\hline $3 s 3 p^{2}\left({ }^{2} S^{e}\right) 5 s$ & $k^{3} S^{e}$ & 5.19605 & $3 s 3 p 3 d\left({ }^{4} D^{o}\right) 4 d$ & $u{ }^{5} S^{\circ}$ & 5.46442 \\
\hline $3 s 3 p 3 d\left({ }^{4} F^{o}\right) 4 f$ & $l^{3} S^{e}$ & 4.75150 & $3 s 3 p^{2}\left({ }^{4} P^{e}\right) 6 p$ & $t^{5} S^{\circ}$ & 3.28447 \\
\hline $3 s 3 p^{2}\left({ }^{2} D^{e}\right) 5 d$ & $m^{3} S^{e}$ & 4.52973 & $3 p^{3}\left({ }^{4} S^{o}\right) 5 s$ & $s^{5} S^{\circ}$ & 3.16295 \\
\hline
\end{tabular}

TABLE I. Bound States of Fe XIII 
See page 137 for Explanation of Tables

\begin{tabular}{|c|c|c|c|c|c|}
\hline \multicolumn{2}{|l|}{ Term } & \multirow{2}{*}{$\begin{array}{r}E(R y) \\
2.42299\end{array}$} & \multicolumn{2}{|l|}{ Term } & \multirow{2}{*}{$\begin{array}{r}E(R y) \\
8.34900\end{array}$} \\
\hline $3 p 3 d^{2} 4 s$ & $r^{5} S^{\circ}$ & & $3 s 3 p^{2}\left({ }^{2} D^{e}\right) 4 f$ & $l^{1} P^{o}$ & \\
\hline $3 s 3 p^{2}\left({ }^{4} P^{e}\right) 7 p$ & $q^{5} S^{\circ}$ & 1.75834 & $3 p 3 d^{2} 4 s$ & $k^{1} P^{o}$ & 7.79647 \\
\hline $3 s 3 d^{2} 4 p$ & $p^{5} S^{\circ}$ & 1.57516 & $3 s^{2} 3 p\left({ }^{2} P^{o}\right) 5 d$ & $j^{1} P^{o}$ & 7.26760 \\
\hline $3 s 3 p 3 d\left({ }^{4} D^{o}\right) 5 d$ & ${ }^{5} S^{\circ}$ & 1.04084 & $3 s^{2} 3 d\left({ }^{2} D^{e}\right) 4 f$ & $i^{1} P^{o}$ & 6.83244 \\
\hline $3 s 3 p^{2}\left({ }^{4} P^{e}\right) 8 p$ & $n^{5} S^{\circ}$ & 0.79347 & $3 p^{3}\left({ }^{2} D^{o}\right) 4 d$ & $h^{1} P^{o}$ & 6.15627 \\
\hline $3 p^{3}\left({ }^{4} S^{o}\right) 6 s$ & $m^{5} S^{o}$ & 0.29218 & $3 p^{3}\left({ }^{2} P^{o}\right) 4 d$ & $g^{1} P^{o}$ & 6.02721 \\
\hline $3 s 3 p^{2}\left({ }^{4} P^{e}\right) 9 p$ & $l^{5} S^{\circ}$ & 0.15202 & $3 s^{2} 3 p\left({ }^{2} P^{o}\right) 6 s$ & $f^{1} P^{o}$ & 5.62929 \\
\hline $3 s 3 p^{2}\left({ }^{2} D^{e}\right) 3 d$ & $a{ }^{1} P^{e}$ & 19.39130 & $3 s 3 p 3 d\left({ }^{2} D^{0}\right) 4 d$ & $e^{1} P^{o}$ & 5.36663 \\
\hline $3 s 3 p^{2}\left({ }^{2} P^{e}\right) 3 d$ & $b^{1} P^{e}$ & 18.34830 & $3 s 3 p^{2}\left({ }^{2} D^{e}\right) 5 p$ & $d^{1} P^{o}$ & 5.15265 \\
\hline $3 p^{2} 3 d^{2}$ & $c^{1} P^{e}$ & 13.34590 & $3 s^{2} 3 p\left({ }^{2} P^{o}\right) 6 d$ & $c{ }^{1} P^{o}$ & 4.98880 \\
\hline $3 s^{2} 3 p\left({ }^{2} P^{o}\right) 4 p$ & $d^{1} P^{e}$ & 12.72803 & $3 s 3 p 3 d\left({ }^{2} F^{o}\right) 4 d$ & $b^{1} P^{o}$ & 4.82643 \\
\hline $3 s 3 p^{2}\left({ }^{2} P^{e}\right) 4 s$ & $e^{1} P^{e}$ & 12.27910 & $3 s 3 p^{2}\left({ }^{2} S^{e}\right) 5 p$ & $a^{1} P^{o}$ & 4.63680 \\
\hline $3 p^{2} 3 d^{2}$ & $f^{1} P^{e}$ & 10.61200 & $3 s 3 p^{2}\left({ }^{2} P^{e}\right) 5 p$ & ${ }^{1} P^{o}$ & 4.29605 \\
\hline $3 s 3 p^{2}\left({ }^{2} D^{e}\right) 4 d$ & ${ }^{1} P^{e}$ & 8.95844 & $3 s 3 p^{2}\left({ }^{2} D^{e}\right) 5 f$ & ${ }^{1} P^{o}$ & 4.04827 \\
\hline $3 s 3 p^{2}\left({ }^{2} P^{e}\right) 4 d$ & ${ }_{h}^{y} P^{e}$ & 8.25976 & $3 s^{2} 3 p\left({ }^{2} P^{o}\right) 7 s$ & ${ }^{1} P^{o}$ & 4.01057 \\
\hline $3 p^{3}\left({ }^{2} D^{\circ}\right) 4 p$ & $i^{1} P^{e}$ & 8.14948 & $3 s^{2} 3 p\left({ }^{2} P^{o}\right) 7 d$ & ${ }^{1} P^{o}$ & 3.66348 \\
\hline $3 s^{2} 3 p\left({ }^{2} P^{o}\right) 5 p$ & $j^{1} P^{e}$ & 8.00676 & $3 s^{2} 3 d\left({ }^{2} D^{e}\right) 4 p$ & ${ }^{1} P^{o}$ & 3.54517 \\
\hline $3 p^{3}\left({ }^{2} P^{o}\right) 4 p$ & $k^{1} P^{e}$ & 7.47086 & $3 s^{2} 3 p\left({ }^{2} P^{o}\right) 8 s$ & ${ }^{1} P^{o}$ & 3.01377 \\
\hline $3 s^{2} 3 d\left({ }^{2} D^{e}\right) 4 d$ & $l^{1} p^{e}$ & 7.34192 & $3 p 3 d^{2} 4 s$ & ${ }^{1} P^{o}$ & 2.99130 \\
\hline $3 s 3 p 3 d\left({ }^{4} P^{o}\right) 4 p$ & $m^{1} P^{e}$ & 6.61103 & $3 p^{3}\left({ }^{2} P^{o}\right) 5 s$ & ${ }^{1} P^{\circ}$ & 2.84666 \\
\hline $3 p^{3}\left({ }^{2} D^{\circ}\right) 4 f$ & $n^{1} P^{e}$ & 5.89068 & $3 s^{2} 3 p\left({ }^{2} P^{o}\right) 8 d$ & ${ }^{1} P^{o}$ & 2.76561 \\
\hline $3 s^{2} 3 p\left({ }^{2} P^{o}\right) 6 p$ & ${ }^{1} P^{e}$ & 5.42480 & $3 s 3 p^{2}\left({ }^{2} D^{e}\right) 6 p$ & ${ }^{1} P^{o}$ & 2.59802 \\
\hline $3 s 3 p^{2}\left({ }^{2} P^{e}\right) 5 s$ & $p^{1} P^{e}$ & 4.90643 & $3 s^{2} 3 d\left({ }^{2} D^{e}\right) 5 f$ & ${ }^{1} P^{o}$ & 2.44571 \\
\hline $3 s 3 p^{2}\left({ }^{2} D^{e}\right) 5 d$ & $q^{1} p^{e}$ & 4.57765 & $3 p^{3}\left({ }^{2} D^{\circ}\right) 5 d$ & ${ }^{1} P^{\circ}$ & 2.43918 \\
\hline $3 s 3 d^{2} 4 s$ & $r^{1} P^{e}$ & 4.50682 & $3 s^{2} 3 p\left({ }^{2} P^{o}\right) 9 s$ & ${ }^{1} P^{o}$ & 2.34788 \\
\hline $3 p 3 d^{2} 4 p$ & $s^{1} P^{e}$ & 4.36147 & $3 s^{2} 3 p\left({ }^{2} P^{o}\right) 9 d$ & ${ }^{1} P^{o}$ & 2.19125 \\
\hline $3 s 3 p 3 d\left({ }^{2} D^{o}\right) 4 f$ & $t^{1} P^{e}$ & 4.24337 & $3 p 3 d^{2} 4 s$ & ${ }^{1} P^{o}$ & 2.17356 \\
\hline $3 s^{2} 3 p\left({ }^{2} P^{o}\right) 7 p$ & $u{ }^{1} P^{e}$ & 3.90643 & $3 s 3 p^{2}\left({ }^{2} S^{e}\right) 6 p$ & ${ }^{1} P^{o}$ & 2.12216 \\
\hline $3 s 3 p 3 d\left({ }^{2} F^{0}\right) 4 f$ & $v^{1} P^{e}$ & 3.87729 & $3 p 3 d^{2} 4 s$ & ${ }^{1} P^{\circ}$ & 2.05176 \\
\hline $3 p 3 d^{2} 4 d$ & $w^{1} p^{e}$ & 3.80934 & $3 p 3 d^{2} 4 s$ & ${ }^{1} P^{o}$ & 1.99619 \\
\hline $3 s 3 p^{2}\left({ }^{2} P^{e}\right) 5 d$ & $x^{1} P^{e}$ & 3.58056 & $3 s 3 p^{2}\left({ }^{2} D^{e}\right) 6 f$ & ${ }^{1} P^{o}$ & 1.97143 \\
\hline $3 p^{3}\left({ }^{2} D^{o}\right) 5 p$ & $y^{1} P^{e}$ & 3.08545 & $3 p^{3}\left({ }^{2} P^{o}\right) 5 d$ & ${ }^{1} P^{o}$ & 1.91872 \\
\hline $3 s^{2} 3 d\left({ }^{2} D^{e}\right) 5 d$ & $z^{1} P^{e}$ & 2.99770 & $3 s^{2} 3 p\left({ }^{2} P^{o}\right) 10 s$ & ${ }^{1} P^{o}$ & 1.88051 \\
\hline $3 s^{2} 3 p\left({ }^{2} P^{o}\right) 8 p$ & ${ }^{1} P^{e}$ & 2.92493 & $3 s^{2} 3 p\left({ }^{2} P^{o}\right) 10 d$ & ${ }^{1} P^{o}$ & 1.75774 \\
\hline $3 p^{3}\left({ }^{2} P^{o}\right) 5 p$ & ${ }^{1} P^{e}$ & 2.42658 & $3 s 3 d^{2} 4 p$ & ${ }^{1} P^{o}$ & 1.70330 \\
\hline $3 s^{2} 3 p\left({ }^{2} P^{o}\right) 9 p$ & ${ }^{1} P^{e}$ & 2.29339 & $3 s 3 p^{2}\left({ }^{2} P^{e}\right) 6 p$ & ${ }^{1} P^{o}$ & 1.69150 \\
\hline $3 s 3 p^{2}\left({ }^{2} D^{e}\right) 6 d$ & ${ }^{1} P^{e}$ & 2.22734 & $3 s^{2} 3 p^{2}$ & $a^{3} P^{e}$ & 26.41387 \\
\hline $3 s 3 p^{2}\left({ }^{2} P^{e}\right) 6 s$ & ${ }^{1} P^{e}$ & 2.00431 & $3 p^{4}$ & $b^{3} P^{e}$ & 21.22360 \\
\hline $3 p^{3}\left({ }^{2} D^{\circ}\right) 5 f$ & ${ }^{1} P^{e}$ & 1.98794 & $3 p^{2} 3 d^{2}$ & $c^{3} P^{e}$ & 20.11330 \\
\hline $3 s^{2} 3 p\left({ }^{2} P^{o}\right) 10 p$ & ${ }^{1} P^{e}$ & 1.83096 & $3 s 3 p^{2}\left({ }^{4} P^{e}\right) 3 d$ & $d^{3} P^{e}$ & 19.32360 \\
\hline $3 s 3 p^{3}$ & $z^{1} P^{o}$ & 22.54429 & $3 s 3 p^{2}\left({ }^{2} D^{e}\right) 3 d$ & $e^{3} P^{e}$ & 18.38530 \\
\hline $3 s^{2} 3 p\left({ }^{2} P^{o}\right) 3 d$ & $y^{1} P^{o}$ & 21.33558 & $3 s 3 p^{2}\left({ }^{2} P^{e}\right) 3 d$ & $f^{3} P^{e}$ & 17.43740 \\
\hline $3 p^{3}\left({ }^{2} D^{\circ}\right) 3 d$ & $x^{1} P^{o}$ & 16.82610 & $3 p^{2} 3 d^{2}$ & $g^{3} P^{e}$ & 13.69680 \\
\hline $3 p^{3}\left({ }^{2} P^{o}\right) 3 d$ & $w^{1} P^{o}$ & 15.42320 & $3 p^{2} 3 d^{2}$ & ${ }^{3} P^{e}$ & 13.04560 \\
\hline $3 s 3 p 3 d^{2}$ & $v^{1} P^{o}$ & 14.52490 & $3 s^{2} 3 p\left({ }^{2} P^{o}\right) 4 p$ & $i^{3} P^{e}$ & 12.98730 \\
\hline $3 s 3 p 3 d^{2}$ & $u{ }^{1} P^{o}$ & 14.27960 & $3 p^{2} 3 d^{2}$ & $j^{3} P^{e}$ & 12.60220 \\
\hline $3 s^{2} 3 p\left({ }^{2} P^{o}\right) 4 s$ & $t^{1} P^{o}$ & 14.12619 & $3 s 3 p^{2}\left({ }^{4} P^{e}\right) 4 s$ & $k^{3} P^{e}$ & 12.15600 \\
\hline $3 s 3 p 3 d^{2}$ & $s{ }^{1} P^{o}$ & 13.27370 & $3 p^{2} 3 d^{2}$ & $l^{3} P^{e}$ & 11.87810 \\
\hline $3 s^{2} 3 p\left({ }^{2} P^{o}\right) 4 d$ & $r^{1} P^{o}$ & 11.49454 & $3 p^{2} 3 d^{2}$ & $m^{3} P^{e}$ & 11.75940 \\
\hline $3 s 3 p^{2}\left({ }^{2} D^{e}\right) 4 p$ & $q^{1} P^{o}$ & 10.37260 & $3 s 3 d^{2} 4 s$ & $n^{3} P^{e}$ & 11.24910 \\
\hline $3 s 3 p^{2}\left({ }^{2} S^{e}\right) 4 p$ & $p^{1} P^{o}$ & 9.79033 & $3 s 3 p^{2}\left({ }^{2} P^{e}\right) 4 s$ & ${ }^{3} P^{e}$ & 10.64900 \\
\hline $3 s 3 p^{2}\left({ }^{2} P^{e}\right) 4 p$ & ${ }^{1} P^{o}$ & 9.18263 & $3 s 3 p^{2}\left({ }^{4} P^{e}\right) 4 d$ & $p^{3} P^{e}$ & 9.70739 \\
\hline $3 p^{3}\left({ }^{2} P^{o}\right) 4 s$ & $n^{1} P^{o}$ & 8.64730 & $3 s 3 p^{2}\left({ }^{2} D^{e}\right) 4 d$ & $q^{3} p^{e}$ & 8.90153 \\
\hline $3 s^{2} 3 p\left({ }^{2} P^{o}\right) 5 s$ & $m^{1} P^{o}$ & 8.39159 & $3 p^{3}\left({ }^{2} D^{\circ}\right) 4 p$ & $r^{3} p^{e}$ & 8.21813 \\
\hline
\end{tabular}

TABLE I. Bound States of Fe XIII 
See page 137 for Explanation of Tables

\begin{tabular}{|c|c|c|c|c|c|}
\hline \multicolumn{2}{|l|}{ Term } & $E(R y)$ & \multicolumn{2}{|l|}{ Term } & $E(R y)$ \\
\hline $3 s 3 p^{2}\left({ }^{2} P^{e}\right) 4 d$ & $s^{3} P^{e}$ & 8.00618 & $3 s^{2} 3 p\left({ }^{2} P^{o}\right) 5 s$ & $j^{3} P^{o}$ & 8.42172 \\
\hline $3 s^{2} 3 p\left({ }^{2} P^{o}\right) 5 p$ & $t^{3} P^{e}$ & 7.95721 & $3 s 3 p^{2}\left({ }^{2} D^{e}\right) 4 f$ & $i^{3} P^{o}$ & 7.93301 \\
\hline $3 p^{3}\left({ }^{4} S^{\circ}\right) 4 p$ & $u^{3} P^{e}$ & 7.54662 & $3 s 3 p 3 d\left({ }^{4} P^{o}\right) 4 s$ & $h^{3} P^{o}$ & 7.76536 \\
\hline $3 s^{2} 3 d\left({ }^{2} D^{e}\right) 4 d$ & $v^{3} P^{e}$ & 7.21945 & $3 s^{2} 3 p\left({ }^{2} P^{o}\right) 5 d$ & $g^{3} P^{o}$ & 7.29486 \\
\hline $3 p^{3}\left({ }^{2} P^{o}\right) 4 p$ & $w^{3} P^{e}$ & 7.19575 & $3 p^{3}\left({ }^{2} D^{o}\right) 4 d$ & $f^{3} P^{o}$ & 6.79978 \\
\hline $3 s 3 p 3 d\left({ }^{4} P^{o}\right) 4 p$ & $x^{3} P^{e}$ & 7.11450 & $3 s^{2} 3 d\left({ }^{2} D^{e}\right) 4 f$ & $e^{3} P^{o}$ & 6.33175 \\
\hline $3 s 3 p 3 d\left({ }^{4} D^{o}\right) 4 p$ & $y^{3} P^{e}$ & 6.66223 & $3 p^{3}\left({ }^{2} P^{o}\right) 4 d$ & $d^{3} P^{o}$ & 6.12079 \\
\hline $3 s 3 p 3 d\left({ }^{2} D^{o}\right) 4 p$ & $z^{3} P^{e}$ & 6.55247 & $3 s 3 p 3 d\left({ }^{4} F^{o}\right) 4 d$ & $c^{3} \mathrm{P}^{o}$ & 5.88442 \\
\hline $3 s 3 p^{2}\left({ }^{4} P^{e}\right) 5 s$ & ${ }^{3} \mathrm{Pe}^{e}$ & 6.22370 & $3 s 3 p^{2}\left({ }^{4} P^{e}\right) 5 p$ & $b^{3} P^{o}$ & 5.81796 \\
\hline $3 p^{3}\left({ }^{2} D^{\circ}\right) 4 f$ & ${ }^{3} P^{e}$ & 5.82144 & $3 s^{2} 3 p\left({ }^{2} P^{o}\right) 6 s$ & $a^{3} P^{o}$ & 5.64806 \\
\hline $3 s^{2} 3 p\left({ }^{2} P^{o}\right) 6 p$ & ${ }^{3} P^{e}$ & 5.37877 & $3 s 3 p 3 d\left({ }^{4} P^{o}\right) 4 d$ & ${ }^{3} P^{o}$ & 5.56767 \\
\hline $3 s 3 p^{2}\left({ }^{4} P^{e}\right) 5 d$ & ${ }^{3} P^{e}$ & 5.24060 & $3 s 3 p 3 d\left({ }^{4} D^{o}\right) 4 d$ & ${ }^{3} P^{o}$ & 5.42970 \\
\hline $3 s 3 p^{2}\left({ }^{2} P^{e}\right) 5 s$ & ${ }^{3} P^{e}$ & 4.93857 & $3 s 3 p 3 d\left({ }^{2} D^{o}\right) 4 d$ & ${ }^{3} P^{o}$ & 5.31590 \\
\hline $3 s 3 p 3 d\left({ }^{4} F^{o}\right) 4 f$ & ${ }^{3} P^{e}$ & 4.86444 & $3 s 3 p^{2}\left({ }^{2} D^{e}\right) 5 p$ & ${ }^{3} P^{o}$ & 5.18493 \\
\hline $3 s 3 p 3 d\left({ }^{4} D^{\circ}\right) 4 f$ & ${ }^{3} P^{e}$ & 4.59704 & $3 s^{2} 3 p\left({ }^{2} P^{o}\right) 6 d$ & ${ }^{3} P^{o}$ & 5.00811 \\
\hline $3 s 3 p^{2}\left({ }^{2} D^{e}\right) 5 d$ & ${ }^{3} P^{e}$ & 4.56406 & $3 s 3 p 3 d\left({ }^{2} F^{o}\right) 4 d$ & ${ }^{3} P^{o}$ & 4.80345 \\
\hline $3 p 3 d^{2} 4 p$ & ${ }^{3} P^{e}$ & 4.50115 & $3 s 3 p^{2}\left({ }^{2} S^{e}\right) 5 p$ & ${ }^{3} P^{o}$ & 4.65617 \\
\hline $3 s 3 p 3 d\left({ }^{2} D^{o}\right) 4 f$ & ${ }^{3} P^{e}$ & 4.39037 & $3 s 3 p^{2}\left({ }^{2} P^{e}\right) 5 p$ & ${ }^{3} P^{o}$ & 4.38487 \\
\hline $3 p 3 d^{2} 4 p$ & ${ }^{3} P^{e}$ & 4.15762 & $3 s 3 p^{2}\left({ }^{2} D^{e}\right) 5 f$ & ${ }^{3} P^{o}$ & 4.10217 \\
\hline $3 s 3 p 3 d\left({ }^{2} F^{o}\right) 4 f$ & ${ }^{3} P^{e}$ & 3.94124 & $3 s^{2} 3 p\left({ }^{2} P^{o}\right) 7 s$ & ${ }^{3} P^{o}$ & 4.02638 \\
\hline $3 p 3 d^{2} 4 p$ & ${ }^{3} P^{e}$ & 3.93098 & $3 s^{2} 3 p\left({ }^{2} P^{o}\right) 7 d$ & ${ }^{3} \mathrm{P}^{o}$ & 3.64166 \\
\hline $3 s^{2} 3 p\left({ }^{2} P^{o}\right) 7 p$ & ${ }^{3} P^{e}$ & 3.86284 & $3 s^{2} 3 d\left({ }^{2} D^{e}\right) 5 p$ & ${ }^{3} P^{o}$ & 3.61553 \\
\hline $3 s 3 p^{2}\left({ }^{2} P^{e}\right) 5 d$ & ${ }^{3} P^{e}$ & 3.68440 & $3 s 3 p^{2}\left({ }^{4} P^{e}\right) 6 p$ & ${ }^{3} P^{o}$ & 3.21024 \\
\hline $3 s 3 p^{2}\left({ }^{4} P^{e}\right) 6 s$ & ${ }^{3} P^{e}$ & 3.48804 & $3 p 3 d^{2} 4 s$ & ${ }^{3} \mathrm{P}^{o}$ & 3.10670 \\
\hline $3 p^{3}\left({ }^{2} D^{o}\right) 5 p$ & ${ }^{3} P^{e}$ & 3.03085 & $3 s^{2} 3 p\left({ }^{2} P^{o}\right) 8 s$ & ${ }^{3} P^{o}$ & 3.02136 \\
\hline $3 s^{2} 3 p\left({ }^{2} P^{o}\right) 8 p$ & ${ }^{3} P^{e}$ & 2.93892 & $3 p^{3}\left({ }^{2} P^{o}\right) 5 s$ & ${ }^{3} P^{o}$ & 2.89167 \\
\hline $3 s 3 p^{2}\left({ }^{4} P^{e}\right) 6 d$ & ${ }^{3} P^{e}$ & 2.90778 & $3 s^{2} 3 p\left({ }^{2} P^{o}\right) 8 d$ & ${ }^{3} P^{o}$ & 2.77339 \\
\hline $3 s^{2} 3 d\left({ }^{2} D^{e}\right) 5 d$ & ${ }^{3} P^{e}$ & 2.86825 & $3 p 3 d^{2} 4 s$ & ${ }^{3} P^{o}$ & 2.67737 \\
\hline $3 p^{3}\left({ }^{4} S^{\circ}\right) 5 p$ & ${ }^{3} P^{e}$ & 2.57080 & $3 s 3 p^{2}\left({ }^{2} D^{e}\right) 6 p$ & ${ }^{3} P^{o}$ & 2.60483 \\
\hline $3 p^{3}\left({ }^{2} P^{o}\right) 5 p$ & ${ }^{3} P^{e}$ & 2.39476 & $3 p 3 d^{2} 4 s$ & ${ }^{3} P^{o}$ & 2.53394 \\
\hline $3 s^{2} 3 p\left({ }^{2} P^{o}\right) 9 p$ & ${ }^{3} P^{e}$ & 2.28463 & $3 s^{2} 3 d\left(^{2} D^{e}\right) 5 f$ & ${ }^{3} \mathrm{P}^{o}$ & 2.52056 \\
\hline $3 s 3 p^{2}\left({ }^{2} D^{e}\right) 6 d$ & ${ }^{3} P^{e}$ & 2.24150 & $3 p^{3}\left({ }^{2} D^{o}\right) 5 d$ & ${ }^{3} \mathrm{P}^{o}$ & 2.42465 \\
\hline $3 s 3 p^{2}\left({ }^{2} P^{e}\right) 6 s$ & ${ }^{3} P^{e}$ & 2.02679 & $3 p 3 d^{2} 4 s$ & ${ }^{3} \mathrm{P}^{o}$ & 2.35750 \\
\hline $3 p^{3}\left({ }^{2} D^{\circ}\right) 5 f$ & ${ }^{3} P^{e}$ & 1.96731 & $3 s^{2} 3 p\left({ }^{2} P^{o}\right) 9 s$ & ${ }^{3} P^{o}$ & 2.34869 \\
\hline $3 s 3 p 3 d\left({ }^{4} P^{o}\right) 5 p$ & ${ }^{3} P^{e}$ & 1.95218 & $3 s 3 p 3 d\left({ }^{4} P^{o}\right) 5 s$ & ${ }^{3} P^{o}$ & 2.28173 \\
\hline $3 s 3 p^{2}\left({ }^{4} P^{e}\right) 7 s$ & ${ }^{3} P^{e}$ & 1.90604 & $3 s 3 d^{2} 4 p$ & ${ }^{3} \mathrm{P}^{o}$ & 2.22445 \\
\hline $3 s^{2} 3 p\left({ }^{2} p^{o}\right) 10 p$ & ${ }^{3} P^{e}$ & 1.82900 & $3 s^{2} 3 p\left({ }^{2} P^{o}\right) 9 d$ & ${ }^{3} P^{o}$ & 2.17575 \\
\hline $3 s 3 p 3 d\left({ }^{4} D^{\circ}\right) 5 p$ & ${ }^{3} P^{e}$ & 1.74657 & $3 s 3 p^{2}\left({ }^{2} S^{e}\right) 6 p$ & ${ }^{3} P^{o}$ & 2.08147 \\
\hline $3 s 3 p^{3}$ & $z^{3} P^{o}$ & 23.52853 & $3 s 3 p^{2}\left({ }^{2} D^{e}\right) 6 f$ & ${ }^{3} P^{o}$ & 2.01950 \\
\hline $3 s^{2} 3 p\left({ }^{2} p^{o}\right) 3 d$ & $y^{3} P^{o}$ & 22.06081 & $3 s 3 d^{2} 4 p$ & ${ }^{3} \mathrm{Po}^{\circ}$ & 1.98338 \\
\hline $3 p^{3}\left({ }^{\frac{1}{2}} D^{\circ}\right) 3 d$ & $x^{3} P^{o}$ & 16.81400 & $3 s^{2} 3 p\left({ }^{\frac{1}{2}} P^{o}\right) 10 s$ & ${ }^{3} P^{o}$ & 1.88559 \\
\hline $3 p^{3}\left({ }^{2} P^{o}\right) 3 d$ & $w^{3} P^{o}$ & 16.70060 & $3 s 3 p^{2}\left({ }^{2} P^{e}\right) 6 p$ & ${ }^{3} P^{o}$ & 1.82325 \\
\hline $3 s 3 p 3 d^{2}$ & $v^{3} P^{o}$ & 15.34900 & $3 s^{2} 3 p\left({ }^{2} P^{o}\right) 10 d$ & ${ }^{3} \mathrm{P}^{\circ}$ & 1.75920 \\
\hline $3 s 3 p 3 d^{2}$ & $u^{3} P^{o}$ & 15.11790 & $3 s 3 p^{2}\left({ }^{4} P^{e}\right) 7 p$ & ${ }^{3} P^{o}$ & 1.73101 \\
\hline $3 s 3 p 3 d^{2}$ & $t^{3} P^{a}$ & 14.80190 & $3 p^{3}\left({ }^{2} P^{o}\right) 5 d$ & ${ }^{3} P^{o}$ & 1.70376 \\
\hline $3 s^{2} 3 p\left({ }^{2} P^{o}\right) 4 s$ & $s{ }^{3} P^{o}$ & 14.25442 & $3 s 3 p 3 d\left({ }^{4} F^{o}\right) 5 d$ & ${ }^{3} P^{o}$ & 1.69206 \\
\hline $3 s 3 p 3 d^{2}$ & $r^{3} P^{o}$ & 14.12170 & $3 s 3 p^{2}\left({ }^{4} P^{e}\right) 3 d$ & $a^{5} P^{e}$ & 20.26880 \\
\hline $3 s^{2} 3 p\left({ }^{2} P^{o}\right) 4 d$ & $q^{3} P^{o}$ & 11.69680 & $3 p^{2} 3 d^{2}$ & $b^{5} P^{e}$ & 13.29970 \\
\hline $3 s 3 p^{2}\left({ }^{4} P^{e}\right) 4 p$ & $p^{3} p^{o}$ & 10.90630 & $3 s 3 p^{2}\left({ }^{4} P^{e}\right) 4 s$ & $c^{5} P^{e}$ & 12.25010 \\
\hline $3 s 3 p^{2}\left({ }^{2} D^{e}\right) 4 p$ & ${ }^{3} P^{o}$ & 10.25060 & $3 s 3 p^{2}\left({ }^{4} P^{e}\right) 4 d$ & $d^{5} P^{e}$ & 9.73160 \\
\hline $3 s 3 p^{2}\left({ }^{2} S^{e}\right) 4 p$ & $n^{3} P^{o}$ & 9.81929 & $3 p^{3}\left({ }^{4} S^{\circ}\right) 4 p$ & $e^{5} P^{e}$ & 7.94300 \\
\hline $3 s 3 p^{2}\left({ }^{2} P^{e}\right) 4 p$ & $m^{3} P^{o}$ & 9.57119 & $3 s 3 p 3 d\left({ }^{4} P^{o}\right) 4 p$ & $f^{5} P^{e}$ & 7.06997 \\
\hline $3 s^{2} 3 d\left(^{2} D^{e}\right) 4 p$ & $l^{3} P^{o}$ & 8.79162 & $3 s 3 p 3 d\left({ }^{4} D^{o}\right) 4 p$ & $g^{5} P^{e}$ & 6.81191 \\
\hline $3 p^{3}\left({ }^{2} P^{o}\right) 4 s$ & $k^{3} P^{o}$ & 8.51198 & $3 s 3 p^{2}\left({ }^{4} P^{e}\right) 5 s$ & $h^{5} P^{e}$ & 6.35690 \\
\hline
\end{tabular}

TABLE I. Bound States of Fe XIII See page 137 for Explanation of Tables 


\begin{tabular}{|c|c|c|c|c|c|}
\hline \multicolumn{2}{|l|}{ Term } & \multirow{2}{*}{$\begin{array}{r}E(R y) \\
5.25024\end{array}$} & \multicolumn{2}{|l|}{ Term } & \multirow{2}{*}{$\begin{array}{r}E(R y) \\
8.37860\end{array}$} \\
\hline $3 s 3 p^{2}\left({ }^{4} P^{e}\right) 5 d$ & $i^{5} P^{e}$ & & $3 s 3 p^{2}\left({ }^{2} S^{e}\right) 4 d$ & $r^{1} D^{e}$ & \\
\hline $3 s 3 p 3 d\left({ }^{4} F^{o}\right) 4 f$ & $j^{5} P^{e}$ & 5.06483 & $3 s 3 p^{2}\left({ }^{2} P^{e}\right) 4 d$ & $s^{1} D^{e}$ & 8.10345 \\
\hline $3 s 3 p 3 d\left({ }^{4} D^{o}\right) 4 f$ & $k^{5} P^{e}$ & 4.65208 & $3 s^{2} 3 p\left({ }^{2} P^{o}\right) 5 p$ & $t^{1} D^{e}$ & 7.87168 \\
\hline $3 s 3 p^{2}\left({ }^{4} P^{e}\right) 6 s$ & $l^{5} P^{e}$ & 3.53401 & $3 p^{3}\left({ }^{2} D^{\circ}\right) 4 p$ & $u^{1} D^{e}$ & 7.59135 \\
\hline $3 s 3 p^{2}\left({ }^{4} P^{e}\right) 6 d$ & $m^{5} P^{e}$ & 2.92873 & $3 p^{3}\left({ }^{2} P^{o}\right) 4 p$ & $v^{1} D^{e}$ & 7.23674 \\
\hline $3 p^{3}\left({ }^{4} S^{o}\right) 5 p$ & ${ }^{5} P^{e}$ & 2.65628 & $3 s^{2} 3 d\left({ }^{2} D^{e}\right) 4 d$ & $w^{1} D^{e}$ & 7.18763 \\
\hline $3 s 3 p^{2}\left({ }^{4} P^{e}\right) 7 s$ & ${ }^{5} P^{e}$ & 1.94556 & $3 s^{2} 3 p\left({ }^{2} P^{o}\right) 5 f$ & $x^{1} D^{e}$ & 6.83179 \\
\hline $3 s 3 p 3 d\left({ }^{4} P^{o}\right) 5 p$ & $p^{5} P^{e}$ & 1.91542 & $3 s 3 p 3 d\left({ }^{2} F^{o}\right) 4 p$ & $y^{1} D^{e}$ & 6.59886 \\
\hline $3 s 3 p 3 d\left({ }^{4} D^{\circ}\right) 5 p$ & $q^{5} p^{e}$ & 1.81331 & $3 s 3 p 3 d\left({ }^{2} D^{\circ}\right) 4 p$ & $z^{1} D^{e}$ & 6.14627 \\
\hline $3 s 3 p^{2}\left({ }^{4} P^{e}\right) 7 d$ & $r^{5} P^{e}$ & 1.53104 & $3 p^{3}\left({ }^{2} D^{o}\right) 4 f$ & ${ }^{1} D^{e}$ & 5.83975 \\
\hline $3 s 3 p 3 d\left({ }^{4} F^{o}\right) 5 f$ & $s^{5} P^{e}$ & 1.22601 & $3 s 3 p^{2}\left({ }^{2} D^{e}\right) 5 s$ & ${ }^{1} D^{e}$ & 5.66113 \\
\hline $3 s 3 p^{2}\left({ }^{4} P^{e}\right) 8 s$ & $t^{5} P^{e}$ & 0.90140 & $3 s^{2} 3 p\left({ }^{2} P^{o}\right) 6 p$ & ${ }^{1} D^{e}$ & 5.34202 \\
\hline $3 s 3 p 3 d\left({ }^{4} D^{o}\right) 5 f$ & $u^{5} p^{e}$ & 0.78227 & $3 p^{3}\left({ }^{2} P^{o}\right) 4 f$ & ${ }^{1} D^{e}$ & 5.00426 \\
\hline $3 s 3 p^{2}\left({ }^{4} P^{e}\right) 8 d$ & $v^{5} P^{e}$ & 0.65237 & $3 s^{2} 3 p\left({ }^{2} P^{o}\right) 6 f$ & ${ }^{1} D^{e}$ & 4.74473 \\
\hline $3 s 3 d^{2} 4 s$ & $w^{5} P^{e}$ & 0.24717 & $3 s 3 p^{2}\left({ }^{2} D^{e}\right) 5 d$ & ${ }^{1} D^{e}$ & 4.61774 \\
\hline $3 s 3 p^{2}\left({ }^{4} P^{e}\right) 9 s$ & $x^{5} P^{e}$ & 0.22515 & $3 s 3 p 3 d\left({ }^{2} F^{o}\right) 4 f$ & ${ }^{1} D^{e}$ & 4.40654 \\
\hline $3 s 3 p^{2}\left({ }^{4} P^{e}\right) 9 d$ & $y^{5} P^{e}$ & 0.05999 & $3 s 3 p 3 d\left({ }^{2} D^{\circ}\right) 4 f$ & ${ }^{1} D^{e}$ & 4.32953 \\
\hline $3 p^{3}\left({ }^{4} S^{o}\right) 6 p$ & ${ }^{5} P^{e}$ & 0.00607 & $3 s^{2} 3 d\left({ }^{2} D^{e}\right) 5 s$ & ${ }^{1} D^{e}$ & 4.26833 \\
\hline $3 s 3 p 3 d^{2}$ & $z^{5} P^{o}$ & 16.29840 & $3 s 3 d^{2} 4 s$ & ${ }^{1} D^{e}$ & 4.04947 \\
\hline $3 s 3 p^{2}\left({ }^{4} P^{e}\right) 4 p$ & $y^{5} P^{o}$ & 11.17410 & $3 s 3 p^{2}\left({ }^{2} S^{e}\right) 5 d$ & ${ }^{1} D^{e}$ & 3.98115 \\
\hline $3 s 3 p 3 d\left({ }^{4} P^{o}\right) 4 s$ & $x^{5} P^{o}$ & 8.08878 & $3 p 3 d^{2} 4 p$ & ${ }^{1} D^{e}$ & 3.94884 \\
\hline $3 s 3 p 3 d\left({ }^{4} F^{o}\right) 4 d$ & $w^{5} P^{o}$ & 5.99827 & $3 s 3 p^{2}\left({ }^{2} D^{e}\right) 5 g$ & ${ }^{1} D^{e}$ & 3.88870 \\
\hline $3 s 3 p^{2}\left({ }^{4} P^{e}\right) 5 p$ & $v^{5} P^{o}$ & 5.90570 & $3 p 3 d^{2} 4 p$ & ${ }^{1} D^{e}$ & 3.87503 \\
\hline $3 s 3 p 3 d\left({ }^{4} P^{o}\right) 4 d$ & $u^{5} P^{o}$ & 5.77123 & $3 s^{2} 3 p\left({ }^{2} P^{o}\right) 7 p$ & ${ }^{1} D^{e}$ & 3.85177 \\
\hline $3 s 3 p 3 d\left({ }^{4} D^{o}\right) 4 d$ & $t^{5} P^{o}$ & 5.45267 & $3 p 3 d^{2} 4 p$ & ${ }^{1} D^{e}$ & 3.74896 \\
\hline $3 s 3 p^{2}\left({ }^{4} P^{e}\right) 6 p$ & $s^{5} P^{o}$ & 3.25801 & $3 s^{2} 3 p\left({ }^{2} P^{o}\right) 7 f$ & ${ }^{1} D^{e}$ & 3.48249 \\
\hline $3 p 3 d^{2} 4 s$ & $r^{5} P^{o}$ & 2.95730 & $3 s 3 p^{2}\left({ }^{2} P^{e}\right) 5 d$ & ${ }^{1} D^{e}$ & 3.37759 \\
\hline $3 s 3 d^{2} 4 p$ & $q^{5} p^{o}$ & 2.60111 & $3 p^{3}\left({ }^{2} D^{o}\right) 5 p$ & ${ }^{1} D^{e}$ & 2.96443 \\
\hline $3 s 3 p 3 d\left({ }^{4} P^{o}\right) 5 s$ & $p^{5} P^{o}$ & 2.35350 & $3 s^{2} 3 p\left({ }^{2} P^{o}\right) 8 p$ & ${ }^{1} D^{e}$ & 2.92333 \\
\hline $3 s 3 p^{2}\left({ }^{4} P^{e}\right) 7 p$ & ${ }^{5} P^{o}$ & 1.75684 & $3 s^{2} 3 d\left({ }^{2} D^{e}\right) 5 d$ & ${ }^{1} D^{e}$ & 2.86838 \\
\hline $3 s 3 p 3 d\left({ }^{4} F^{o}\right) 5 d$ & $n^{5} P^{o}$ & 1.63073 & $3 s 3 p^{2}\left({ }^{2} D^{e}\right) 6 s$ & ${ }^{1} D^{e}$ & 2.83864 \\
\hline $3 d^{2} 4 s 4 p$ & $m^{5} P^{o}$ & 1.31513 & $3 s^{2} 3 p\left({ }^{2} P^{o}\right) 8 f$ & ${ }^{1} D^{e}$ & 2.66373 \\
\hline $3 s 3 p 3 d\left({ }^{4} P^{o}\right) 5 d$ & $l^{5} P^{o}$ & 1.25526 & $3 s^{2} 3 d\left({ }^{2} D^{e}\right) 5 g$ & ${ }^{1} D^{e}$ & 2.38957 \\
\hline $3 s 3 p 3 d\left({ }^{4} D^{\circ}\right) 5 d$ & $k^{5} P^{o}$ & 1.15292 & $3 p^{3}\left({ }^{2} P^{o}\right) 5 p$ & ${ }^{1} D^{e}$ & 2.35831 \\
\hline $3 s 3 p 3 d\left({ }^{4} F^{o}\right) 5 g$ & $j^{5} P^{o}$ & 1.07780 & $3 s^{2} 3 p\left({ }^{2} P^{o}\right) 9 p$ & ${ }^{1} D^{e}$ & 2.27332 \\
\hline $3 s 3 p^{2}\left({ }^{4} P^{e}\right) 8 p$ & $i^{5} P^{o}$ & 0.79282 & $3 s 3 p^{2}\left({ }^{2} D^{e}\right) 6 d$ & ${ }^{1} D^{e}$ & 2.26562 \\
\hline $3 s 3 p^{2}\left({ }^{4} P^{e}\right) 9 p$ & $h^{5} P^{o}$ & 0.15647 & $3 s^{2} 3 p\left({ }^{2} P^{o}\right) 9 f$ & ${ }^{1} D^{e}$ & 2.10390 \\
\hline $3 s 23 p^{2}$ & $a^{1} D^{e}$ & 26.09807 & $3 p^{3}\left({ }^{2} D^{o}\right) 5 f$ & ${ }^{1} D^{e}$ & 1.96862 \\
\hline $3 p^{4}$ & $b^{1} D^{e}$ & 21.08020 & $3 s 3 p^{2}\left({ }^{2} D^{e}\right) 6 g$ & ${ }^{1} D^{e}$ & 1.91910 \\
\hline $3 s 3 p^{2}\left({ }^{2} D^{e}\right) 3 d$ & $c^{1} D^{e}$ & 18.98450 & $3 s^{2} 3 p\left({ }^{2} P^{o}\right) 10 p$ & ${ }^{1} D^{e}$ & 1.82629 \\
\hline $3 s 3 p^{2}\left({ }^{2} S^{e}\right) 3 d$ & $d^{1} D^{e}$ & 18.66270 & $3 s^{2} 3 p\left({ }^{2} P^{o}\right) 10 f$ & ${ }^{1} D^{e}$ & 1.70222 \\
\hline $3 s 3 p^{2}\left({ }^{2} P^{e}\right) 3 d$ & $e^{1} D^{e}$ & 17.77830 & $3 s 3 p^{3}$ & $z^{1} D^{\circ}$ & 23.23430 \\
\hline $3 s^{2} 3 d^{2}$ & $f^{1} D^{e}$ & 17.44850 & $3 s^{2} 3 p\left({ }^{2} P^{o}\right) 3 d$ & $y^{1} D^{\circ}$ & 21.99006 \\
\hline $3 p^{2} 3 d^{2}$ & $g^{1} D^{e}$ & 13.58120 & $3 p^{3}\left({ }^{2} D^{o}\right) 3 d$ & $x^{1} D^{o}$ & 17.16500 \\
\hline $3 s^{2} 3 p\left({ }^{2} P^{o}\right) 4 p$ & $r^{1} D^{e}$ & 12.97544 & $3 p^{3}\left({ }^{2} P^{0}\right) 3 d$ & $w^{1} D^{o}$ & 16.11440 \\
\hline $3 p^{2} 3 d^{2}$ & $i^{1} D^{e}$ & 12.64770 & $3 s 3 p 3 d^{2}$ & $v^{1} D^{o}$ & 15.20320 \\
\hline $3 p^{2} 3 d^{2}$ & $j^{1} D^{e}$ & 12.05190 & $3 s 3 p 3 d^{2}$ & $u^{1} D^{o}$ & 14.31200 \\
\hline $3 p^{2} 3 d^{2}$ & $k^{1} D^{e}$ & 11.98520 & $3 s 3 p 3 d^{2}$ & $t^{1} D^{o}$ & 13.59580 \\
\hline $3 s 3 p^{2}\left({ }^{2} D^{e}\right) 4 s$ & $l^{1} D^{e}$ & 11.32900 & $3 s^{2} 3 p\left({ }^{2} P^{o}\right) 4 d$ & $s^{1} D^{o}$ & 11.87440 \\
\hline $3 p^{2} 3 d^{2}$ & $m^{1} D^{e}$ & 11.28040 & $3 s 3 p^{2}\left({ }^{2} D^{e}\right) 4 p$ & $r^{1} D^{o}$ & 10.56320 \\
\hline $3 s^{2} 3 p\left({ }^{2} P^{o}\right) 4 f$ & $n^{1} D^{e}$ & 10.67276 & $3 s 3 p^{2}\left({ }^{2} P^{e}\right) 4 p$ & $q^{1} D^{o}$ & 9.35663 \\
\hline $3 p^{2} 3 d^{2}$ & ${ }^{1} D^{e}$ & 10.62370 & $3 p^{3}\left({ }^{2} D^{o}\right) 4 s$ & $p^{1} D^{o}$ & 8.98673 \\
\hline $3 s^{2} 3 d\left({ }^{2} D^{e}\right) 4 s$ & $p^{1} D^{e}$ & 9.74273 & $3 s^{2} 3 d\left({ }^{2} D^{e}\right) 4 p$ & ${ }^{1} D^{o}$ & 8.86781 \\
\hline $3 s 3 p^{2}\left({ }^{2} D^{e}\right) 4 d$ & $q^{1} D^{e}$ & 9.01091 & $3 s 3 p^{2}\left({ }^{2} D^{e}\right) 4 f$ & $n^{1} D^{o}$ & 7.97334 \\
\hline
\end{tabular}

TABLE I. Bound States of Fe XIII

See page 137 for Explanation of Tables 


\begin{tabular}{|c|c|c|c|c|c|}
\hline Term & & $E(R y)$ & Term & & $E(R y)$ \\
\hline $3 s 3 p 3 d\left({ }^{2} D^{o}\right) 4 s$ & $m^{1} D^{\circ}$ & 7.54170 & $3 s^{2} 3 p\left({ }^{2} P^{o}\right) 5 f$ & $w^{3} D^{e}$ & 6.90461 \\
\hline $3 s^{2} 3 p\left({ }^{2} P^{o}\right) 5 d$ & $l^{1} D^{\circ}$ & 7.37343 & $3 s 3 p 3 d\left({ }^{4} P^{o}\right) 4 p$ & $x^{3} D^{e}$ & 6.88817 \\
\hline $3 s 3 p^{2}\left({ }^{2} P^{e}\right) 4 f$ & $k^{1} D^{o}$ & 7.22863 & $3 s 3 p 3 d\left({ }^{4} D^{o}\right) 4 p$ & $y^{3} D^{e}$ & 6.67474 \\
\hline $3 p^{3}\left({ }^{2} D^{\circ}\right) 4 d$ & $j^{1} D^{\circ}$ & 6.72606 & $3 s 3 p 3 d\left({ }^{2} D^{o}\right) 4 p$ & $z^{3} D^{e}$ & 6.53270 \\
\hline $3 s^{2} 3 d\left({ }^{2} D^{e}\right) 4 f$ & $i{ }^{1} D^{o}$ & 6.49538 & $3 s 3 p 3 d\left({ }^{2} F^{0}\right) 4 p$ & ${ }^{3} D^{e}$ & 6.26734 \\
\hline $3 p^{3}\left({ }^{2} P^{o}\right) 4 d$ & $h^{1} D^{\circ}$ & 6.13398 & $3 p^{3}\left({ }^{2} D^{o}\right) 4 f$ & ${ }^{3} D^{e}$ & 5.88064 \\
\hline $3 s 3 p 3 d\left({ }^{2} D^{o}\right) 4 d$ & $g^{1} D^{o}$ & 5.35921 & $3 s 3 p^{2}\left(^{2} D^{e}\right) 5 s$ & ${ }^{3} D^{e}$ & 5.73671 \\
\hline $3 s 3 p^{2}\left({ }^{2} D^{e}\right) 5 p$ & $f^{1} D^{\circ}$ & 5.26320 & $3 s^{2} 3 p\left({ }^{2} P^{o}\right) 6 p$ & ${ }^{3} D^{e}$ & 5.38836 \\
\hline $3 s^{2} 3 p\left({ }^{2} P^{o}\right) 6 d$ & $e^{1} D^{\circ}$ & 5.04594 & $3 p^{3}\left({ }^{2} P^{o}\right) 4 f$ & ${ }^{3} D^{e}$ & 5.25421 \\
\hline $3 s 3 p 3 d\left({ }^{2} F^{o}\right) 4 d$ & $d^{1} D^{\circ}$ & 4.93075 & $3 s 3 p^{2}\left({ }^{4} P^{e}\right) 5 d$ & ${ }^{3} D^{e}$ & 5.14982 \\
\hline $3 s 3 p^{2}\left({ }^{2} P^{e}\right) 5 p$ & $c{ }^{1} D^{o}$ & 4.35607 & $3 s 3 p 3 d\left({ }^{4} F^{0}\right) 4 f$ & ${ }^{3} D^{e}$ & 5.03815 \\
\hline $3 s 3 p^{2}\left({ }^{2} D^{e}\right) 5 f$ & $b^{1} D^{o}$ & 4.11721 & $3 s^{2} 3 p\left({ }^{2} P^{o}\right) 6 f$ & ${ }^{3} D^{e}$ & 4.78170 \\
\hline $\left.3 s^{2} 3 d d^{2} D^{e}\right) 5 p$ & $a{ }^{1} D^{\circ}$ & 3.69441 & $3 s 3 p^{2}\left({ }^{2} D^{e}\right) 5 d$ & ${ }^{3} D^{e}$ & 4.65663 \\
\hline $3 s^{2} 3 p\left({ }^{2} P^{o}\right) 7 d$ & ${ }^{1} D^{o}$ & 3.66131 & $3 s 3 p 3 d\left({ }^{4} P^{o}\right) 4 f$ & ${ }^{3} D^{e}$ & 4.61040 \\
\hline $3 p^{3}\left({ }^{\frac{1}{2}} D^{\circ}\right) 5 s$ & ${ }^{1} D^{o}$ & 3.50725 & $3 s 3 p 3 d\left({ }^{4} D^{o}\right) 4 f$ & ${ }^{3} D^{e}$ & 4.50295 \\
\hline $3 s 3 p^{2}\left({ }^{2} P^{e}\right) 5 f$ & ${ }^{1} D^{o}$ & 3.33646 & $3 s 3 d^{2} 4 s$ & ${ }^{3} D^{e}$ & 4.43272 \\
\hline $3 s 3 p 3 d^{2}$ & ${ }^{1} D^{o}$ & 3.28906 & $3 s 3 p 3 d\left(^{2} D^{o}\right) 4 f$ & ${ }^{3} D^{e}$ & 4.36180 \\
\hline $3 s^{2} 3 p\left(^{2} P^{o}\right) 8 d$ & ${ }^{1} D^{o}$ & 2.78469 & $3 s^{2} 3 d\left(^{2} D^{e}\right) 5 s$ & ${ }^{3} D^{e}$ & 4.35358 \\
\hline $3 s 3 p^{2}\left({ }^{2} D^{e}\right) 6 p$ & ${ }^{1} D^{o}$ & 2.67315 & $3 p 3 d^{2} 4 p$ & ${ }^{3} D^{e}$ & 4.20242 \\
\hline $3 s^{2} 3 d\left(^{2} D^{e}\right) 5 f$ & ${ }^{1} D^{o}$ & 2.58692 & $3 p 3 d^{2} 4 p$ & ${ }^{3} D^{e}$ & 4.15851 \\
\hline $3 p^{3}\left(^{2} D^{\circ}\right) 5 d$ & ${ }^{1} D^{\circ}$ & 2.51616 & $3 s 3 p 3 d\left({ }^{2} F^{\circ}\right) 4 f$ & ${ }^{3} D^{e}$ & 4.02439 \\
\hline $3 p 3 d^{2} 4 s$ & ${ }^{1} D^{o}$ & 2.44803 & $3 s 3 p^{2}\left({ }^{2} S^{e}\right) 5 d$ & ${ }^{3} D^{e}$ & 3.96043 \\
\hline $3 p 3 d^{2} 4 s$ & ${ }^{1} D^{o}$ & 2.30185 & $3 s 3 p^{2}\left({ }^{2} D^{e}\right) 5 g$ & ${ }^{3} D^{e}$ & 3.91911 \\
\hline $3 s^{2} 3 p\left({ }^{2} P^{o}\right) 9 d$ & ${ }^{1} D^{\circ}$ & 2.18616 & $3 s^{2} 3 p\left({ }^{2} P^{o}\right) 7 p$ & ${ }^{3} D^{e}$ & 3.88437 \\
\hline $3 p 3 d^{2} 4 s$ & ${ }^{1} D^{o}$ & 2.16471 & $3 s^{2} 3 d\left({ }^{2} D^{e}\right) 6 s$ & ${ }^{3} D^{e}$ & 3.82406 \\
\hline $3 s 3 p 3 d\left({ }^{2} D^{o}\right) 5 s$ & ${ }^{1} D^{o}$ & 2.07388 & $3 s 3 p^{2}\left({ }^{2} P^{e}\right) 5 d$ & ${ }^{3} D^{e}$ & 3.64685 \\
\hline $3 s 3 p^{2}\left(^{2} D^{e}\right) 6 f$ & ${ }^{1} D^{o}$ & 2.00965 & $3 s^{2} 3 p\left({ }^{2} P^{o}\right) 7 f$ & ${ }^{3} D^{e}$ & 3.50121 \\
\hline $3 p 3 d^{2} 4 s$ & ${ }^{1} D^{o}$ & 1.95240 & $3 p^{3}\left({ }^{2} D^{o}\right) 5 p$ & ${ }^{3} D^{e}$ & 3.08494 \\
\hline $3 p^{3}\left({ }^{2} D^{\circ}\right) 5 g$ & ${ }^{1} D^{\circ}$ & 1.85658 & $3 s^{2} 3 d\left({ }^{2} D^{e}\right) 5 d$ & ${ }^{3} D^{e}$ & 3.01591 \\
\hline $3 s^{2} 3 p\left(^{2} P^{o}\right) 10 d$ & ${ }^{1} D^{o}$ & 1.77487 & $\left.3 s^{2} 3 p{ }^{2} P^{o}\right) 8 p$ & ${ }^{3} D^{e}$ & 2.93094 \\
\hline $3 p^{3}\left(^{2} P^{o}\right) 5 d$ & ${ }^{1} D^{o}$ & 1.76534 & $3 s 3 p^{2}\left(^{2} D^{e}\right) 6 s$ & ${ }^{3} D^{e}$ & 2.89570 \\
\hline $3 s 3 p^{2}\left({ }^{2} P^{e}\right) 6 p$ & ${ }^{1} D^{\circ}$ & 1.70450 & $3 s 3 p^{2}\left({ }^{4} P^{e}\right) 6 d$ & ${ }^{3} D^{e}$ & 2.85683 \\
\hline $3 s 3 p^{2}\left({ }^{4} P^{e}\right) 3 d$ & $a^{3} D^{e}$ & 19.70060 & $3 s^{2} 3 p\left({ }^{2} P^{o}\right) 8 f$ & ${ }^{3} D^{e}$ & 2.67694 \\
\hline $3 s 3 p^{2}\left(^{2} D^{e}\right) 3 d$ & $b^{3} D^{e}$ & 19.43030 & $3 p^{3}\left(^{2} P^{o}\right) 5 p$ & ${ }^{3} D^{e}$ & 2.41194 \\
\hline $3 s 3 p^{2}\left({ }^{2} S^{e}\right) 3 d$ & $c^{3} D^{e}$ & 19.07530 & $3 s^{2} 3 d\left({ }^{2} D^{e}\right) 5 g$ & ${ }^{3} D^{e}$ & 2.40210 \\
\hline $3 s 3 p^{2}\left({ }^{2} P^{e}\right) 3 d$ & $d^{3} D^{e}$ & 18.68930 & $3 s 3 p^{2}\left({ }^{2} D^{e}\right) 6 d$ & ${ }^{3} D^{e}$ & 2.29310 \\
\hline $3 p^{2} 3 d^{2}$ & $e^{3} D^{e}$ & 13.59400 & $3 s^{2} 3 p\left({ }^{2} P^{o}\right) 9 p$ & ${ }^{3} D^{e}$ & 2.28632 \\
\hline $3 s^{2} 3 p\left({ }^{2} P^{o}\right) 4 p$ & $f^{3} D^{e}$ & 13.23980 & $3 s 3 p 3 d\left({ }^{4} F^{0}\right) 5 p$ & ${ }^{3} D^{e}$ & 2.24956 \\
\hline $3 p^{2} 3 d^{2}$ & $g^{3} D^{e}$ & 13.07750 & $3 s^{2} 3 p\left({ }^{2} P^{o}\right) 9 f$ & ${ }^{3} D^{e}$ & 2.11292 \\
\hline $3 p^{2} 3 d^{2}$ & $h^{3} D^{e}$ & 12.30190 & $3 p^{3}\left(^{2} D^{\circ}\right) 5 f$ & ${ }^{3} D^{e}$ & 1.98124 \\
\hline $3 p^{2} 3 d^{2}$ & $i^{3} D^{e}$ & 12.25130 & $3 s 3 p^{2}\left(^{2} D^{e} 6 q\right.$ & ${ }^{3} D^{e}$ & 1.93602 \\
\hline $3 p^{2} 3 d^{2}$ & $j^{3} D^{e}$ & 11.92150 & $3 s 3 p 3 d\left({ }^{4} P^{o}\right) 5 p$ & ${ }^{3} D^{e}$ & 1.85437 \\
\hline $3 s 3 p^{2}\left({ }^{2} D^{e}\right) 4 s$ & $k^{3} D^{e}$ & 11.57790 & $3 s^{2} 3 p\left(^{2} P^{o}\right) 10 p$ & ${ }^{3} D^{e}$ & 1.83385 \\
\hline $\left.3 s^{2} 3 p{ }^{2} P^{o}\right) 4 f$ & $l^{3} D^{e}$ & 10.79310 & $\left.3 s 3 p^{2}{ }^{2} S^{e}\right) 6 d$ & ${ }^{3} D^{e}$ & 1.78658 \\
\hline $3 s^{2} 3 d\left({ }^{2} D^{e}\right) 4 s$ & $m^{3} D^{e}$ & 9.84355 & $3 s^{2} 3 p\left(^{2} P^{o}\right) 10 f$ & ${ }^{3} D^{e}$ & 1.70817 \\
\hline $3 s 3 p^{2}\left({ }^{4} P^{e}\right) 4 d$ & $n^{3} D^{e}$ & 9.48529 & $3 s 3 p^{3}$ & $z^{3} D^{\circ}$ & 23.90564 \\
\hline $3 s 3 p^{2}\left({ }^{2} D^{e}\right) 4 d$ & ${ }^{3} D^{e}$ & 9.09897 & $3 s^{2} 3 p\left({ }^{2} P^{o}\right) 3 d$ & $y^{3} D^{0}$ & 21.90079 \\
\hline $3 s 3 p^{2}\left(^{2} S^{e}\right) 4 d$ & $p^{3} D^{e}$ & 8.40825 & $3 s 3 p 3 d^{2}$ & $x^{3} D^{0}$ & 17.61890 \\
\hline $3 p^{3}\left({ }^{2} D^{\circ}\right) 4 p$ & $q^{3} D^{e}$ & 8.20682 & $3 p^{3}\left({ }^{2} D^{\circ}\right) 3 d$ & $w^{3} D^{o}$ & 16.81620 \\
\hline $3 s 3 p^{2}\left({ }^{2} P^{e}\right) 4 d$ & $r^{3} D^{e}$ & 8.10692 & $3 p^{3}\left({ }^{4} S^{o}\right) 3 d$ & $v^{3} D^{o}$ & 16.13850 \\
\hline $\left.3 s^{2} 3 p v^{2} P^{o}\right) 5 p$ & $s^{3} D^{e}$ & 7.96023 & $3 p^{3}\left(^{2} P^{o}\right) 3 d$ & $u^{3} D^{o}$ & 15.91050 \\
\hline $3 p^{3}\left({ }^{2} P^{\circ}\right) 4 p$ & $t^{3} D^{e}$ & 7.50718 & $3 s 3 p 3 d^{2}$ & $t^{3} D^{\circ}$ & 15.17420 \\
\hline $3 s^{2} 3 d\left({ }^{2} D^{e}\right) 4 d$ & $u^{3} D^{e}$ & 7.42121 & $3 s 3 p 3 d^{2}$ & $s^{3} D^{o}$ & 14.95520 \\
\hline $3 s 3 p 3 d\left({ }^{4} F^{\circ}\right) 4 p$ & $v^{3} D^{e}$ & 7.23814 & $3 s 3 p 3 d^{2}$ & $r^{3} D^{o}$ & 14.34170 \\
\hline
\end{tabular}

TABLE I. Bound States of Fe XIII

See page 137 for Explanation of Tables 


\begin{tabular}{|c|c|c|c|c|c|}
\hline Term & & $E(R y)$ & Term & & $E(R y)$ \\
\hline $3 s 3 p 3 d^{2}$ & $q^{3} D^{o}$ & 14.14670 & $3 s^{2} 3 p\left({ }^{2} P^{o}\right) 10 d$ & ${ }^{3} D^{\circ}$ & 1.74917 \\
\hline $3 s^{2} 3 p\left({ }^{2} P^{o}\right) 4 d$ & $p^{3} D^{o}$ & 11.90721 & $3 s 3 p^{2}\left({ }^{4} P^{e}\right) 7 p$ & ${ }^{3} D^{\circ}$ & 1.72344 \\
\hline $3 s 3 p^{2}\left({ }^{4} P^{e}\right) 4 p$ & ${ }^{3} D^{\circ}$ & 11.05500 & $3 p^{3}\left({ }^{2} P^{o}\right) 5 d$ & ${ }^{3} D^{\circ}$ & 1.69191 \\
\hline $3 s 3 p^{2}\left({ }^{2} D^{e}\right) 4 p$ & $n^{3} D^{o}$ & 10.37970 & $3 s 3 p^{2}\left({ }^{4} P^{e}\right) 3 d$ & $a^{5} D^{e}$ & 20.72660 \\
\hline $3 s 3 p^{2}\left({ }^{2} P^{e}\right) 4 p$ & $m^{3} D^{o}$ & 9.68365 & $3 p^{2} 3 d^{2}$ & $b^{5} D^{e}$ & 13.75500 \\
\hline $3 p^{3}\left({ }^{2} D^{o}\right) 4 s$ & $l^{3} D^{o}$ & 9.13775 & $3 p^{2} 3 d^{2}$ & $c^{5} D^{e}$ & 13.25220 \\
\hline $3 s^{2} 3 d\left({ }^{2} D^{e}\right) 4 p$ & $k^{3} D^{o}$ & 8.87769 & $3 s 3 p^{2}\left({ }^{4} P^{e}\right) 4 d$ & $d^{5} D^{e}$ & 9.54655 \\
\hline $3 s 3 p^{2}\left({ }^{4} P^{e}\right) 4 f$ & $j^{3} D^{o}$ & 8.71376 & $3 s 3 p 3 d\left({ }^{4} F^{o}\right) 4 p$ & $e^{5} D^{e}$ & 7.31467 \\
\hline $3 s 3 p^{2}\left({ }^{2} D^{e}\right) 4 f$ & $i^{3} D^{0}$ & 8.04520 & $3 s 3 p 3 d\left({ }^{4} P^{o}\right) 4 p$ & $f^{5} D^{e}$ & 7.11014 \\
\hline $3 s 3 p 3 d\left({ }^{4} D^{o}\right) 4 s$ & $h^{3} D^{o}$ & 7.78243 & $3 s 3 p 3 d\left({ }^{4} D^{o}\right) 4 p$ & $g^{5} D^{e}$ & 6.88149 \\
\hline $3 s 3 p 3 d\left({ }^{2} D^{\circ}\right) 4 s$ & $g^{3} D^{\circ}$ & 7.71645 & $3 s 3 p^{2}\left({ }^{4} P^{e}\right) 5 d$ & $h^{5} D^{e}$ & 5.19060 \\
\hline $3 s^{2} 3 p\left({ }^{2} P^{o}\right) 5 d$ & $f^{3} D^{o}$ & 7.36864 & $3 s 3 p 3 d\left({ }^{4} F^{o}\right) 4 f$ & $i^{5} D^{e}$ & 5.12546 \\
\hline $3 s 3 p^{2}\left({ }^{2} P^{e}\right) 4 f$ & $e^{3} D^{o}$ & 7.22400 & $3 s 3 p 3 d\left({ }^{4} P^{o}\right) 4 f$ & $j^{5} D^{e}$ & 4.86740 \\
\hline $3 p^{3}\left({ }^{2} D^{o}\right) 4 d$ & $d^{3} D^{\circ}$ & 6.78897 & $3 s 3 p 3 d\left({ }^{4} D^{\circ}\right) 4 f$ & $k^{5} D^{e}$ & 4.66707 \\
\hline $3 p^{3}\left({ }^{4} S^{o}\right) 4 d$ & $c^{3} D^{o}$ & 6.37441 & $3 s 3 p^{2}\left({ }^{4} P^{e}\right) 6 d$ & $l^{5} D^{e}$ & 2.87797 \\
\hline $3 s^{2} 3 d\left({ }^{2} D^{e}\right) 4 f$ & $b^{3} D^{o}$ & 6.34851 & $3 s 3 p 3 d\left({ }^{4} F^{o}\right) 5 p$ & $m^{5} D^{e}$ & 2.30223 \\
\hline $3 p^{3}\left({ }^{2} P^{o}\right) 4 d$ & $a^{3} D^{o}$ & 6.15146 & $3 s 3 p 3 d\left({ }^{4} P^{o}\right) 5 p$ & $n^{5} D^{e}$ & 1.91996 \\
\hline $3 s 3 p 3 d\left({ }^{4} F^{o}\right) 4 d$ & ${ }^{3} D^{\circ}$ & 6.03381 & $3 s 3 p 3 d\left({ }^{4} D^{o}\right) 5 p$ & ${ }^{5} D^{e}$ & 1.85577 \\
\hline $3 s 3 p^{2}\left({ }^{4} P^{e}\right) 5 p$ & ${ }^{3} D^{\circ}$ & 5.84479 & $3 s 3 p^{2}\left({ }^{4} P^{e}\right) 7 d$ & $p^{5} D^{e}$ & 1.51643 \\
\hline $3 s 3 p 3 d\left({ }^{4} P^{o}\right) 4 d$ & ${ }^{3} D^{\circ}$ & 5.70862 & $3 s 3 p 3 d\left({ }^{4} F^{0}\right) 5 f$ & $q^{5} D^{e}$ & 1.25173 \\
\hline $3 s 3 p 3 d\left({ }^{4} D^{o}\right) 4 d$ & ${ }^{3} D^{\circ}$ & 5.48396 & $3 s 3 p 3 d\left({ }^{4} P^{o}\right) 5 f$ & $r^{5} D^{e}$ & 0.87475 \\
\hline $3 s 3 p 3 d\left({ }^{2} D^{\circ}\right) 4 d$ & ${ }^{3} D^{\circ}$ & 5.36004 & $3 s 3 p 3 d\left({ }^{4} D^{\circ}\right) 5 f$ & $s^{5} D^{e}$ & 0.79698 \\
\hline $3 s 3 p^{2}\left({ }^{2} D^{e}\right) 5 p$ & ${ }^{3} D^{o}$ & 5.22939 & $3 s 3 p^{2}\left({ }^{4} P^{e}\right) 8 d$ & $t^{5} D^{e}$ & 0.64408 \\
\hline $3 s^{2} 3 p\left({ }^{2} P^{o}\right) 6 d$ & ${ }^{3} D^{o}$ & 5.04658 & $3 p 3 d^{2} 4 p$ & $u^{5} D^{e}$ & 0.56220 \\
\hline $3 s 3 p 3 d\left({ }^{2} F^{o}\right) 4 d$ & ${ }^{3} D^{\circ}$ & 4.93682 & $3 s 3 p^{2}\left({ }^{4} P^{e}\right) 9 d$ & $v^{5} D^{e}$ & 0.04967 \\
\hline $3 s 3 p^{2}\left({ }^{4} P^{e}\right) 5 f$ & ${ }^{3} D^{\circ}$ & 4.79026 & $3 p^{3}\left({ }^{4} S^{o}\right) 3 d$ & $z^{5} D^{0}$ & 17.54770 \\
\hline $3 s 3 p^{2}\left({ }^{2} P^{e}\right) 5 p$ & ${ }^{3} D^{\circ}$ & 4.42021 & $3 s 3 p 3 d^{2}$ & $y^{5} D^{0}$ & 16.45810 \\
\hline $3 s 3 p^{2}\left({ }^{2} D^{e}\right) 5 f$ & ${ }^{3} D^{\circ}$ & 4.15232 & $3 s 3 p 3 d^{2}$ & $x^{5} D^{\circ}$ & 16.09170 \\
\hline $3 s^{2} 3 d\left({ }^{2} D^{e}\right) 5 p$ & ${ }^{3} D^{0}$ & 3.70290 & $3 s 3 p^{2}\left({ }^{4} P^{e}\right) 4 p$ & $w^{5} D^{o}$ & 11.27290 \\
\hline $3 s^{2} 3 p\left({ }^{2} P^{o}\right) 7 d$ & ${ }^{3} D^{\circ}$ & 3.63105 & $3 s 3 p^{2}\left({ }^{4} P^{e}\right) 4 f$ & $v^{5} D^{\circ}$ & 8.80722 \\
\hline $3 p^{3}\left({ }^{2} D^{o}\right) 5 s$ & ${ }^{3} D^{\circ}$ & 3.53902 & $3 s 3 p 3 d\left({ }^{4} D^{o}\right) 4 s$ & $u^{5} D^{o}$ & 8.04349 \\
\hline $3 p 3 d^{2} 4 s$ & ${ }^{3} D^{0}$ & 3.33873 & $3 p^{3}\left({ }^{4} S^{o}\right) 4 d$ & $t^{5} D^{o}$ & 6.39515 \\
\hline $3 s 3 p^{2}\left({ }^{2} P^{e}\right) 5 f$ & ${ }^{3} D^{\circ}$ & 3.32588 & $3 s 3 p 3 d\left({ }^{4} F^{o}\right) 4 d$ & $s^{5} D^{o}$ & 6.09623 \\
\hline $3 s 3 p^{2}\left({ }^{4} P^{e}\right) 6 p$ & ${ }^{3} D^{\circ}$ & 3.24138 & $3 s 3 p^{2}\left({ }^{4} P^{e}\right) 5 p$ & $r^{5} D^{o}$ & 5.91927 \\
\hline $3 p 3 d^{2} 4 s$ & ${ }^{3} D^{o}$ & 3.08515 & $3 s 3 p 3 d\left({ }^{4} P^{o}\right) 4 d$ & $q^{5} D^{o}$ & 5.58853 \\
\hline $3 p 3 d^{2} 4 s$ & ${ }^{3} D^{\circ}$ & 2.88337 & $3 s 3 p 3 d\left({ }^{4} D^{o}\right) 4 d$ & $p^{5} D^{\circ}$ & 5.50054 \\
\hline $3 s^{2} 3 p\left({ }^{2} P^{o}\right) 8 d$ & ${ }^{3} D^{0}$ & 2.78196 & $3 s 3 p^{2}\left({ }^{4} P^{e}\right) 5 f$ & ${ }^{5} D^{o}$ & 4.82998 \\
\hline $3 p 3 d^{2} 4 s$ & ${ }^{3} D^{\circ}$ & 2.68709 & $3 s 3 p^{2}\left({ }^{4} P^{e}\right) 6 p$ & ${ }^{5} D^{o}$ & 3.28185 \\
\hline $3 s 3 p^{2}\left({ }^{4} P^{e}\right) 6 f$ & ${ }^{3} D^{\circ}$ & 2.66256 & $3 p 3 d^{2} 4 s$ & $m^{5} D^{o}$ & 3.03839 \\
\hline $3 s 3 p^{2}\left({ }^{2} D^{e}\right) 6 p$ & ${ }^{3} D^{\circ}$ & 2.65033 & $3 p 3 d^{2} 4 s$ & $l^{5} D^{\circ}$ & 2.87658 \\
\hline $3 s^{2} 3 d\left({ }^{2} D^{e}\right) 5 f$ & ${ }^{3} D^{\circ}$ & 2.53745 & $3 s 3 p^{2}\left({ }^{4} P^{e}\right) 6 f$ & $k^{5} D^{o}$ & 2.68207 \\
\hline $3 s 3 p 3 d\left({ }^{4} D^{\circ}\right) 5 s$ & ${ }^{3} D^{\circ}$ & 2.41181 & $3 s 3 p 3 d\left({ }^{4} D^{o}\right) 5 s$ & $j^{5} D^{o}$ & 2.39459 \\
\hline $3 p^{3}\left({ }^{2} D^{o}\right) 5 d$ & ${ }^{3} D^{0}$ & 2.37271 & $3 s 3 d^{2} 4 p$ & $i^{5} D^{o}$ & 2.28561 \\
\hline $3 p 3 d^{2} 4 s$ & ${ }^{3} D^{\circ}$ & 2.29661 & $3 p^{3}\left({ }^{4} S^{o}\right) 5 d$ & $h^{5} D^{o}$ & 1.95853 \\
\hline $3 s 3 d^{2} 4 p$ & ${ }^{3} D^{\circ}$ & 2.22814 & $3 s 3 p^{2}\left({ }^{4} P^{e}\right) 7 p$ & $g^{5} D^{\circ}$ & 1.76465 \\
\hline $3 s^{2} 3 p\left({ }^{2} P^{o}\right) 9 d$ & ${ }^{3} D^{\circ}$ & 2.18178 & $3 s 3 p 3 d\left({ }^{4} F^{o}\right) 5 d$ & $f^{5} D^{o}$ & 1.72715 \\
\hline $3 s 3 d^{2} 4 p$ & ${ }^{3} D^{\circ}$ & 2.15447 & $3 s 3 d^{2} 4 p$ & $e^{5} D^{o}$ & 1.59234 \\
\hline $3 s 3 p 3 d\left({ }^{2} D^{\circ}\right) 5 s$ & ${ }^{3} D^{\circ}$ & 2.11224 & $3 s 3 p^{2}\left({ }^{4} P^{e}\right) 7 f$ & $d^{5} D^{\circ}$ & 1.39517 \\
\hline $3 s 3 p^{2}\left({ }^{2} D^{e}\right) 6 f$ & ${ }^{3} D^{0}$ & 2.03573 & $3 d^{2} 4 s 4 p$ & $c^{5} D^{o}$ & 1.38366 \\
\hline $3 s 3 d^{2} 4 p$ & ${ }^{3} D^{\circ}$ & 1.93371 & $3 s 3 p 3 d\left({ }^{4} P^{o}\right) 5 d$ & $b^{5} D^{o}$ & 1.20490 \\
\hline $3 p^{3}\left({ }^{4} S^{o}\right) 5 d$ & ${ }^{3} D^{\circ}$ & 1.90221 & $3 s 3 p 3 d\left({ }^{4} D^{o}\right) 5 d$ & $a^{5} D^{o}$ & 1.13600 \\
\hline $3 p^{3}\left({ }^{2} D^{o}\right) 5 g$ & ${ }^{3} D^{o}$ & 1.85486 & $3 s 3 p 3 d\left({ }^{4} F^{o}\right) 5 g$ & ${ }^{5} D^{\circ}$ & 1.10603 \\
\hline $3 s 3 p^{2}\left({ }^{2} P^{e}\right) 6 p$ & ${ }^{3} D^{\circ}$ & 1.80395 & $3 s 3 p^{2}\left({ }^{4} P^{e}\right) 8 p$ & ${ }^{5} D^{\circ}$ & 0.80195 \\
\hline $3 s 3 p 3 d\left({ }^{4} F^{o}\right) 5 d$ & ${ }^{3} D^{0}$ & 1.76360 & $3 s 3 p 3 d\left({ }^{4} D^{o}\right)^{1} 5 g$ & ${ }^{5} D^{\circ}$ & 0.66144 \\
\hline
\end{tabular}

TABLE I. Bound States of Fe XIII

See page 137 for Explanation of Tables 


\begin{tabular}{|c|c|c|c|c|c|}
\hline \multicolumn{2}{|l|}{ Term } & \multirow{2}{*}{$\begin{array}{r}E(R y) \\
0.56321\end{array}$} & \multicolumn{2}{|l|}{ Term } & \multirow{2}{*}{$\begin{array}{r}E(R y) \\
5.38928\end{array}$} \\
\hline $3 s 3 p^{2}\left({ }^{4} P^{e}\right) 8 f$ & ${ }^{5} D^{\circ}$ & & $3 s 3 p 3 d\left({ }^{2} D^{o}\right) 4 d$ & $h^{1} F^{o}$ & \\
\hline $3 s 3 p^{2}\left({ }^{4} P^{e}\right) 9 p$ & ${ }^{5} D^{\circ}$ & 0.16217 & $3 s 3 p^{2}\left({ }^{2} D^{e}\right) 5 p$ & $g^{1} F^{\circ}$ & 5.18034 \\
\hline $3 s 3 p^{2}\left({ }^{4} P^{e}\right) 3 d$ & $a^{1} F^{e}$ & 19.65860 & $3 s 3 p 3 d\left({ }^{2} F^{\circ}\right) 4 d$ & $f^{1} F^{0}$ & 5.05736 \\
\hline $3 s 3 p^{2}\left(^{2} P^{e}\right) 3 d$ & $b^{1} F^{e}$ & 18.11920 & $3 s^{2} 3 p\left({ }^{2} P^{o}\right) 6 d$ & $e^{1} F^{o}$ & 4.99414 \\
\hline $3 p^{2} 3 d^{2}$ & $c^{1} F^{e}$ & 12.84000 & $3 s^{2} 3 p\left({ }^{2} P^{o}\right) 6 g$ & $d{ }^{1} F^{o}$ & 4.68009 \\
\hline $3 p^{2} 3 d^{2}$ & $d^{1} F^{e}$ & 12.38930 & $3 s 3 p^{2}\left({ }^{2} D^{e}\right) 5 f$ & $c^{1} F^{o}$ & 4.16298 \\
\hline $3 p^{2} 3 d^{2}$ & $e^{1} F^{e}$ & 11.43910 & $3 s^{2} 3 p\left({ }^{2} P^{o}\right) 7 d$ & $b^{1} F^{o}$ & 3.69385 \\
\hline $3 s^{2} 3 p\left({ }^{2} P^{o}\right) 4 f$ & $f^{1} F^{e}$ & 10.97690 & $3 s 3 p^{2}\left({ }^{2} S^{e}\right) 5 f$ & $a{ }^{1} F^{o}$ & 3.56501 \\
\hline $3 s 3 p^{2}\left({ }^{4} P^{e}\right) 4 d$ & $g^{1} F^{e}$ & 9.14639 & $3 s^{2} 3 d\left({ }^{2} D^{e}\right) 5 p$ & ${ }^{1} F^{\circ}$ & 3.54845 \\
\hline $3 p^{3}\left({ }^{2} D^{o}\right) 4 p$ & $h^{1} F^{e}$ & 8.22428 & $3 s^{2} 3 p\left({ }^{2} P^{o}\right) 7 g$ & ${ }^{1} F^{\circ}$ & 3.44606 \\
\hline $3 s 3 p^{2}\left({ }^{2} P^{e}\right) 4 d$ & $i^{1} F^{e}$ & 7.94341 & $3 p 3 d^{2} 4 s$ & ${ }^{1} F^{o}$ & 3.27131 \\
\hline $3 s^{2} 3 d\left(^{2} D^{e}\right) 4 d$ & $j^{1} F^{e}$ & 7.56019 & $3 s 3 p^{2}\left({ }^{2} P^{e}\right) 5 f$ & ${ }^{1} F^{\circ}$ & 3.20068 \\
\hline $3 s^{2} 3 p\left(^{2} P^{o}\right) 5 f$ & $k^{1} F^{e}$ & 6.97708 & $3 s^{2} 3 p\left({ }^{2} P^{o}\right) 8 d$ & ${ }^{1} F^{o}$ & 2.76996 \\
\hline $3 s 3 p 3 d\left({ }^{2} D^{\circ}\right) 4 p$ & $l^{1} F^{e}$ & 6.72357 & $3 s 3 p^{2}\left({ }^{2} D^{e}\right) 6 p$ & ${ }^{1} F^{\circ}$ & 2.67052 \\
\hline $3 s 3 p 3 d\left({ }^{2} F^{o}\right) 4 p$ & $m^{1} F^{e}$ & 6.42546 & $3 p 3 d^{2} 4 s$ & ${ }^{1} F^{\circ}$ & 2.64539 \\
\hline $3 p^{3}\left({ }^{2} D^{o}\right) 4 f$ & $n^{1} F^{e}$ & 5.72414 & $3 s^{2} 3 p\left({ }^{2} P^{o}\right) 8 g$ & ${ }^{1} F^{o}$ & 2.64003 \\
\hline $3 p^{3}\left({ }^{2} P^{o}\right) 4 f$ & ${ }^{1} F^{e}$ & 5.24671 & $3 s^{2} 3 d\left({ }^{2} D^{e}\right) 5 f$ & ${ }^{1} F^{\circ}$ & 2.52205 \\
\hline $3 s^{2} 3 p\left({ }^{2} P^{o}\right) 6 f$ & $p^{1} F^{e}$ & 4.81725 & $3 p 3 d^{2} 4 s$ & ${ }^{1} F^{\circ}$ & 2.31610 \\
\hline $3 s 3 p^{2}\left({ }^{2} D^{c}\right) 5 d$ & $q^{1} F^{e}$ & 4.64923 & $3 p^{3}\left({ }^{2} D^{\circ}\right) 5 d$ & ${ }^{1} F^{0}$ & 2.29670 \\
\hline $3 s 3 d^{2} 4 s$ & $r^{1} F^{e}$ & 4.40081 & $3 s^{2} 3 p\left({ }^{2} P^{o}\right) 9 d$ & ${ }^{1} F^{\circ}$ & 2.17581 \\
\hline $3 s 3 p 3 d\left({ }^{2} D^{o}\right) 4 f$ & $s^{1} F^{e}$ & 4.37566 & $3 s^{2} 3 p\left({ }^{2} P^{o}\right) 9 g$ & ${ }^{1} F^{o}$ & 2.08243 \\
\hline $3 s 3 p 3 d\left(^{2} F^{o}\right) 4 f$ & $t^{1} F^{e}$ & 4.10868 & $3 s 3 p^{2}\left({ }^{2} D^{e}\right) 6 f$ & ${ }^{1} F^{\circ}$ & 2.03714 \\
\hline $3 p 3 d^{2} 4 p$ & $u^{1} F^{e}$ & 4.03506 & $3 s 3 p^{2}\left({ }^{2} D^{e}\right) 6 h$ & ${ }^{1} F^{o}$ & 1.95276 \\
\hline $3 s 3 p^{2}\left({ }^{2} D^{e}\right) 5 g$ & $v^{1} F^{e}$ & 3.92337 & $3 s 3 p 3 d\left({ }^{2} F^{\circ}\right) 5 s$ & ${ }^{1} F^{\circ}$ & 1.91807 \\
\hline $3 s 3 p^{2}\left({ }^{2} P^{e}\right) 5 d$ & $w^{1} F^{e}$ & 3.54548 & $3 p^{3}\left({ }^{2} D^{\circ}\right) 5 g$ & ${ }^{1} F^{\circ}$ & 1.84873 \\
\hline $3 s^{2} 3 p\left({ }^{2} P^{o}\right) 7 f$ & $x^{1} F^{e}$ & 3.50448 & $3 p^{3}\left({ }^{2} P^{o}\right) 5 d$ & ${ }^{1} F^{o}$ & 1.81718 \\
\hline $3 s 3 p^{2}\left({ }^{2} P^{e}\right) 5 q$ & $y^{1} F^{e}$ & 3.19556 & $3 s^{2} 3 p\left({ }^{2} P^{o}\right) 10 d$ & ${ }^{1} F^{\circ}$ & 1.75071 \\
\hline $3 p^{3}\left({ }^{2} D^{o}\right) 5 p$ & $z^{1} F^{e}$ & 3.06425 & $3 s 3 p^{2}\left({ }^{4} P^{e}\right) 3 d$ & $a^{3} F^{e}$ & 20.49370 \\
\hline $3 s^{2} 3 d\left({ }^{2} D^{e}\right) 5 d$ & ${ }^{1} F^{e}$ & 3.03121 & $3 s 3 p^{2}\left({ }^{2} D^{e}\right) 3 d$ & $b^{3} F^{e}$ & 19.45930 \\
\hline $\left.3 s^{2} 3 p{ }^{2} P^{o}\right) 8 f$ & ${ }^{1} F^{e}$ & 2.69068 & $3 s 3 p^{2}\left({ }^{2} P^{e}\right) 3 d$ & $c^{3} F^{e}$ & 19.03570 \\
\hline $3 s^{2} 3 d\left(^{2} D^{e}\right) 5 q$ & ${ }^{1} F^{e}$ & 2.45098 & $3 s^{2} 3 d^{2}$ & $d^{3} F^{e}$ & 17.81980 \\
\hline $3 s 3 p^{2}\left({ }^{2} D^{e}\right) 6 d$ & ${ }^{1} F^{e}$ & 2.30302 & $3 p^{2} 3 d^{2}$ & $e^{3} F^{e}$ & 13.68870 \\
\hline $\left.3 s^{2} 3 p{ }^{2} P^{o}\right) 9 f$ & ${ }^{1} F^{e}$ & 2.12184 & $3 p^{2} 3 d^{2}$ & $f^{3} F^{e}$ & 13.40800 \\
\hline $3 s 3 p^{2}\left({ }^{2} D^{e}\right) 6 g$ & ${ }^{1} F^{e}$ & 1.97372 & $3 p^{2} 3 d^{2}$ & $g^{3} F^{e}$ & 12.84200 \\
\hline $3 p^{3}\left({ }^{2} D^{\circ}\right) 5 f$ & ${ }^{1} F^{e}$ & 1.88994 & $3 p^{2} 3 d^{2}$ & $h^{3} F^{e}$ & 12.41720 \\
\hline $3 s^{2} 3 p\left(^{2} P^{o}\right) 10 f$ & ${ }^{1} F^{e}$ & 1.71598 & $3 p^{2} 3 d^{2}$ & $i^{3} F^{e}$ & 12.22430 \\
\hline $3 s^{2} 3 p\left(^{2} P^{o}\right) 3 d$ & $z^{1} F^{0}$ & 21.46152 & $3 p^{2} 3 d^{2}$ & $j^{3} F^{e}$ & 11.62970 \\
\hline $3 p^{3}\left({ }^{2} D^{o}\right) 3 d$ & $y^{1} F^{o}$ & 16.34310 & $3 s^{2} 3 p\left({ }^{2} P^{o}\right) 4 f$ & $k^{3} F^{e}$ & 10.66829 \\
\hline $3 p^{3}\left({ }^{2} P^{o}\right) 3 d$ & $x^{1} F^{o}$ & 16.22130 & $3 s 3 p^{2}\left({ }^{4} P^{e}\right) 4 d$ & $l^{3} F^{e}$ & 9.62352 \\
\hline $3 s 3 p 3 d^{2}$ & $w^{1} F^{o}$ & 15.31410 & $3 s 3 p^{2}\left(^{2} D^{e}\right) 4 d$ & $m^{3} F^{e}$ & 9.09772 \\
\hline $3 s 3 p 3 d^{2}$ & $v^{1} F^{o}$ & 14.13020 & $3 s 3 p^{2}\left({ }^{2} P^{e}\right) 4 d$ & $n^{3} F^{e}$ & 8.29615 \\
\hline $3 s 3 p 3 d^{2}$ & $u^{1} F^{o}$ & 13.77260 & $3 p^{3}\left({ }^{2} D^{\circ}\right) 4 p$ & $o^{3} F^{e}$ & 7.97935 \\
\hline $3 s^{2} 3 p\left(^{2} P^{o}\right) 4 d$ & $t^{1} F^{1}$ & 11.67652 & $3 s 3 p 3 d\left({ }^{4} F^{\circ}\right) 4 p$ & $p^{3} F^{e}$ & 7.48616 \\
\hline $3 s 3 p^{2}\left(^{2} D^{e}\right) 4 p$ & $s^{1} F^{o}$ & 10.48330 & $3 s^{2} 3 d\left(^{2} D^{e}\right) 4 d$ & $q^{3} F^{e}$ & 7.38146 \\
\hline $3 s^{2} 3 d\left(^{2} D^{e}\right) 4 p$ & $r^{1} F^{o}$ & 8.72465 & $3 s^{2} 3 p\left({ }^{2} P^{o}\right) 5 f$ & $r^{3} F^{e}$ & 6.95822 \\
\hline $3 s 3 p^{2}\left(^{2} D^{e}\right) 4 f$ & $q^{1} F^{o}$ & 8.07117 & $3 s 3 p 3 d\left({ }^{4} D^{o}\right) 4 p$ & $s^{3} F^{e}$ & 6.76607 \\
\hline $3 s 3 p 3 d\left({ }^{2} F^{o}\right) 4 s$ & $p^{1} F^{o}$ & 7.43020 & $3 s 3 p 3 d\left(^{2} D^{o}\right) 4 p$ & $t^{3} F^{e}$ & 6.67936 \\
\hline $3 s^{2} 3 p\left(^{2} P^{o}\right) 5 d$ & ${ }^{1} F^{\circ}$ & 7.33385 & $3 s 3 p 3 d\left(^{2} F^{\circ}\right) 4 p$ & $u^{3} F^{e}$ & 6.36615 \\
\hline $3 s 3 p^{2}\left({ }^{2} S^{e}\right) 4 f$ & $n{ }^{1} F^{o}$ & 7.29288 & $3 p^{3}\left({ }^{2} D^{\circ}\right) 4 f$ & $v^{3} F^{e}$ & 5.95086 \\
\hline $3 s 3 p^{2}\left({ }^{2} P^{e}\right) 4 f$ & $m^{1} F^{o}$ & 7.05261 & $3 p^{3}\left({ }^{4} S^{\circ}\right) 4 f$ & $w^{3} F^{e}$ & 5.40230 \\
\hline $3 p^{3}\left({ }^{2} D^{o}\right) 4 d$ & $l^{1} F^{o}$ & 6.73390 & $\left.3 p^{3}{ }^{2} P^{o}\right) 4 f$ & $x^{3} F^{e}$ & 5.24573 \\
\hline $3 s^{2} 3 p\left({ }^{2} P^{o}\right) 5 g$ & $k^{1} F^{o}$ & 6.65311 & $3 s 3 p^{2}\left({ }^{4} P^{e}\right) 5 d$ & $y^{3} F^{e}$ & 5.18848 \\
\hline $3 s^{2} 3 d\left(^{2} D^{e}\right) 4 f$ & $j^{1} F^{\circ}$ & 6.31803 & $3 s 3 p 3 d\left({ }^{4} F^{0}\right) 4 f$ & $z^{3} F^{e}$ & 5.10814 \\
\hline $3 p^{3}\left({ }^{2} P^{\circ}\right) 4 d$ & $i^{1} F^{0}$ & 6.09574 & $3 s^{2} 3 p\left({ }^{2} P^{o}\right) 6 f$ & ${ }^{3} F^{e}$ & 4.80669 \\
\hline
\end{tabular}

TABLE I. Bound States of Fe XIII

See page 137 for Explanation of Tables 


\begin{tabular}{|c|c|c|c|c|c|}
\hline Term & & $E(R y)$ & Term & & $E(R y)$ \\
\hline $3 s 3 p 3 d\left({ }^{4} P^{o}\right) 4 f$ & ${ }^{3} F^{e}$ & 4.78834 & $3 s 3 p^{2}\left({ }^{4} P^{e}\right) 5 f$ & ${ }^{3} F^{o}$ & 4.65925 \\
\hline $3 s 3 p^{2}\left({ }^{2} D^{e}\right) 5 d$ & ${ }^{3} F^{e}$ & 4.67276 & $3 s 3 p^{2}\left({ }^{2} D^{e}\right) 5 f$ & ${ }^{3} F^{\circ}$ & 4.21575 \\
\hline $3 s 3 p 3 d\left({ }^{4} D^{o}\right) 4 f$ & ${ }^{3} F^{e}$ & 4.60032 & $3 s^{2} 3 d\left({ }^{2} D^{e}\right) 5 p$ & ${ }^{3} F^{o}$ & 3.68188 \\
\hline $3 s 3 p^{2}\left({ }^{4} P^{e}\right) 5 g$ & ${ }^{3} F^{e}$ & 4.52284 & $3 s^{2} 3 p\left({ }^{2} P^{o}\right) 7 d$ & ${ }^{3} F^{o}$ & 3.64602 \\
\hline $3 s 3 p 3 d\left({ }^{2} D^{o}\right) 4 f$ & ${ }^{3} F^{e}$ & 4.41192 & $3 s 3 p^{2}\left({ }^{2} S^{e}\right) 5 f$ & ${ }^{3} F^{\circ}$ & 3.59999 \\
\hline $3 s 3 p 3 d\left({ }^{2} F^{o}\right) 4 f$ & ${ }^{3} F^{e}$ & 4.35532 & $3 s^{2} 3 p\left({ }^{2} P^{o}\right) 7 g$ & ${ }^{3} F^{o}$ & 3.45864 \\
\hline $3 s 3 d^{2} 4 s$ & ${ }^{3} F^{e}$ & 4.20702 & $3 p 3 d^{2} 4 s$ & ${ }^{3} F^{o}$ & 3.34457 \\
\hline $3 s 3 d^{2} 4 s$ & ${ }^{3} F^{e}$ & 4.13834 & $3 s 3 p^{2}\left({ }^{2} P^{e}\right) 5 f$ & ${ }^{3} F^{o}$ & 3.29370 \\
\hline $3 s 3 p^{2}\left({ }^{2} D^{e}\right) 5 g$ & ${ }^{3} F^{e}$ & 3.95527 & $3 s 3 p 3 d\left({ }^{4} F^{o}\right) 5 s$ & ${ }^{3} F^{o}$ & 2.87940 \\
\hline $3 s 3 p^{2}\left({ }^{2} P^{e}\right) 5 d$ & ${ }^{3} F^{e}$ & 3.71707 & $3 p 3 d^{2} 4 s$ & ${ }^{3} F^{o}$ & 2.80081 \\
\hline $3 s^{2} 3 p\left({ }^{2} P^{o}\right) 7 f$ & ${ }^{3} F^{e}$ & 3.51678 & $3 s^{2} 3 p\left({ }^{2} P^{o}\right) 8 d$ & ${ }^{3} F^{o}$ & 2.77504 \\
\hline $3 s 3 p^{2}\left({ }^{2} P^{e}\right) 5 g$ & ${ }^{3} F^{e}$ & 3.19863 & $3 p 3 d^{2} 4 s$ & ${ }^{3} F^{o}$ & 2.70309 \\
\hline $3 p^{3}\left({ }^{2} D^{\circ}\right) 5 p$ & ${ }^{3} F^{e}$ & 3.06681 & $3 s^{2} 3 p\left({ }^{2} P^{o}\right) 8 g$ & ${ }^{3} F^{o}$ & 2.65551 \\
\hline $3 s^{2} 3 d\left({ }^{2} D^{e}\right) 5 d$ & ${ }^{3} F^{e}$ & 2.96701 & $3 s 3 p^{2}\left({ }^{2} D^{e}\right) 6 p$ & ${ }^{3} F^{o}$ & 2.62412 \\
\hline $3 s 3 p^{2}\left({ }^{4} P^{e}\right) 6 d$ & ${ }^{3} F^{e}$ & 2.89190 & $3 s^{2} 3 d\left({ }^{2} D^{e}\right) 6 f$ & ${ }^{3} F^{o}$ & 2.61639 \\
\hline $3 s^{2} 3 p\left({ }^{2} P^{o}\right) 8 f$ & ${ }^{3} F^{e}$ & 2.68678 & $3 s 3 p^{2}\left({ }^{4} P^{e}\right) 6 f$ & ${ }^{3} F^{o}$ & 2.59553 \\
\hline $3 s 3 p^{2}\left({ }^{4} P^{e}\right) 6 g$ & ${ }^{3} F^{e}$ & 2.58813 & $3 p^{3}\left({ }^{2} D^{\circ}\right) 5 d$ & ${ }^{3} F^{o}$ & 2.40565 \\
\hline $3 s^{2} 3 d\left({ }^{2} D^{e}\right) 5 g$ & ${ }^{3} F^{e}$ & 2.44619 & $3 p 3 d^{2} 4 s$ & ${ }^{3} F^{o}$ & 2.35271 \\
\hline $3 s 3 p 3 d\left({ }^{4} F^{0}\right) 5 p$ & ${ }^{3} F^{e}$ & 2.39463 & $3 s 3 d^{2} 4 p 3 p 3$ & ${ }^{3} F^{o}$ & 2.30647 \\
\hline $3 s 3 p^{2}\left({ }^{2} D^{e}\right) 6 d$ & ${ }^{3} F^{e}$ & 2.29745 & $3 s^{2} 3 p\left({ }^{2} P^{o}\right) 9 d$ & ${ }^{3} F^{o}$ & 2.17767 \\
\hline $3 s^{2} 3 p\left({ }^{2} P^{o}\right) 9 f$ & ${ }^{3} F^{e}$ & 2.11925 & $3 s^{2} 3 p\left({ }^{2} P^{o}\right) 9 g$ & ${ }^{3} F^{o}$ & 2.08767 \\
\hline $3 p^{3}\left({ }^{2} D^{o}\right) 5 f$ & ${ }^{3} F^{e}$ & 2.01162 & $3 s 3 p^{2}\left({ }^{2} D^{e}\right) 6 f$ & ${ }^{3} F^{o}$ & 2.06810 \\
\hline $3 s 3 p^{2}\left({ }^{2} D^{e}\right) 6 g$ & ${ }^{3} F^{e}$ & 1.95718 & $3 p^{3}\left({ }^{2} P^{o}\right) 5 d$ & ${ }^{3} F^{o}$ & 2.05439 \\
\hline $3 s 3 p 3 d\left({ }^{4} D^{o}\right) 5 p$ & ${ }^{3} F^{e}$ & 1.82815 & $3 s 3 p 3 d\left({ }^{2} F^{o}\right) 5 s$ & ${ }^{3} F^{o}$ & 2.02846 \\
\hline $3 s^{2} 3 p\left({ }^{2} P^{o}\right) 10 f$ & ${ }^{3} F^{e}$ & 1.71368 & $3 s 3 p^{2}\left({ }^{2} D^{e}\right) 6 h$ & ${ }^{3} F^{o}$ & 1.95243 \\
\hline $3 s^{2} 3 p\left({ }^{2} P^{o}\right) 3 d$ & $z^{3} F^{o}$ & 22.56580 & $3 s 3 d^{2} 4 p$ & ${ }^{3} F^{o}$ & 1.89710 \\
\hline $3 p^{3}\left({ }^{2} D^{\circ}\right) 3 d$ & $y^{3} F^{o}$ & 17.65550 & $3 p^{3}\left({ }^{2} D^{o}\right) 5 g$ & ${ }^{3} F^{o}$ & 1.85249 \\
\hline $3 p^{3}\left({ }^{2} P^{o}\right) 3 d$ & $x^{3} F^{o}$ & 16.71850 & $3 s^{2} 3 p\left({ }^{2} P^{o}\right) 10 d$ & ${ }^{3} F^{o}$ & 1.75752 \\
\hline $3 s 3 p 3 d^{2}$ & $w^{3} F^{0}$ & 16.08660 & $3 s^{2} 3 p\left({ }^{2} P^{o}\right) 10 g$ & ${ }^{3} F^{o}$ & 1.69336 \\
\hline $3 s 3 p 3 d^{2}$ & $v^{3} F^{o}$ & 15.39550 & $3 s 3 p^{2}\left({ }^{4} P^{e}\right) 3 d$ & $a^{5} F^{e}$ & 20.86860 \\
\hline $3 s 3 p 3 d^{2}$ & $u^{3} F^{o}$ & 15.06640 & $3 p^{2} 3 d^{2}$ & $b^{5} F^{e}$ & 13.77510 \\
\hline $3 s 3 p 3 d^{2}$ & $t^{3} F^{o}$ & 14.57740 & $3 s 3 p^{2}\left({ }^{4} P^{e}\right) 4 d$ & $c^{5} F^{e}$ & 9.66792 \\
\hline $3 s^{2} 3 p\left({ }^{2} P^{o}\right) 4 d$ & $s^{3} F^{o *}$ & 11.77721 & $3 s 3 p 3 d\left({ }^{4} F^{o}\right) 4 p$ & $d^{5} F^{e}$ & 7.44495 \\
\hline $3 s 3 p^{2}\left({ }^{2} D^{e}\right) 4 p$ & $r^{3} F^{o}$ & 10.57410 & $3 s 3 p 3 d\left({ }^{4} D^{o}\right) 4 p$ & $e^{5} F^{e}$ & 6.94902 \\
\hline $3 s 3 p^{2}\left({ }^{4} P^{e}\right) 4 f$ & $q^{3} F^{o}$ & 8.86677 & $3 p^{3}\left({ }^{4} S^{0}\right) 4 f$ & $f^{5} F^{e}$ & 5.61422 \\
\hline $3 s^{2} 3 d\left({ }^{2} D^{e}\right) 4 p$ & $p^{3} F^{o}$ & 8.42911 & $3 s 3 p 3 d\left({ }^{4} F^{o}\right) 4 f$ & $g^{5} F^{e}$ & 5.29792 \\
\hline $3 s 3 p 3 d\left({ }^{4} F^{0}\right) 4 s$ & $o^{3} F^{\circ}$ & 8.21781 & $3 s 3 p^{2}\left({ }^{4} P^{e}\right) 5 d$ & $h^{5} F^{e}$ & 5.22041 \\
\hline $3 s 3 p^{2}\left({ }^{2} D^{e}\right) 4 f$ & $n^{3} F^{o}$ & 8.19751 & $3 s 3 p 3 d\left({ }^{4} P^{o}\right) 4 f$ & $i^{5} F^{e}$ & 4.84926 \\
\hline $3 s 3 p^{2}\left({ }^{2} S^{e}\right) 4 f$ & $m^{3} F^{o}$ & 7.52718 & $3 s 3 p 3 d\left({ }^{4} D^{\circ}\right) 4 f$ & $j^{5} F^{e}$ & 4.71487 \\
\hline $3 s 3 p 3 d\left({ }^{2} F^{o}\right) 4 s$ & $l^{3} F^{o}$ & 7.42196 & $3 s 3 p^{2}\left({ }^{4} P^{e}\right) 5 g$ & $k^{5} F^{e}$ & 4.54236 \\
\hline $3 s^{2} 3 p\left({ }^{2} P^{o}\right) 5 d$ & $k^{3} F^{o}$ & 7.36212 & $3 s 3 p^{2}\left({ }^{4} P^{e}\right) 6 d$ & $l^{5} F^{e}$ & 2.90611 \\
\hline $3 s 3 p^{2}\left({ }^{2} P^{e}\right) 4 f$ & $j^{3} F^{o}$ & 7.21785 & $3 s 3 p^{2}\left({ }^{4} P^{e}\right) 6 g$ & $m^{5} F^{e}$ & 2.59601 \\
\hline $3 p^{3}\left({ }^{2} D^{\circ}\right) 4 d$ & $i^{3} F^{o}$ & 6.80941 & $3 s 3 p 3 d\left({ }^{4} F^{o}\right) 5 p$ & $n^{5} F^{e}$ & 2.37188 \\
\hline $3 s^{2} 3 p\left({ }^{2} P^{o}\right) 5 g$ & $h^{3} F^{o}$ & 6.71994 & $3 s 3 p 3 d\left({ }^{4} D^{o}\right) 5 p$ & $o^{5} F^{e}$ & 1.87854 \\
\hline $3 s^{2} 3 d\left({ }^{2} D^{e}\right) 4 f$ & $g^{3} F^{o}$ & 6.55566 & $3 p^{3}\left({ }^{4} S^{\circ}\right) 5 f$ & $p^{5} F^{e}$ & 1.62437 \\
\hline $3 p^{3}\left({ }^{2} P^{o}\right) 4 d$ & $f^{3} F^{o}$ & 6.13286 & $3 s 3 p^{2}\left({ }^{4} P^{e}\right) 7 d$ & $q^{5} F^{e}$ & 1.52349 \\
\hline $3 s 3 p 3 d\left({ }^{4} F^{o}\right) 4 d$ & $e^{3} F^{o}$ & 6.10406 & $3 s 3 p^{2}\left({ }^{4} P^{e}\right) 7 g$ & $r^{5} F^{e}$ & 1.33692 \\
\hline $3 s 3 p 3 d\left({ }^{4} P^{o}\right) 4 d$ & $d^{3} F^{o}$ & 5.60905 & $3 s 3 p 3 d\left({ }^{4} F^{o}\right) 5 f$ & $s^{5} F^{e}$ & 1.30899 \\
\hline $3 s 3 p 3 d\left({ }^{4} D^{o}\right) 4 d$ & $c^{3} F^{o}$ & 5.43327 & $3 s 3 p 3 d\left({ }^{4} P^{o}\right) 5 f$ & $t^{5} F^{e}$ & 0.86765 \\
\hline $3 s 3 p 3 d\left({ }^{2} D^{o}\right) 4 d$ & $b^{3} F^{o}$ & 5.36782 & $3 s 3 p 3 d\left({ }^{4} D^{o}\right) 5 f$ & $u^{5} F^{e}$ & 0.78293 \\
\hline $3 s 3 p^{2}\left({ }^{2} D^{e}\right) 5 p$ & $a^{3} F^{o}$ & 5.23830 & $3 p 3 d^{2} 4 p$ & $v^{5} F^{e}$ & 0.74646 \\
\hline $3 s 3 p 3 d\left({ }^{2} F^{0}\right) 4 d$ & ${ }^{3} F^{0}$ & 5.08009 & $3 s 3 p^{2}\left({ }^{4} P^{e}\right) 8 d$ & $w^{5} F^{e}$ & 0.64571 \\
\hline $3 s^{2} 3 p\left({ }^{2} P^{o}\right) 6 d$ & ${ }^{3} F^{\circ}$ & 4.99297 & $3 s 3 p^{2}\left({ }^{4} P^{e}\right) 8 g$ & $x^{5} F^{e}$ & 0.52287 \\
\hline $3 s^{2} 3 p\left({ }^{2} P^{o}\right) 6 g$ & ${ }^{3} F^{\circ}$ & 4.69622 & $3 s 3 p^{2}\left({ }^{4} P^{e}\right) 9 d$ & $y^{5} F^{e}$ & 0.05720 \\
\hline
\end{tabular}

TABLE I. Bound States of Fe XIII

See page 137 for Explanation of Tables 


\begin{tabular}{|c|c|c|c|c|c|}
\hline \multicolumn{2}{|l|}{ Term } & \multirow{2}{*}{$\begin{array}{r}E(R y) \\
16.39390\end{array}$} & \multicolumn{2}{|l|}{ Term } & \multirow{2}{*}{$\begin{array}{r}E(R y) \\
1.69038\end{array}$} \\
\hline $3 s 3 p 3 d^{2}$ & $z^{5} F^{o}$ & & $3 s^{2} 3 p\left({ }^{2} P^{o}\right) 10 h$ & $I^{1} G^{e}$ & \\
\hline $3 s 3 p^{2}\left({ }^{4} P^{e}\right) 4 f$ & $y^{5} F^{0}$ & 8.74722 & $3 p^{3}\left({ }^{2} D^{o}\right) 3 d$ & $z^{1} G^{o}$ & 17.07500 \\
\hline $3 s 3 p 3 d\left({ }^{4} F^{o}\right) 4 s$ & $x^{5} F^{o}$ & 8.51606 & $3 s 3 p 3 d^{2}$ & $y^{1} G^{o}$ & 15.26960 \\
\hline $3 s 3 p 3 d\left(^{4} F^{o}\right) 4 d$ & $w^{5} F^{o}$ & 6.22411 & $3 s 3 p 3 d^{2}$ & $x^{1} G^{o}$ & 14.04160 \\
\hline $3 s 3 p 3 d\left({ }^{4} P^{o}\right) 4 d$ & $v^{5} F^{\circ}$ & 5.71943 & $3 s 3 p^{2}\left({ }^{2} D^{e}\right) 4 f$ & $w^{1} G^{o}$ & 8.16237 \\
\hline $3 s 3 p 3 d\left({ }^{4} D^{o}\right) 4 d$ & $u{ }^{5} F^{o}$ & 5.56736 & $3 s 3 p^{2}\left({ }^{2} P^{e}\right) 4 f$ & $v^{1} G^{o}$ & 7.30298 \\
\hline $3 s 3 p^{2}\left({ }^{4} P^{e}\right) 5 f$ & $t^{5} F^{o}$ & 4.79981 & $3 p^{3}\left({ }^{2} D^{o}\right) 4 d$ & $u^{1} G^{o}$ & 6.81251 \\
\hline $3 p 3 d^{2} 4 s$ & $s{ }^{5} F^{o}$ & 3.25576 & $3 s^{2} 3 p\left({ }^{2} P^{o}\right) 5 g$ & $t^{1} G^{o}$ & 6.76071 \\
\hline $3 s 3 d^{2} 4 p$ & $r^{5} F^{o}$ & 3.07932 & $3 s^{2} 3 d\left({ }^{2} D^{e}\right) 4 f$ & $s^{1} G^{o}$ & 6.60602 \\
\hline $3 s 3 p 3 d\left({ }^{4} F^{o}\right) 5 s$ & $q^{5} F^{o}$ & 2.77126 & $3 s 3 p 3 d\left(^{2} D^{o}\right) 4 d$ & $r^{1} G^{o}$ & 5.39592 \\
\hline $3 s 3 p^{2}\left({ }^{4} P^{e}\right) 6 f$ & $p^{5} F^{0}$ & 2.66604 & $3 s 3 p 3 d\left({ }^{2} F^{o}\right) 4 d$ & $q{ }^{1} G^{o}$ & 5.03421 \\
\hline $3 s 3 p 3 d\left({ }^{4} F^{o}\right) 5 d$ & ${ }^{5} F^{o}$ & 1.83091 & $3 s^{2} 3 p\left({ }^{2} P^{o}\right) 6 g$ & $p^{1} G^{o}$ & 4.72349 \\
\hline $3 d^{2} 4 s 4 p$ & $n^{5} F^{o}$ & 1.58764 & $3 s 3 p^{2}\left({ }^{2} D^{e}\right) 5 f$ & ${ }^{1} G^{\circ}$ & 4.19515 \\
\hline $3 s 3 p^{2}\left({ }^{4} P^{e}\right) 7 f$ & $m^{5} F^{o}$ & 1.38624 & $3 s^{2} 3 p\left({ }^{2} P^{o}\right) 7 g$ & $n{ }^{1} G^{o}$ & 3.49711 \\
\hline $3 s 3 p 3 d\left({ }^{4} P^{o}\right) 5 d$ & $l^{5} F^{o}$ & 1.25320 & $3 s 3 p^{2}\left({ }^{2} P^{e}\right) 5 f$ & $m^{1} G^{o}$ & 3.28148 \\
\hline $3 s 3 p 3 d\left({ }^{4} D^{\circ}\right) 5 d$ & $k^{5} F^{0}$ & 1.19476 & $3 p 3 d^{2} 4 s$ & $l^{1} G^{o}$ & 3.14076 \\
\hline $3 s 3 p 3 d\left({ }^{4} F^{o}\right) 5 g$ & $j^{5} F^{o}$ & 1.14171 & $3 s^{2} 3 d\left({ }^{2} D^{e}\right) 5 f$ & $k^{1} G^{o}$ & 2.67473 \\
\hline $3 s 3 p 3 d\left({ }^{4} P^{o}\right) 5 g$ & $i^{5} F^{\circ}$ & 0.71201 & $3 s 3 d^{2} 4 p$ & $j^{1} G^{o}$ & 2.66467 \\
\hline $3 s 3 p 3 d\left({ }^{4} D^{\circ}\right) 5 g$ & $h^{5} F^{o}$ & 0.63022 & $3 s^{2} 3 p\left({ }^{2} P^{o}\right) 8 g$ & $i{ }^{1} G^{o}$ & 2.62977 \\
\hline $3 s 3 p^{2}\left({ }^{4} P^{e}\right) 8 f$ & $g{ }^{5} F^{0}$ & 0.55688 & $3 p^{3}\left({ }^{2} D^{o}\right) 5 d$ & $h{ }^{1} G^{o}$ & 2.25697 \\
\hline $3 s 3 p^{2}\left({ }^{2} D^{e}\right) 3 d$ & $a^{1} G^{e}$ & 19.68440 & $3 s^{2} 3 p\left({ }^{2} P^{o}\right) 9 g$ & $g^{1} G^{o}$ & 2.09523 \\
\hline $3 s^{2} 3 d^{2}$ & $b{ }^{1} G^{e}$ & 17.52760 & $3 s 3 p^{2}\left({ }^{2} D^{e}\right) 6 f$ & $f^{1} G^{o}$ & 2.05146 \\
\hline $3 p^{2} 3 d^{2}$ & $c{ }^{1} G^{e}$ & 13.59030 & $3 s 3 p^{2}\left({ }^{2} D^{e}\right) 6 h$ & $e^{1} G^{o}$ & 1.97666 \\
\hline $3 p^{2} 3 d^{2}$ & $d^{1} G^{e}$ & 12.76820 & $3 p^{3}\left({ }^{2} D^{o}\right) 5 g$ & $d^{1} G^{o}$ & 1.83164 \\
\hline $3 p^{2} 3 d^{2}$ & $e^{1} G^{e}$ & 11.94650 & $3 s^{2} 3 p\left({ }^{2} P^{\circ}\right) 10 g$ & $c{ }^{1} G^{o}$ & 1.69807 \\
\hline $3 p^{2} 3 d^{2}$ & $f^{1} G^{e}$ & 11.26780 & $3 s 3 p^{2}\left(^{2} D^{e}\right) 3 d$ & $a^{3} G^{e}$ & 19.83320 \\
\hline $3 s^{2} 3 p\left({ }^{2} p^{o}\right) 4 f$ & $g^{1} G^{e}$ & 10.64852 & $3 p^{2} 3 d^{2}$ & $b^{3} G^{e}$ & 13.47890 \\
\hline $3 s 3 p^{2}\left({ }^{2} D^{e}\right) 4 d$ & $h^{1} G^{e}$ & 8.90584 & $3 p^{2} 3 d^{2}$ & $c^{3} G^{e}$ & 12.66430 \\
\hline $3 s 3 d^{2} 4 s$ & $i{ }^{1} G^{e}$ & 7.23462 & $3 p^{2} 3 d^{2}$ & $d^{3} G^{e}$ & 12.45730 \\
\hline $3 s^{2} 3 p\left({ }^{2} P^{o}\right) 5 f$ & $j{ }^{1} G^{e}$ & 6.83898 & $3 s^{2} 3 p\left({ }^{2} P^{o}\right) 4 f$ & $e^{3} G^{e}$ & 10.91020 \\
\hline $3 s 3 p 3 d\left({ }^{2} F^{o}\right) 4 p$ & $k^{1} G^{e}$ & 6.04576 & $3 s 3 p^{2}\left({ }^{2} D^{e}\right) 4 d$ & $f^{3} G^{e}$ & 8.99603 \\
\hline $3 p^{3}\left({ }^{2} D^{o}\right) 4 f$ & $l^{1} G^{e}$ & 5.72207 & $3 s^{2} 3 d\left({ }^{2} D^{e}\right) 4 d$ & $g^{3} G^{e}$ & 7.50435 \\
\hline $3 s^{2} 3 d\left({ }^{2} D^{e}\right) 4 d$ & $m^{1} G^{e}$ & 5.15630 & $3 s 3 p 3 d\left({ }^{4} F^{0}\right) 4 p$ & $h^{3} G^{e}$ & 7.12864 \\
\hline $3 s^{2} 3 p\left({ }^{2} P^{o}\right) 6 f$ & $n{ }^{1} G^{e}$ & 4.74972 & $3 s^{2} 3 p\left({ }^{2} P^{o}\right) 5 f$ & $i^{3} G^{e}$ & 6.93770 \\
\hline $3 s^{2} 3 p\left({ }^{2} P^{o}\right) 6 h$ & $o^{1} G^{e}$ & 4.69656 & $3 s 3 p 3 d\left({ }^{2} F^{0}\right) 4 p$ & $j^{3} G^{e}$ & 6.48123 \\
\hline $3 s 3 p^{2}\left({ }^{2} D^{e}\right) 5 d$ & $p^{1} G^{e}$ & 4.62351 & $3 p^{3^{2}}\left({ }^{2} D^{o}\right) 4 f$ & $k^{3} G^{e}$ & 5.99790 \\
\hline $3 p^{3}\left({ }^{2} P^{o}\right) 4 f$ & ${ }^{1} G^{e}$ & 4.39107 & $3 p^{3}\left({ }^{2} P^{o}\right) 4 f$ & $l^{3} G^{e}$ & 5.29692 \\
\hline $3 s 3 p 3 d\left({ }^{2} D^{\circ}\right) 4 f$ & $r^{1} G^{e}$ & 4.25690 & $3 s 3 p 3 d\left({ }^{4} F^{\circ}\right) 4 f$ & $m^{3} G^{e}$ & 5.21179 \\
\hline $3 s 3 p 3 d\left({ }^{2} F^{o}\right) 4 f$ & $s{ }^{1} G^{e}$ & 4.18962 & $3 s^{2} 3 p\left({ }^{2} P^{o}\right) 6 f$ & $n^{3} G^{e}$ & 4.80442 \\
\hline $3 s 3 p^{2}\left({ }^{2} D^{e}\right) 5 g$ & $t{ }^{1} G^{e}$ & 3.93569 & $3 s 3 p 3 d\left({ }^{4} P^{o}\right) 4 f$ & $o^{3} G^{e}$ & 4.74469 \\
\hline $3 s^{2} 3 p\left({ }^{2} P^{o}\right) 7 h$ & $u^{1} G^{e}$ & 3.48865 & $3 s^{2} 3 p\left({ }^{2} P^{o}\right) 6 h$ & $p{ }^{3} G^{e}$ & 4.69718 \\
\hline $3 s^{2} 3 p\left({ }^{2} P^{o}\right) 7 f$ & $v^{1} G^{e}$ & 3.48384 & $3 s 3 p 3 d\left({ }^{4} D^{o}\right) 4 f$ & $q^{3} G^{e}$ & 4.67920 \\
\hline $3 s 3 p^{2}\left({ }^{2} S^{e}\right) 5 g$ & $w^{1} G^{e}$ & 3.40954 & $3 s 3 p^{2}\left({ }^{2} D^{e}\right) 5 d$ & $r^{3} G^{e}$ & 4.59952 \\
\hline $3 s 3 p^{2}\left({ }^{2} P^{e}\right) 5 g$ & $x^{1} G^{e}$ & 3.12347 & $3 s 3 p 3 d\left({ }^{2} D^{o}\right) 4 f$ & $s^{3} G^{e}$ & 4.47174 \\
\hline $3 s^{2} 3 d\left({ }^{2} D^{e}\right) 5 d$ & $y^{1} G^{e}$ & 2.89080 & $3 s 3 d^{2} 4 s$ & $t^{3} G^{e}$ & 4.34432 \\
\hline $3 s^{2} 3 p\left({ }^{2} P^{o}\right) 8 f$ & $z^{1} G^{e}$ & 2.66599 & $3 s 3 p^{2}\left({ }^{4} P^{e}\right) 5 g$ & $u^{3} G^{e}$ & 4.27336 \\
\hline $3 s^{2} 3 p\left({ }^{2} P^{o}\right) 8 h$ & ${ }^{1} G^{e}$ & 2.63697 & $3 s 3 p 3 d\left({ }^{2} F^{o}\right) 4 f$ & $v^{3} G^{e}$ & 4.20779 \\
\hline $3 s^{2} 3 d\left({ }^{2} D^{e}\right) 5 g$ & ${ }^{1} G^{e}$ & 2.45294 & $3 s 3 p^{2}\left({ }^{2} D^{e}\right) 5 g$ & $w^{3} G^{e}$ & 3.98997 \\
\hline $3 s 3 p^{2}\left({ }^{2} D^{e}\right) 6 d$ & ${ }^{1} G^{e}$ & 2.25806 & $3 s^{2} 3 p\left({ }^{2} P^{o}\right) 7 f$ & $x^{3} G^{e}$ & 3.51604 \\
\hline $3 s^{2} 3 p\left({ }^{2} P^{o}\right) 9 f$ & ${ }^{1} G^{e}$ & 2.10519 & $3 s^{2} 3 p\left({ }^{2} P^{o}\right) 7 h$ & $y^{3} G^{e}$ & 3.49377 \\
\hline $3 s^{2} 3 p\left({ }^{2} P^{o}\right) 9 h$ & ${ }^{1} G^{e}$ & 2.08402 & $3 s 3 p^{2}\left({ }^{2} S^{e}\right) 5 g$ & $z^{3} G^{e}$ & 3.41471 \\
\hline $3 s 3 p^{2}\left({ }^{2} D^{e}\right) 6 g$ & ${ }^{1} G^{e}$ & 1.99869 & $3 s 3 p^{2}\left({ }^{2} P^{e}\right) 5 g$ & ${ }^{3} G^{e}$ & 3.13319 \\
\hline $3 p^{3}\left({ }^{2} D^{\circ}\right) 5 f$ & ${ }^{1} G^{e}$ & 1.88894 & $3 s^{2} 3 d\left({ }^{2} D^{e}\right) 5 d$ & ${ }^{3} G^{e}$ & 3.00098 \\
\hline $3 s^{2} 3 p\left({ }^{2} P^{o}\right) 10 f$ & ${ }^{1} G^{e}$ & 1.70366 & $3 s^{2} 3 p\left({ }^{2} P^{o}\right) 8 f$ & ${ }^{3} G^{e}$ & 2.68572 \\
\hline
\end{tabular}

TABLE I. Bound States of Fe XIII See page 137 for Explanation of Tables 


\begin{tabular}{|c|c|c|c|c|c|}
\hline \multicolumn{2}{|l|}{ Term } & $E(R y)$ & \multicolumn{2}{|l|}{ Term } & $E(R y)$ \\
\hline $3 s^{2} 3 p\left({ }^{2} P^{o}\right) 8 h$ & ${ }^{3} G^{e}$ & 2.63723 & $3 s 3 p^{2}\left({ }^{4} P^{e}\right) 7 g$ & $j^{5} G^{e}$ & 1.32628 \\
\hline $3 s 3 p^{2}\left({ }^{4} P^{e}\right) 6 g$ & ${ }^{3} G^{e}$ & 2.54401 & $3 s 3 p 3 d\left({ }^{4} P^{o}\right) 5 f$ & $k^{5} G^{e}$ & 0.89532 \\
\hline $3 s^{2} 3 d\left(^{2} D^{e}\right) 5 g$ & ${ }^{3} G^{e}$ & 2.46713 & $\left.3 s 3 p 3 d{ }^{4} D^{o}\right) 5 f$ & $l^{5} G^{e}$ & 0.82960 \\
\hline $3 s 3 p^{2}\left({ }^{2} D^{e}\right) 6 d$ & ${ }^{3} G^{e}$ & 2.30203 & $3 s 3 p^{2}\left({ }^{4} P^{e}\right) 8 g$ & $m^{5} G^{e}$ & 0.51444 \\
\hline $3 s 3 p 3 d\left({ }^{4} F^{\circ}\right) 5 p$ & ${ }^{3} G^{e}$ & 2.22865 & $3 s 3 p 3 d^{2}$ & $z{ }^{5} G^{o}$ & 16.89900 \\
\hline $3 s^{2} 3 p\left({ }^{2} P^{o}\right) 9 f$ & ${ }^{3} G^{e}$ & 2.11821 & $3 s 3 p^{2}\left({ }^{4} P^{e}\right) 4 f$ & $y{ }^{5} G^{o}$ & 8.87429 \\
\hline $3 s^{2} 3 p\left({ }^{2} P^{o}\right) 9 h$ & ${ }^{3} G^{e}$ & 2.08465 & $3 s 3 p 3 d\left({ }^{4} F^{\circ}\right) 4 d$ & ${ }^{9}{ }^{5} G^{0}$ & 6.11853 \\
\hline $3 p^{3}\left({ }^{2} D^{\circ}\right) 5 f$ & ${ }^{3} G^{e}$ & 2.03449 & $3 s 3 p 3 d\left({ }^{4} D^{o}\right) 4 d$ & $w^{5} G^{0}$ & 5.68388 \\
\hline $3 s 3 p^{2}\left({ }^{2} D^{e}\right) 6 g$ & ${ }^{3} G^{e}$ & 1.97165 & $3 s 3 p^{2}\left({ }^{4} P^{e}\right) 5 f$ & $v^{5} G^{o}$ & 4.85622 \\
\hline $3 s^{2} 3 p\left(^{2} P^{o}\right) 10 f$ & ${ }^{3} G^{e}$ & 1.71310 & $3 p 3 d^{2} 4 s$ & $u^{5} G^{o}$ & 3.25171 \\
\hline $3 s^{2} 3 p\left(^{2} P^{\circ}\right) 10 h$ & ${ }^{3} G^{e}$ & 1.69069 & $3 s 3 p^{2}\left({ }^{4} P^{e}\right) 6 f$ & $t^{5} G^{o}$ & 2.69517 \\
\hline $3 p^{3}\left({ }^{2} D^{o}\right) 3 d$ & $z^{3} G^{o}$ & 17.39280 & $3 s 3 p^{2}\left({ }^{4} P^{e}\right) 6 h$ & $s{ }^{5} G^{o}$ & 2.59150 \\
\hline $3 s 3 p 3 d^{2}$ & $y{ }^{3} G^{o}$ & 15.75620 & $3 s 3 d^{2} 4 p$ & $r^{5} G^{o}$ & 1.75293 \\
\hline $3 s 3 p 3 d^{2}$ & $x^{3} G^{0}$ & 15.54140 & $3 s 3 p 3 d\left({ }^{4} F^{\circ}\right) 5 d$ & $q^{5} G^{o}$ & 1.68045 \\
\hline $3 s 3 p 3 d^{2}$ & $w^{3} G^{o}$ & 14.73050 & $3 p^{3}\left({ }^{4} S^{o}\right) 5 g$ & $p^{5} G^{o}$ & 1.46836 \\
\hline $3 s 3 p^{2}\left({ }^{4} P^{e}\right) 4 f$ & $v^{3} G^{o}$ & 8.64909 & $3 s 3 p^{2}\left({ }^{4} P^{e}\right) 7 f$ & ${ }^{5} G^{o}$ & 1.40213 \\
\hline $3 s 3 p^{2}\left({ }^{2} D^{e}\right) 4 f$ & $u^{3} G^{0}$ & 8.19833 & $3 s 3 p^{2}\left({ }^{4} P^{e}\right) 7 h$ & $n^{5} G^{0}$ & 1.30334 \\
\hline $3 s 3 p^{2}\left({ }^{2} P^{e}\right) 4 f$ & $t^{3} G^{o}$ & 7.42746 & $3 s 3 p 3 d\left({ }^{4} D^{o}\right) 5 d$ & $m^{5} G^{o}$ & 1.21944 \\
\hline $3 p^{3^{3}}\left({ }^{2} D^{o}\right) 4 d$ & $s^{3} G^{o}$ & 6.81757 & $3 s 3 p 3 d\left({ }^{4} F^{\circ}\right) 5 g$ & $l^{5} G^{\circ}$ & 1.16983 \\
\hline $3 s^{2} 3 p\left({ }^{2} P^{\circ}\right) 5 q$ & $r^{3} G^{o}$ & 6.82942 & $3 s 3 p 3 d\left({ }^{4} P^{o}\right) 5 g$ & $k^{5} G^{o}$ & 0.72527 \\
\hline $3 s^{2} 3 d\left(^{2} D^{e}\right) 4 f$ & $q^{3} G^{o}$ & 6.39959 & $\left.3 s 3 p 3 d{ }^{4} D^{o}\right) 5 q$ & ${ }^{5} G^{o}$ & 0.65496 \\
\hline $3 s 3 p 3 d\left({ }^{4} F^{\circ}\right) 4 d$ & $p^{3} G^{o}$ & 6.17846 & $3 s 3 p^{2}\left({ }^{4} P^{e}\right) 8 f$ & $i^{5} G^{o}$ & 0.56775 \\
\hline $3 s 3 p 3 d\left({ }^{4} D^{o}\right) 4 d$ & $o^{3} G^{o}$ & 5.57773 & $3 s 3 p^{2}\left({ }^{4} P^{e}\right) 8 h$ & $h^{5} G^{0}$ & 0.51268 \\
\hline $3 s 3 p 3 d\left({ }^{2} D^{o}\right) 4 d$ & $n^{3} G^{o}$ & 5.37055 & $3 p^{2} 3 d^{2}$ & $a^{1} H^{e}$ & 12.84600 \\
\hline $3 s 3 p 3 d\left({ }^{2} F^{\circ}\right) 4 d$ & $m^{3} G^{o}$ & 5.07115 & $3 p^{3}\left({ }^{2} D^{o}\right) 4 f$ & $b{ }^{1} H^{e}$ & 5.94271 \\
\hline $3 s 3 p^{2}\left({ }^{4} P^{e}\right) 5 f$ & $l^{3} G^{\circ}$ & 4.75893 & $3 s^{2} 3 p\left({ }^{2} P^{o}\right) 6 h$ & $c{ }^{1} H^{e}$ & 4.72086 \\
\hline $3 s^{2} 3 p\left({ }^{2} P^{o}\right) 6 g$ & $k^{3} G^{o}$ & 4.71440 & $3 s 3 p 3 d\left({ }^{2} D^{o}\right) 4 f$ & $d^{1} H^{e}$ & 4.60691 \\
\hline $3 s 3 p^{2}\left({ }^{2} D^{e}\right) 5 f$ & $j^{3} G^{o}$ & 4.22335 & $3 s 3 p 3 d\left({ }^{2} F^{o}\right) 4 f$ & $e^{1} H^{e}$ & 4.06806 \\
\hline $3 s^{2} 3 p\left({ }^{2} P^{\circ}\right) 7 g$ & $i^{3} G^{o}$ & 3.49889 & $3 s 3 p^{2}\left({ }^{2} D^{e}\right) 5 g$ & $f^{1} H^{e}$ & 3.98884 \\
\hline $3 s 3 p^{2}\left({ }^{2} P^{e}\right) 5 f$ & $h^{3} G^{0}$ & 3.34232 & $3 s^{2} 3 p\left({ }^{2} P^{o}\right) 7 h$ & ${ }^{1} H^{e}$ & 3.48060 \\
\hline $3 p 3 d^{2} 4 s$ & $g^{3} G^{o}$ & 3.30557 & $3 s 3 p^{2}\left({ }^{2} P^{e}\right) 5 g$ & ${ }^{g}{ }^{1} H^{e}$ & 3.17621 \\
\hline $3 p 3 d^{2} 4 s$ & $f^{3} G^{0}$ & 2.96634 & $3 s^{2} 3 p\left({ }^{2} P^{\circ}\right) 8 h$ & $i^{1} H^{e}$ & 2.64822 \\
\hline $3 s^{2} 3 p\left({ }^{2} P^{o}\right) 8 g$ & $e^{3} G^{o}$ & 2.66422 & $3 s^{2} 3 d\left({ }^{2} D^{e}\right) 5 g$ & $j{ }^{1} H^{e}$ & 2.47389 \\
\hline $3 s 3 p^{2}\left({ }^{4} P^{e}\right) 6 f$ & $d^{3} G^{o}$ & 2.64610 & $3 s^{2} 3 p\left({ }^{2} P^{o}\right) 9 h$ & $k^{1} H^{e}$ & 2.09098 \\
\hline $3 s 3 p^{2}\left({ }^{4} P^{e}\right) 6 h$ & $c^{3} G^{0}$ & 2.59132 & $3 p^{3}\left(^{2} D^{\circ}\right) 5 f$ & $l^{1} H^{e}$ & 2.04219 \\
\hline $3 s^{2} 3 d\left(^{2} D^{e}\right) 5 f$ & $b^{3} G^{o}$ & 2.55790 & $3 s 3 p^{2}\left({ }^{2} D^{e}\right) 6 g$ & $m^{1} H^{e}$ & 1.94256 \\
\hline $3 p^{3}\left(^{2} D^{\circ}\right) 5 d$ & $a^{3} G^{o}$ & 2.53666 & $3 s^{2} 3 p\left(^{2} P^{o}\right) 10 h$ & $n^{1} H^{e}$ & 1.69401 \\
\hline $3 p 3 d^{2} 4 s$ & ${ }^{3} G^{o}$ & 2.26268 & $3 s 3 p 3 d^{2}$ & $z^{1} H^{o}$ & 14.82730 \\
\hline $3 s^{2} 3 p\left({ }^{2} P^{o}\right) 9 g$ & ${ }^{3} G^{o}$ & 2.09518 & $3 s 3 p^{2}\left({ }^{2} D^{e}\right) 4 f$ & $y{ }^{1} H^{o}$ & 7.98613 \\
\hline $3 s 3 p^{2}\left(^{2} D^{e}\right) 6 f$ & ${ }^{3} G^{o}$ & 2.07027 & $3 s^{2} 3 p\left({ }^{2} P^{o}\right) 5 q$ & $x^{1} H^{o}$ & 6.85377 \\
\hline $3 s 3 p^{2}\left(^{2} D^{e} 6 h\right.$ & ${ }^{3} G^{o}$ & 1.97746 & $3 s^{2} 3 d\left({ }^{2} D^{e}\right) 4 f$ & $w^{1} H^{\circ}$ & 6.12924 \\
\hline $3 p^{3}\left({ }^{2} D^{o}\right) 5 g$ & ${ }^{3} G^{o}$ & 1.84765 & $3 s 3 p 3 d\left({ }^{2} F^{0}\right) 4 d$ & $v{ }^{1} H^{o}$ & 4.96909 \\
\hline $3 s 3 d^{2} 4 p$ & ${ }^{3} G^{o}$ & 1.83444 & $3 s^{2} 3 p\left({ }^{2} P^{o}\right) 6 g$ & $u^{1} H^{o}$ & 4.67814 \\
\hline $3 s 3 p 3 d\left({ }^{4} F^{\circ}\right) 5 d$ & ${ }^{3} G^{o}$ & $\begin{array}{l}1.078008 \\
1.78\end{array}$ & $3 s 3 p^{2}\left({ }^{2} D^{e}\right) 5 f$ & $t^{1} H^{o}$ & 4.12563 \\
\hline $3 s^{2} 3 p\left({ }^{2} P^{o}\right) 10 g$ & ${ }^{3} G^{o}$ & 1.69699 & $3 s^{2} 3 p\left({ }^{2} P^{o}\right) 7 g$ & $s{ }^{1} H^{o}$ & 3.45268 \\
\hline $3 p^{2} 3 d^{2}$ & $a{ }^{5} G^{e}$ & 14.13040 & $3 s^{2} 3 p\left({ }^{2} P^{o}\right) 7 i$ & $r^{1} H^{o}$ & 3.44831 \\
\hline $3 s 3 p 3 d\left({ }^{4} F^{\circ}\right) 4 p$ & $b^{5} G^{e}$ & 7.55521 & $3 s^{2} 3 p\left({ }^{2} P^{o}\right) 8 g$ & $q^{1} H^{\circ}$ & 2.66510 \\
\hline $3 s 3 p 3 d\left({ }^{4} F^{o}\right) 4 f$ & $c^{5} G^{e}$ & 5.33883 & $3 s^{2} 3 p\left({ }^{2} P^{0}\right) 8 i$ & $p^{1} H^{o}$ & 2.64105 \\
\hline $3 s 3 p 3 d\left({ }^{4} P^{o}\right) 4 f$ & $d^{5} G^{e}$ & 4.97816 & $3 s^{2} 3 d\left(^{2} D^{e}\right) 5 f$ & ${ }^{1} H^{o}$ & 2.50417 \\
\hline $\left.3 s 3 p 3 d{ }^{4} D^{\circ}\right) 4 f$ & $e^{5} G^{e}$ & 4.79917 & $3 p 3 d^{2} 4 s$ & $n^{1} H^{\circ}$ & 2.44288 \\
\hline $3 s 3 p^{2}\left({ }^{4} P^{e}\right) 5 g$ & $f^{5} G^{e}$ & 4.53770 & $3 s^{2} 3 p\left({ }^{2} P^{o}\right) 9 i$ & $m^{1} H^{o}$ & 2.08751 \\
\hline $3 s 3 p^{2}\left({ }^{4} P^{e}\right) 6 g$ & $g^{5} G^{e}$ & 2.56580 & $3 s^{2} 3 p\left({ }^{2} P^{o}\right) 9 g$ & $l^{1} H^{o}$ & 2.07891 \\
\hline $3 s 3 p 3 d\left({ }^{4} F^{0}\right) 5 p$ & $h^{5} G^{e}$ & 2.36628 & $3 s 3 p^{2}\left({ }^{2} D^{e}\right) 6 f$ & $k^{1} H^{o}$ & 2.01933 \\
\hline $3 s 3 p 3 d\left({ }^{4} F^{0}\right) 5 f$ & $i^{5} G^{e}$ & 1.33794 & $3 s 3 p^{2}\left({ }^{2} D^{e}\right) 6 h$ & $j{ }^{1} H^{o}$ & 1.99377 \\
\hline
\end{tabular}

TABLE I. Bound States of Fe XIII

See page 137 for Explanation of Tables 


\begin{tabular}{|c|c|c|c|c|c|}
\hline \multicolumn{2}{|l|}{ Term } & \multirow{2}{*}{$\begin{array}{r}E(R y) \\
1.83167\end{array}$} & \multicolumn{2}{|l|}{ Term } & \multirow{2}{*}{$\begin{array}{r}E(R y) \\
2.56361\end{array}$} \\
\hline $3 p^{3}\left({ }^{2} D^{o}\right) 5 g$ & $i^{1} H^{o}$ & & $3 s 3 p^{2}\left({ }^{4} P^{e}\right) 6 h$ & $y^{5} H^{o}$ & \\
\hline $3 s^{2} 3 p\left({ }^{2} P^{o}\right) 10 i$ & $h{ }^{1} H^{o}$ & 1.69126 & $3 s 3 p 3 d\left({ }^{4} F^{o}\right) 5 d$ & $x^{5} H^{o}$ & 1.71740 \\
\hline $3 p^{2} 3 d^{2}$ & $a^{3} H^{e}$ & 13.62250 & $3 s 3 p^{2}\left({ }^{4} P^{e}\right) 7 h$ & $w^{5} H^{\circ}$ & 1.32699 \\
\hline $3 p^{2} 3 d^{2}$ & $b^{3} H^{e}$ & 12.89100 & $3 s 3 p 3 d\left({ }^{4} F^{o}\right) 5 g$ & $v^{5} H^{o}$ & 1.18451 \\
\hline $3 p^{3}\left({ }^{2} D^{o}\right) 4 f$ & $c^{3} H^{e}$ & 5.97902 & $3 s 3 p 3 d\left({ }^{4} P^{o}\right) 5 g$ & $u^{5} H^{o}$ & 0.71322 \\
\hline $3 s 3 p 3 d\left({ }^{4} F^{o}\right) 4 f$ & $d^{3} H^{e}$ & 5.23226 & $3 s 3 p 3 d\left({ }^{4} D^{o}\right) 5 g$ & $t^{5} H^{\circ}$ & 0.66117 \\
\hline $3 s 3 p 3 d\left({ }^{4} D^{o}\right) 4 f$ & $e^{3} H^{e}$ & 4.86606 & $3 s 3 p^{2}\left({ }^{4} P^{e}\right) 8 h$ & $s{ }^{5} H^{o}$ & 0.49520 \\
\hline $3 s^{2} 3 p\left({ }^{2} P^{o}\right) 6 h$ & $f^{3} H^{e}$ & 4.72084 & $3 p^{2} 3 d^{2}$ & $a{ }^{1} I^{e}$ & 12.93450 \\
\hline $3 s 3 p 3 d\left({ }^{2} D^{o}\right) 4 f$ & $g^{3} H^{e}$ & 4.60816 & $3 s^{2} 3 p\left({ }^{2} P^{o}\right) 6 h$ & $b^{1} I^{e}$ & 4.70152 \\
\hline $3 s 3 p^{2}\left({ }^{4} P^{e}\right) 5 g$ & $h^{3} H^{e}$ & 4.39426 & $3 s 3 p^{2}\left({ }^{2} D^{e}\right) 5 g$ & $c^{1} I^{e}$ & 4.11118 \\
\hline $3 s 3 p 3 d\left({ }^{2} F^{o}\right) 4 f$ & $i^{3} H^{e}$ & 4.21676 & $3 s 3 p^{2}\left({ }^{2} D^{e}\right) 6 g$ & $d^{1} I^{e}$ & 3.90583 \\
\hline $3 s 3 p^{2}\left({ }^{2} D^{e}\right) 5 g$ & $j^{3} H^{e}$ & 3.97312 & $3 s^{2} 3 p\left({ }^{2} P^{o}\right) 7 h$ & $e^{1} I^{e}$ & 3.45007 \\
\hline $3 s^{2} 3 p\left({ }^{2} P^{o}\right) 7 h$ & $k^{3} H^{e}$ & 3.48022 & $3 s^{2} 3 p\left(^{2} P^{o}\right) 8 h$ & $f^{1} I^{e}$ & 2.65351 \\
\hline $3 s 3 p^{2}\left({ }^{2} P^{e}\right) 5 g$ & $l^{3} H^{e}$ & 3.17908 & $3 s^{2} 3 p\left({ }^{2} P^{o}\right) 8 k$ & $g^{1} I^{e}$ & 2.64035 \\
\hline $3 s^{2} 3 p\left({ }^{2} P^{o}\right) 8 h$ & $m^{3} H^{e}$ & 2.64844 & $3 s^{2} 3 d\left({ }^{2} D^{e}\right) 5 g$ & $h^{1} I^{e}$ & 2.39908 \\
\hline $3 s 3 p^{2}\left({ }^{4} P^{e}\right) 6 g$ & $n^{3} H^{e}$ & 2.57711 & $3 s^{2} 3 p\left(^{2} P^{o}\right) 9 k$ & $i^{1} I^{e}$ & 2.08623 \\
\hline $\left.3 s^{2} 3 d d^{2} D^{e}\right) 5 g$ & $o^{3} H^{e}$ & 2.46675 & $3 s^{2} 3 p\left(^{2} P^{o}\right) 9 h$ & $j^{1} I^{e}$ & 2.08176 \\
\hline $3 s^{2} 3 p\left({ }^{2} P^{o}\right) 9 h$ & $p^{3} H^{e}$ & 2.09068 & $3 s 3 p^{2}\left({ }^{2} D^{e}\right) 7 g$ & $k^{1} I^{e}$ & 1.95185 \\
\hline $3 p^{3}\left({ }^{2} D^{o}\right) 5 f$ & $q^{3} H^{e}$ & 2.02947 & $3 s^{2} 3 p\left({ }^{2} P^{o}\right) 7 i$ & $z^{1} I^{o}$ & 3.45551 \\
\hline $3 s 3 p^{2}\left({ }^{2} D^{e}\right) 6 g$ & $r^{3} H^{e}$ & 1.97611 & $3 s^{2} 3 p\left({ }^{2} P^{o}\right) 8 i$ & $y{ }^{1} I^{o}$ & 2.64583 \\
\hline $3 s^{2} 3 p\left({ }^{2} P^{o}\right) 10 h$ & $s^{3} H^{e}$ & 1.69381 & $3 s^{2} 3 p\left({ }^{2} P^{o}\right) 9 i$ & $x^{1} I^{\circ}$ & 2.09175 \\
\hline $3 s 3 p 3 d^{2}$ & $z^{3} H^{\circ}$ & 15.91400 & $3 s 3 p^{2}\left({ }^{2} D^{e}\right) 6 h$ & $w^{1} I^{o}$ & 1.98721 \\
\hline $3 s 3 p^{2}\left({ }^{2} D^{e}\right) 4 f$ & $y^{3} H^{\circ}$ & 8.13356 & $3 p^{3}\left({ }^{2} D^{\circ}\right) 5 g$ & $v^{1} I^{o}$ & 1.85550 \\
\hline $3 s^{2} 3 d\left(^{2} D^{e}\right) 4 f$ & $x^{3} H^{o}$ & 6.92029 & $3 s^{2} 3 p\left(^{2} P^{o}\right) 10 i$ & $u^{1} I^{\circ}$ & 1.69273 \\
\hline $3 s^{2} 3 p\left({ }^{2} P^{o}\right) 5 g$ & $w^{3} H^{o}$ & 6.52399 & $3 s 3 p 3 d\left({ }^{4} F^{o}\right) 4 f$ & $a^{3} I^{e}$ & 4.92032 \\
\hline $3 s 3 p 3 d\left({ }^{4} F^{o}\right) 4 d$ & $v^{3} H^{\circ}$ & 5.89446 & $3 s^{2} 3 p\left(^{2} P^{o}\right) 6 h$ & $b^{3} I^{e}$ & 4.70214 \\
\hline $\left.3 s 3 p 3 d d^{2} F^{o}\right) 4 d$ & $u^{3} H^{o}$ & 5.04326 & $3 s 3 p 3 d\left({ }^{2} F^{o}\right) 4 f$ & $c^{3} I^{e}$ & 4.31338 \\
\hline $3 s^{2} 3 p\left({ }^{2} P^{o}\right) 6 g$ & $t^{3} H^{o}$ & 4.69858 & $3 s 3 p^{2}\left({ }^{2} D^{e}\right) 5 g$ & $d^{3} I^{e}$ & 3.94389 \\
\hline $3 s 3 p^{2}\left({ }^{2} D^{e}\right) 5 f$ & $s{ }^{3} H^{o}$ & 4.18792 & $3 s^{2} 3 p\left({ }^{2} P^{o}\right) 7 h$ & $e^{3} I^{e}$ & 3.45130 \\
\hline $3 s^{2} 3 p\left({ }^{2} P^{o}\right) 7 g$ & $r^{3} H^{o}$ & 3.45858 & $3 s^{2} 3 p\left(^{2} P^{o}\right) 8 h$ & $f^{3} I^{e}$ & 2.65546 \\
\hline $3 s^{2} 3 p\left({ }^{2} P^{o}\right) 7 i$ & $q^{3} H^{o}$ & 3.44836 & $3 s^{2} 3 p\left({ }^{2} P^{o}\right) 8 k$ & $g^{3} I^{e}$ & 2.64035 \\
\hline $3 p 3 d^{2} 4 s$ & $p^{3} H^{o}$ & 2.74712 & $3 s^{2} 3 d\left({ }^{2} D^{e}\right) 5 g$ & $h^{3} I^{e}$ & 2.42910 \\
\hline $3 s^{2} 3 p\left({ }^{2} P^{o}\right) 8 g$ & ${ }^{3} H^{\circ}$ & 2.69002 & $3 s^{2} 3 p\left(^{2} P^{o}\right) 9 k$ & $i^{3} I^{e}$ & 2.08623 \\
\hline $3 s^{2} 3 p\left({ }^{2} P^{o}\right) 8 i$ & $n^{3} H^{o}$ & 2.64111 & $3 s^{2} 3 p\left({ }^{2} P^{o}\right) 9 h$ & $j^{3} I^{e}$ & 2.08392 \\
\hline $3 s^{2} 3 d\left(^{2} D^{e}\right) 5 f$ & $m^{3} H^{o}$ & 2.60333 & $3 s 3 p^{2}\left({ }^{2} D^{e}\right) 6 g$ & $k^{3} I^{e}$ & 1.95844 \\
\hline $3 s 3 p^{2}\left({ }^{4} P^{e}\right) 6 h$ & $l^{3} H^{o}$ & 2.56302 & $3 s^{2} 3 p\left({ }^{2} P^{o}\right) 7 i$ & $z^{3} I^{o}$ & 3.45551 \\
\hline $3 s^{2} 3 p\left({ }^{2} P^{o}\right) 9 g$ & $k^{3} H^{o}$ & 2.09457 & $3 s^{2} 3 p\left(^{2} P^{o}\right) 8 i$ & $y^{3} I^{o}$ & 2.64582 \\
\hline $3 s^{2} 3 p\left({ }^{2} P^{o}\right) 9 i$ & $j^{3} H^{o}$ & 2.08753 & $3 s 3 p^{2}\left({ }^{4} P^{e}\right) 6 h$ & $x^{3} I^{\circ}$ & 2.58408 \\
\hline $3 s 3 p^{2}\left({ }^{2} D^{e}\right) 6 f$ & $i^{3} H^{o}$ & 2.05065 & $3 s^{2} 3 p\left({ }^{2} P^{o}\right) 9 i$ & $w^{3} I^{o}$ & 2.09175 \\
\hline $3 s 3 p^{2}\left({ }^{2} D^{e}\right) 6 h$ & $h^{3} H^{o}$ & 1.99502 & $3 s 3 p^{2}\left({ }^{2} D^{e}\right) 6 h$ & $v^{3} I^{o}$ & 1.98625 \\
\hline $3 p^{3}\left({ }^{2} D^{o}\right) 5 g$ & $g^{3} H^{\circ}$ & 1.84701 & $3 p^{3}\left({ }^{2} D^{o}\right) 5 g$ & $u^{3} I^{\circ}$ & 1.85808 \\
\hline $3 s^{2} 3 p\left({ }^{2} P^{o}\right) 10 g$ & $f^{3} H^{o}$ & 1.69297 & $3 s^{2} 3 p\left({ }^{2} P^{o}\right) 10 i$ & $t^{3} I^{o}$ & 1.69272 \\
\hline $3 s^{2} 3 p\left(^{2} P^{o}\right) 10 i$ & $e^{3} H^{o}$ & 1.69123 & $3 s 3 p 3 d\left({ }^{4} F^{o}\right) 4 f$ & $a^{5} I^{e}$ & 5.44905 \\
\hline $3 s 3 p 3 d\left({ }^{4} F^{o}\right) 4 f$ & $a^{5} H^{e}$ & 5.30278 & $3 s 3 p 3 d\left({ }^{4} F^{o}\right) 5 f$ & $b^{5} I^{e}$ & 1.37288 \\
\hline $3 s 3 p 3 d\left({ }^{4} D^{\circ}\right) 4 f$ & $b^{5} H^{e}$ & 5.01252 & $3 s 3 p^{2}\left({ }^{4} P^{e}\right) 7 i$ & $c^{5} I^{e}$ & 1.32111 \\
\hline $3 s 3 p^{2}\left({ }^{4} P^{e}\right) 5 g$ & $c^{5} H^{e}$ & 4.57582 & $3 s 3 p^{2}\left({ }^{4} P^{e}\right) 8 i$ & $d^{5} I^{e}$ & 0.51433 \\
\hline $3 s 3 p^{2}\left({ }^{4} P^{e}\right) 6 g$ & $d^{5} H^{e}$ & 2.59460 & $3 s 3 p^{2}\left({ }^{4} P^{e}\right) 6 h$ & $z^{5} I^{o}$ & 2.58448 \\
\hline $3 s 3 p^{2}\left({ }^{4} P^{e}\right) 7 g$ & $e^{5} H^{e}$ & 1.34865 & $3 s 3 p^{2}\left({ }^{4} P^{e}\right) 7 h$ & $y^{5} I^{o}$ & 1.33917 \\
\hline $3 s 3 p^{2}\left({ }^{4} P^{e}\right) 7 i$ & $f^{5} H^{e}$ & 1.32923 & $3 s 3 p 3 d\left({ }^{4} F^{o}\right) 5 g$ & $x^{5} I^{0}$ & 1.17423 \\
\hline $3 s 3 p 3 d\left({ }^{4} F^{o}\right) 5 f$ & $g^{5} H^{e}$ & 1.31495 & $3 s 3 p 3 d\left({ }^{4} D^{\circ}\right) 5 g$ & $w^{5} I^{0}$ & 0.68953 \\
\hline $3 s 3 p 3 d\left({ }^{4} D^{\circ}\right) 5 f$ & $h^{5} H^{e}$ & 0.87954 & $3 s 3 p^{2}\left({ }^{4} P^{e}\right) 8 k$ & $v^{5} I^{o}$ & 0.51704 \\
\hline $3 s 3 p^{2}\left({ }^{4} P^{e}\right) 8 g$ & $i^{5} H^{e}$ & 0.52408 & $3 s 3 p^{2}\left({ }^{4} P^{e}\right) 8 h$ & $u^{5} I^{0}$ & 0.50162 \\
\hline $3 s 3 p^{2}\left({ }^{4} P^{e}\right) 8 i$ & $j^{5} H^{e}$ & 0.51985 & $3 s^{2} 3 p\left(^{2} P^{o}\right) 8 k$ & $a{ }^{1} K^{e}$ & 2.64200 \\
\hline $3 s 3 p 3 d\left({ }^{4} F^{o}\right) 4 d$ & $z^{5} H^{\circ}$ & 6.17884 & $3 s^{2} 3 p\left({ }^{2} P^{o}\right) 9 k$ & $b^{1} K^{e}$ & 2.08789 \\
\hline
\end{tabular}

TABLE I. Bound States of Fe XIII

See page 137 for Explanation of Tables 


\begin{tabular}{|c|c|c|c|c|c|}
\hline \multicolumn{2}{|l|}{ Term } & $E(R y)$ & \multicolumn{2}{|l|}{ Term } & $E(R y)$ \\
\hline $3 s^{2} 3 p\left({ }^{2} P^{o}\right) 10 k$ & $c{ }^{1} K^{e}$ & 1.69126 & $3 s 3 p 3 d\left({ }^{4} F^{o}\right) 5 g$ & $z^{5} K^{o}$ & 1.13140 \\
\hline $3 s^{2} 3 p\left({ }^{2} P^{o}\right) 7 i$ & $z^{1} K^{o}$ & 3.44933 & $3 s 3 p^{2}\left({ }^{4} P^{e}\right) 8 k$ & $y^{5} K^{o}$ & 0.51518 \\
\hline $3 s^{2} 3 p\left({ }^{2} P^{o}\right) 8 i$ & $y^{1} K^{o}$ & 2.64151 & $3 s^{2} 3 p\left({ }^{2} P^{o}\right) 8 k$ & $a^{1} L^{e}$ & 2.64055 \\
\hline $3 s^{2} 3 p\left({ }^{2} P^{o}\right) 9 i$ & $x^{1} K^{o}$ & 2.08831 & $3 s^{2} 3 p\left({ }^{2} P^{o}\right) 9 k$ & $b^{1} L^{e}$ & 2.08642 \\
\hline $3 s^{2} 3 p\left({ }^{2} P^{o}\right) 9 l$ & $w^{1} K^{o}$ & 2.08630 & $3 s^{2} 3 p\left({ }^{2} P^{o}\right) 10 k$ & $c{ }^{1} L^{e}$ & 1.68999 \\
\hline $3 s 3 p^{2}\left({ }^{2} D^{e}\right) 6 h$ & $v^{1} K^{o}$ & 1.94969 & $\left.3 s^{2} 3 p{ }^{2} P^{o}\right) 9 l$ & $z^{1} L^{\circ}$ & 2.08661 \\
\hline $3 s^{2} 3 p\left({ }^{2} P^{o}\right) 10 i$ & $u{ }^{1} K^{o}$ & 1.69035 & $3 s^{2} 3 p\left({ }^{2} P^{o}\right) 10 l$ & $y^{1} L^{o}$ & 1.69028 \\
\hline $3 s^{2} 3 p\left({ }^{2} P^{o}\right) 8 k$ & $a^{3} K^{e}$ & 2.64200 & $3 s^{2} 3 p\left({ }^{2} P^{o}\right) 8 k$ & $a^{3} L^{e}$ & 2.64055 \\
\hline $3 s^{2} 3 p\left({ }^{2} P^{o}\right) 9 k$ & $b^{3} K^{e}$ & 2.08789 & $3 s^{2} 3 p\left(^{2} P^{o}\right) 9 k$ & $b^{3} L^{e}$ & 2.08642 \\
\hline $3 s^{2} 3 p\left({ }^{2} P^{o}\right) 10 k$ & $c^{3} K^{e}$ & 1.69126 & $3 s^{2} 3 p\left({ }^{2} P^{o}\right) 10 k$ & $c^{3} L^{e}$ & 1.68999 \\
\hline $3 s^{2} 3 p\left({ }^{2} P^{o}\right) 7 i$ & $z^{3} K^{\circ}$ & 3.44934 & $3 s^{2} 3 p\left({ }^{2} P^{o}\right) 9 l$ & $z^{3} L^{o}$ & 2.08661 \\
\hline $3 s^{2} 3 p\left({ }^{2} P^{o}\right) 8 i$ & $y^{3} K^{o}$ & 2.64153 & $3 s^{2} 3 p\left({ }^{2} P^{\circ}\right) 10 l$ & $y^{3} L^{0}$ & 1.69028 \\
\hline $3 s^{2} 3 p\left({ }^{2} P^{o}\right) 9 i$ & $x^{3} K^{o}$ & 2.08835 & $3 s 3 p^{2}\left({ }^{4} P^{e}\right) 8 k$ & $z^{5} L^{0}$ & 0.51679 \\
\hline $3 s^{2} 3 p\left({ }^{2} P^{o}\right) 9 l$ & $w^{3} K^{o}$ & 2.08630 & $3 s^{2} 3 p\left({ }^{2} P^{o}\right) 10 m$ & $a{ }^{1} M^{e}$ & 1.69002 \\
\hline $3 s 3 p^{2}\left({ }^{2} D^{e}\right) 6 h$ & $v^{3} K^{o}$ & 1.95031 & $3 s^{2} 3 p\left(^{2} P^{o}\right) 9 l$ & $z^{1} M^{o}$ & 2.08639 \\
\hline $3 s^{2} 3 p\left({ }^{2} P^{o}\right) 10 i$ & $u^{3} K^{o}$ & 1.69035 & $3 s^{2} 3 p\left({ }^{2} P^{o}\right) 10 m$ & $a^{3} M^{e}$ & 1.69002 \\
\hline $3 s 3 p^{2}\left({ }^{4} P^{e}\right) 7 i$ & $a^{5} K^{e}$ & 1.32790 & $3 s^{2} 3 p\left({ }^{2} P^{o}\right) 9 l$ & $z^{3} M^{o}$ & 2.08639 \\
\hline $3 s 3 p^{2}\left({ }^{4} P^{e}\right) 8 i$ & $b^{5} K^{e}$ & 0.51880 & & & \\
\hline
\end{tabular}

TABLE II. Oscillator Strengths, Line Strengths, and Transition Probabilities for Allowed Transitions in Fe XIII See page 137 for Explanation of Tables 


\begin{tabular}{|c|c|c|c|c|c|c|c|c|}
\hline Transition & $\begin{array}{c}E_{\mathrm{i}} \\
\mathrm{Ry} / \mathrm{cm}^{1}\end{array}$ & $\begin{array}{c}\mathrm{E}_{\mathrm{j}} \\
\mathrm{Ry} / \mathrm{cm}^{1}\end{array}$ & $\begin{array}{l}\mathrm{E}_{\mathrm{il}} \\
\text { Ry }\end{array}$ & $g_{i}$ & $g_{j}$ & $f_{i j}$ & S & $\begin{array}{l}A_{i j} \\
s^{\prime}\end{array}$ \\
\hline$a^{1} S^{e} z^{1} P^{0}$ & $50 \Omega 000$ & 22.5443 & $3.158 \mathrm{E}+00$ & 1 & 3 & $1.858 \mathrm{E}-01$ & $1.765 \mathrm{E}-01$ & 4.962E+09 \\
\hline$a^{1} S^{e} y^{1} p^{\circ}$ & $\begin{array}{l}508.000 \\
25.7022\end{array}$ & $\begin{array}{r}438050.00 \\
21.3356\end{array}$ & $3.158 \mathrm{E}+00$ & 1 & 3 & $1.858 \mathrm{E}-01$ & $1.765 \mathrm{E}-01$ & $4.962 E+09$ \\
\hline & 91508.000 & $\begin{array}{r}21.3356 \\
570690.00\end{array}$ & $\begin{array}{l}4.367 E+00 \\
4.367 E+00\end{array}$ & $\begin{array}{l}1 \\
1\end{array}$ & $\begin{array}{l}3 \\
3\end{array}$ & $\begin{array}{l}1.097 E+00 \\
1.097 E+00\end{array}$ & $\begin{array}{l}7.539 \mathrm{E}-01 \\
7.539 \mathrm{E}-01\end{array}$ & $\begin{array}{l}5.602 E+10 \\
5.602 E+10\end{array}$ \\
\hline$a^{1} S^{\theta} \quad t^{1} p^{0}$ & 25.7022 & 14.1262 & $1.158 E+01$ & 1 & 3 & $8.512 \mathrm{E}-02$ & 2.206E-02 & 3. $054 \mathrm{E}+10$ \\
\hline$a^{1} S^{\theta} r^{1} p^{\circ}$ & $\begin{array}{r}1508.000 \\
25.7022\end{array}$ & $\begin{array}{r}1361830.00 \\
11.4945\end{array}$ & $\begin{array}{l}1.158 \mathrm{E}+01 \\
1.421 \mathrm{E}+01\end{array}$ & $\begin{array}{l}1 \\
1\end{array}$ & $\begin{array}{l}3 \\
3\end{array}$ & $\begin{array}{l}8.512 \mathrm{E}-02 \\
5.337 \mathrm{E}-01\end{array}$ & $\begin{array}{l}2.206 \mathrm{E}-02 \\
1.127 \mathrm{E}-01\end{array}$ & $\begin{array}{l}3.054 \mathrm{E}+10 \\
2.884 \mathrm{E}+11\end{array}$ \\
\hline & 91508.000 & 1650620.00 & $1.421 \mathrm{E}+01$ & 1 & 3 & 5.337E-01 & $1.127 \mathrm{E}-01$ & $2.884 \mathrm{E}+11$ \\
\hline$d^{1} p^{\theta} \quad r^{1} p^{0}$ & $\begin{array}{r}12.7280 \\
515260000\end{array}$ & $\begin{array}{r}11.4945 \\
165062000\end{array}$ & $\begin{array}{l}1.233 \mathrm{E}+00 \\
1.233 \mathrm{E}+00\end{array}$ & $\begin{array}{l}3 \\
3\end{array}$ & 3 & $2.019 \mathrm{E}-06$ & $\begin{array}{l}1.473 \mathrm{E}-05 \\
1.473 \mathrm{E}-05\end{array}$ & $\begin{array}{l}2.467 E+04 \\
2.467 E+04\end{array}$ \\
\hline$a^{1} D^{\theta} z^{1} P^{\circ}$ & 26.0981 & 22.5443 & $3.554 \mathrm{E}+00$ & 5 & 3 & $1.834 \mathrm{E}-01$ & $7.743 \mathrm{E}-01$ & $3.101 \mathrm{E}+10$ \\
\hline & 48068.000 & 438050.00 & $3.554 \mathrm{E}+00$ & 5 & 3 & $1.834 \mathrm{E}-01$ & $7.743 \mathrm{E}-01$ & 3. $101 \mathrm{E}+10$ \\
\hline$a^{1} D^{\theta}$ y ${ }^{1} p^{\circ}$ & $\begin{array}{r}26.0981 \\
48068.000\end{array}$ & $\begin{array}{r}21.3356 \\
570690.00\end{array}$ & $\begin{array}{l}4.762 E+00 \\
4.762 E+00\end{array}$ & $\begin{array}{l}5 \\
5\end{array}$ & 3 & $\begin{array}{l}5.767 \mathrm{E}-05 \\
5.767 \mathrm{E}-05\end{array}$ & $1.816 \mathrm{E}-04$ & $1.751 \mathrm{E}+07$ \\
\hline$a^{1} D^{e} \quad t^{1} P^{\circ}$ & 26.0981 & $\begin{array}{l}10690.00 \\
14.1262\end{array}$ & $1.197 \mathrm{E}+01$ & $\begin{array}{l}0 \\
5\end{array}$ & 3 & $1.184 \mathrm{E}-01$ & $1.483 \mathrm{E}-01$ & $2.272 E+11$ \\
\hline & 48068.000 & 1361830.00 & $1.197 \bar{E}+01$ & 5 & 3 & $1.184 \mathrm{E}-01$ & $1.483 \mathrm{E}-01$ & $2.272 \bar{E}+11$ \\
\hline$a^{1} D^{e} \quad r^{1} P^{0}$ & $\begin{array}{r}26.0981 \\
48068.000\end{array}$ & $\begin{array}{r}11.4945 \\
1650620.00\end{array}$ & $\begin{array}{l}1.460 \mathrm{E}+01 \\
1.460 \mathrm{E}+01\end{array}$ & $\begin{array}{l}5 \\
5\end{array}$ & $\begin{array}{l}3 \\
3\end{array}$ & $\begin{array}{l}3.279 \mathrm{E}-03 \\
3.279 \mathrm{E}-03\end{array}$ & $\begin{array}{l}3.368 \mathrm{E}-03 \\
3.368 \mathrm{E}-03\end{array}$ & $\begin{array}{l}9.362 \mathrm{E}+09 \\
9.362 \mathrm{E}+09\end{array}$ \\
\hline$a^{1} D^{e} \quad z^{1} D^{\circ}$ & 26.0981 & 23.2343 & $2.864 \mathrm{E}+00$ & 5 & 5 & $9.670 \mathrm{E}-02$ & $5.065 \mathrm{E}-01$ & $6.370 E+09$ \\
\hline$a^{1} D^{e}$ y ${ }^{1} D^{\circ}$ & $\begin{array}{r}48068.000 \\
26.0981\end{array}$ & $\begin{array}{r}362330.00 \\
21.9901\end{array}$ & $\begin{array}{l}2.864 \mathrm{E}+00 \\
4.108 \mathrm{E}+00\end{array}$ & $\begin{array}{l}5 \\
5\end{array}$ & $\begin{array}{l}5 \\
5\end{array}$ & $\begin{array}{l}9.670 \mathrm{E}-02 \\
4.432 \mathrm{E}-01\end{array}$ & $\begin{array}{l}5.065 \mathrm{E}-01 \\
1.618 \mathrm{E}+00\end{array}$ & $\begin{array}{l}6.370 E+09 \\
6.007 E+10\end{array}$ \\
\hline & 48068.000 & 498870.00 & $\begin{array}{l}4.108 \mathrm{E}+00 \\
4.108 \mathrm{E}+00\end{array}$ & $\begin{array}{l}5 \\
5\end{array}$ & 5 & $\begin{array}{l}4.432 \mathrm{E}=01 \\
4.432 \mathrm{E}-01\end{array}$ & $1.618 \mathrm{E}+00$ & $6.007 \mathrm{E}+10$ \\
\hline$a^{1} D^{\theta} z^{1} F^{0}$ & 26.0981 & 21.4615 & 4.637E +00 & 5 & 7 & $5.776 \mathrm{E}-01$ & $1.869 \mathrm{E}+00$ & $7.124 \mathrm{E}+10$ \\
\hline$a^{1} D^{\theta} \quad t^{1} F^{o}$ & & & & $\begin{array}{l}5 \\
5\end{array}$ & $\begin{array}{l}7 \\
7\end{array}$ & & $\begin{array}{l}1.869 \mathrm{E}+00 \\
3.996 \mathrm{E}-01\end{array}$ & $\begin{array}{l}7.124 \mathrm{E}+10 \\
4.584 \mathrm{E}+11\end{array}$ \\
\hline & 48068.000 & 1630650.00 & $1.442 \mathrm{E}+01$ & 5 & 7 & $3.842 \mathrm{E}-01$ & 3.996E-01 & $4.584 \mathrm{E}+11$ \\
\hline$h^{1} D^{e} \quad r^{1} F$ & $\begin{array}{r}12.9754 \\
488110.000\end{array}$ & $\begin{array}{r}11.4945 \\
1650620.00\end{array}$ & $\begin{array}{l}1.481 \mathrm{E}+00 \\
1.481 \mathrm{E}+00\end{array}$ & $\begin{array}{l}5 \\
5\end{array}$ & $\begin{array}{l}3 \\
3\end{array}$ & $\begin{array}{l}1.628 \mathrm{E}-02 \\
1.628 \mathrm{E}-02\end{array}$ & $\begin{array}{l}1.649 \mathrm{E}-01 \\
1.649 \mathrm{E}-01\end{array}$ & $\begin{array}{l}4.780 \mathrm{E}+08 \\
4.780 \mathrm{E}+08\end{array}$ \\
\hline $\mathrm{h}^{1} \mathrm{D}^{\mathrm{e}} \quad \mathrm{t}^{1} \mathrm{~F}^{\circ}$ & $\begin{array}{r}12.9754 \\
488110000\end{array}$ & $\begin{array}{l}11.6765 \\
30650.00\end{array}$ & $\begin{array}{l}1.299 \mathrm{E}+00 \\
1.299 \mathrm{E}+00\end{array}$ & 5 & 7 & $3.687 \mathrm{E}-01$ & $4.258 \mathrm{E}+00$ & $3.569 \mathrm{E}+09$ \\
\hline$z^{1} P^{\circ} \quad d^{1} P^{\theta}$ & $\begin{array}{l}8110.000 \\
22.5443\end{array}$ & $\begin{array}{r}30650.00 \\
12.7280\end{array}$ & $\begin{array}{l}1.299 \mathrm{E}+00 \\
9.816 \mathrm{E}+00\end{array}$ & $\begin{array}{l}5 \\
3\end{array}$ & $\begin{array}{l}7 \\
3\end{array}$ & $\begin{array}{l}E-01 \\
E-05\end{array}$ & $\begin{array}{l}4.258 \mathrm{E}+00 \\
5.332 \mathrm{E}-05\end{array}$ & $\begin{array}{l}3.569 \mathrm{E}+09 \\
4.501 \mathrm{E}+07\end{array}$ \\
\hline 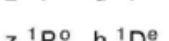 & 438050.000 & 1515260.00 & $9.816 E+00$ & 3 & 3 & $5.816 \mathrm{E}-05$ & $5.332 \mathrm{E}-05$ & $4.501 \mathrm{E}+07$ \\
\hline$z^{1} P^{\circ} \quad h^{1} D^{e}$ & $\begin{array}{r}22.5443 \\
438050.000\end{array}$ & $\begin{array}{r}12.9754 \\
1488110.00\end{array}$ & $\begin{array}{l}9.569 \mathrm{E}+00 \\
9.569 \mathrm{E}+00\end{array}$ & $\begin{array}{l}3 \\
3\end{array}$ & $\begin{array}{l}5 \\
5\end{array}$ & $\begin{array}{l}9.330 \mathrm{E}-06 \\
9.330 \mathrm{E}-06\end{array}$ & $\begin{array}{l}8.775 \mathrm{E}-06 \\
8.775 \mathrm{E}-06\end{array}$ & $\begin{array}{l}4.117 \mathrm{E}+06 \\
4.117 \mathrm{E}+06\end{array}$ \\
\hline$z^{1} P^{\circ} n^{1} D^{\theta}$ & $\begin{array}{r}22.5443 \\
8050000\end{array}$ & $\begin{array}{r}10.6728 \\
1740800.00\end{array}$ & $1.187 \mathrm{E}+01$ & 3 & 5 & $6.219 \mathrm{E}-02$ & $4.714 \mathrm{E}-02$ & 4. $224 \mathrm{E}+10$ \\
\hline$y^{1} p^{0} \quad d^{1} p^{\theta}$ & & $\begin{array}{r}1740800.00 \\
12.7280\end{array}$ & $\begin{array}{l}1.187 E+01 \\
8.608 E+00\end{array}$ & $\begin{array}{l}3 \\
3\end{array}$ & $\begin{array}{l}5 \\
3\end{array}$ & $\begin{array}{l}6.219 \mathrm{E}-02 \\
5.440 \mathrm{E}-05\end{array}$ & $\begin{array}{l}4.714 \mathrm{E}-02 \\
5.688 \mathrm{E}-05\end{array}$ & $\begin{array}{l}4.224 \mathrm{E}+10 \\
3.237 \mathrm{E}+07\end{array}$ \\
\hline $\begin{array}{l}y \\
\end{array}$ & 570690.000 & 1515260.00 & $8.608 \mathrm{E}+00$ & 3 & 3 & 5.440E-05 & $5.688 \mathrm{E}-05$ & $3.237 \mathrm{E}+07$ \\
\hline$y^{1} P^{\circ} \quad h^{1} D^{\theta}$ & $\begin{array}{r}21.3356 \\
570690.000\end{array}$ & $\begin{array}{r}12.9754 \\
1488110.00\end{array}$ & $\begin{array}{l}8.360 \mathrm{E}+00 \\
8.360 \mathrm{E}+00\end{array}$ & $\begin{array}{l}3 \\
3\end{array}$ & $\begin{array}{l}5 \\
5\end{array}$ & $\begin{array}{l}2.196 \mathrm{E}-03 \\
2.196 \mathrm{E}-03\end{array}$ & $\begin{array}{l}2.364 \mathrm{E}-03 \\
2.364 \mathrm{E}-03\end{array}$ & $\begin{array}{l}7.398 \mathrm{E}+08 \\
7.398 \mathrm{E}+08\end{array}$ \\
\hline$y^{1} P^{\circ} n^{1} D^{\theta}$ & 21.3356 & $\begin{array}{r}10.6728 \\
\end{array}$ & $1.066 \mathrm{E}+01$ & 3 & 5 & $6.704 \mathrm{E}-01$ & $5.659 \mathrm{E}-01$ & $3.674 \mathrm{E}+11$ \\
\hline$t^{1} P^{\circ} \quad d^{1} P^{e}$ & 000 & 1740800.00 & $1.066 \mathrm{E}+01$ & 3 & 5 & 6.704E-01 & $5.659 \mathrm{E}-01$ & $3.674 \mathrm{E}+11$ \\
\hline t'po d pe & $\begin{array}{r}14.1262 \\
361830.000\end{array}$ & $\begin{array}{r}12.7280 \\
1515260.00\end{array}$ & $\begin{array}{l}1.398 \mathrm{E}+00 \\
1.398 \mathrm{E}+00\end{array}$ & $\begin{array}{l}3 \\
3\end{array}$ & $\begin{array}{l}3 \\
3\end{array}$ & $\begin{array}{l}1.050 \mathrm{E}-03 \\
1.050 \mathrm{E}-03\end{array}$ & $\begin{array}{l}6.762 \mathrm{E}-03 \\
6.762 \mathrm{E}-03\end{array}$ & $\begin{array}{l}1.649 \mathrm{E}+07 \\
1.649 \mathrm{E}+07\end{array}$ \\
\hline$t^{1} P^{\circ} \quad h{ }^{1} D^{e}$ & 14.1262 & 12.9754 & $1.151 \mathrm{E}+00$ & 3 & 5 & 3.362E-01 & $2.629 \mathrm{E}+00$ & $2.145 \mathrm{E}+09$ \\
\hline$P^{\circ} n^{1} D^{e}$ & 3618 & $\begin{array}{r}1488110.00 \\
106728\end{array}$ & $1.151 \mathrm{E}+00$ & 3 & 5 & $E-01$ & $2.629 \mathrm{E}+00$ & 2.145E+09 \\
\hline$P^{0} n^{1} D^{e}$ & $\begin{array}{r}14.1262 \\
361830.000\end{array}$ & $\begin{array}{r}10.6728 \\
1740800.00\end{array}$ & $\begin{array}{l}3.453 E+00 \\
3.453 E+00\end{array}$ & $\begin{array}{l}3 \\
3\end{array}$ & $\begin{array}{l}5 \\
5\end{array}$ & $\begin{array}{l}5.080 \mathrm{E}-03 \\
5.080 \mathrm{E}-03\end{array}$ & $\begin{array}{l}1.324 \mathrm{E}-02 \\
1.324 \mathrm{E}-02\end{array}$ & $\begin{array}{l}2.920 \mathrm{E}+08 \\
2.920 \mathrm{E}+08\end{array}$ \\
\hline$r^{1} p^{\circ} n^{1} D$ & 11.4945 & 10.6728 & $\begin{array}{l}8.218 \mathrm{E}-01 \\
8.218 \mathrm{E}-01\end{array}$ & $\begin{array}{l}3 \\
3\end{array}$ & 5 & $\begin{array}{l}1.944 \mathrm{E}-01 \\
1.944 \mathrm{E}-01\end{array}$ & $\begin{array}{l}2.129 \mathrm{E}+00 \\
2.129 \mathrm{E}+00\end{array}$ & $\begin{array}{l}6.326 \mathrm{E}+08 \\
6.326 \mathrm{E}+08\end{array}$ \\
\hline$z^{1} D^{\circ} d^{1} P^{0}$ & 23.2343 & $\begin{array}{r}1740800.00 \\
12.7280\end{array}$ & $\begin{array}{l}8.218 \mathrm{E}-01 \\
1.051 \mathrm{E}+01\end{array}$ & 5 & $\begin{array}{l}5 \\
3\end{array}$ & $\begin{array}{l}1.944 \mathrm{E}-01 \\
9.473 \mathrm{E}-05\end{array}$ & $\begin{array}{l}2.129 \mathrm{E}+00 \\
1.352 \mathrm{E}-04\end{array}$ & $\begin{array}{l}6.326 \mathrm{E}+08 \\
1.400 \mathrm{E}+08\end{array}$ \\
\hline & 362330.000 & 1515260.00 & $1.051 \mathrm{E}+01$ & 5 & 3 & $9.473 \mathrm{E}-05$ & $1.352 \mathrm{E}-04$ & $1.400 \bar{E}+08$ \\
\hline$z^{1} D^{\circ} h$ & $\begin{array}{r}23.2343 \\
362330.000\end{array}$ & $\begin{array}{r}12.9754 \\
1488110.00\end{array}$ & $\begin{array}{l}1.026 \mathrm{E}+01 \\
1.026 \mathrm{E}+01\end{array}$ & $\begin{array}{l}5 \\
5\end{array}$ & $\begin{array}{l}5 \\
5\end{array}$ & $\begin{array}{l}1.201 \mathrm{E}-02 \\
1.201 \mathrm{E}-02\end{array}$ & $\begin{array}{l}1.757 \mathrm{E}-02 \\
1.757 \mathrm{E}-02\end{array}$ & $\begin{array}{l}1.016 \mathrm{E}+10 \\
1.016 \mathrm{E}+10\end{array}$ \\
\hline$z^{1} D^{\circ} n^{1} D^{\theta}$ & 23.2343 & 10.6728 & $1.256 \mathrm{E}+01$ & 5 & 5 & 2.961E-02 & $3.535 \mathrm{E}-02$ & $3.752 \mathrm{E}+10$ \\
\hline$y^{1} D^{0} d^{1} P^{\theta}$ & .000 & $\begin{array}{r}1740800.00 \\
127280\end{array}$ & 1.256E+01 & 5 & 5 & & 3.535E-02 & $2 E+10$ \\
\hline & 498870.000 & $\begin{array}{r}12.7280 \\
1515260.00\end{array}$ & $\begin{array}{l}9.262 E+00 \\
9.262 E+00\end{array}$ & $\begin{array}{l}5 \\
5\end{array}$ & $\begin{array}{l}3 \\
3\end{array}$ & $\begin{array}{l}7.506 \mathrm{E}-04 \\
7.506 \mathrm{E}-04\end{array}$ & $\begin{array}{l}1.216 \mathrm{E}-03 \\
1.216 \mathrm{E}-03\end{array}$ & $\begin{array}{l}8.620 E+08 \\
8.620 E+08\end{array}$ \\
\hline$y^{1} D^{\circ} \quad h^{1} D^{e}$ & 21.9901 & 12.9754 & $9.015 \mathrm{E}+00$ & 5 & 5 & 3. $975 \mathrm{E}-03$ & $6.614 \mathrm{E}-03$ & $2.594 \mathrm{E}+09$ \\
\hline$y^{1} D^{\circ} n^{1} D^{e}$ & & $\begin{array}{r}88110.00 \\
10.6728\end{array}$ & $\begin{array}{l}9.015 \mathrm{E}+00 \\
1.132 \mathrm{E}+01\end{array}$ & $\begin{array}{l}5 \\
5\end{array}$ & $\begin{array}{l}5 \\
5\end{array}$ & $\begin{array}{l}3.975 \mathrm{E}-03 \\
3.959 \mathrm{E}-02\end{array}$ & $\begin{array}{l}6.614 \\
5.247\end{array}$ & $\begin{array}{l}2.594 \mathrm{E}+09 \\
4.073 \mathrm{E}+10\end{array}$ \\
\hline & 498870.000 & 1740800.00 & $1.132 \mathrm{E}+01$ & 5 & 5 & 3.959 & 5.247 & $4.073 \mathrm{E}+10$ \\
\hline$z^{1} F^{\circ} \quad h{ }^{1} D^{e}$ & 21.4615 & 12.9754 & $8.486 E+00$ & 7 & 5 & 3.059E-02 & $7.570 \mathrm{E}-02$ & $2.477 \mathrm{E}+10$ \\
\hline$=0$ & $\begin{array}{r}6870.000 \\
21.4615\end{array}$ & $\begin{array}{r}14881 \\
10\end{array}$ & & $\begin{array}{l}7 \\
7\end{array}$ & $\begin{array}{l}5 \\
5\end{array}$ & & $\begin{array}{l}7.5 \\
21\end{array}$ & $\begin{array}{l}+10 \\
+09\end{array}$ \\
\hline$z$ & $\begin{array}{r}21.4615 \\
556870.000\end{array}$ & $\begin{array}{r}10.6 / 28 \\
1740800.00\end{array}$ & $\begin{array}{l}1.0 / 9 \mathrm{E}+01 \\
1.079 \mathrm{E}+01\end{array}$ & 7 & $\begin{array}{l}5 \\
5\end{array}$ & $\begin{array}{l}1.129 \mathrm{E}-03 \\
1.129 \mathrm{E}-03\end{array}$ & $\begin{array}{l}2.197 \mathrm{E}-03 \\
2.197 \mathrm{E}-03\end{array}$ & $\begin{array}{l}1.471 \mathrm{E}+09 \\
1.477 \mathrm{E}+09\end{array}$ \\
\hline$z^{1} F^{\circ} g^{1} G^{e}$ & $\begin{array}{r}21.4615 \\
556870.000\end{array}$ & $\begin{array}{r}10.6485 \\
1743460.00\end{array}$ & $\begin{array}{l}1.081 \mathrm{E}+01 \\
1.081 \mathrm{E}+01\end{array}$ & 7 & $\begin{array}{l}9 \\
9\end{array}$ & $\begin{array}{l}7.214 \mathrm{E}-01 \\
7.214 \mathrm{E}-01\end{array}$ & $\begin{array}{l}1.401 \mathrm{E}+00 \\
1.401 \mathrm{E}+00\end{array}$ & $\begin{array}{l}5.270 \mathrm{E}+11 \\
5.270 \mathrm{E}+11\end{array}$ \\
\hline$t^{1} F^{\circ} n^{1} D^{e}$ & 11.6765 & 10.6728 & $1.004 \mathrm{E}+00$ & 7 & 5 & $2.038 \mathrm{E}-03$ & 4.265E-02 & $2.310 E+07$ \\
\hline & $\begin{array}{r}630650.000 \\
11.6765\end{array}$ & 1740800.00 & $1.004 \mathrm{E}+00$ & 7 & 5 & & 4.265E-02 & $2.310 E+07$ \\
\hline $7+$ & $\begin{array}{r}11.6765 \\
630650.000\end{array}$ & $\begin{array}{r}10.6485 \\
1743460.00\end{array}$ & $\begin{array}{l}1.028 \mathrm{E}+00 \\
1.028 \mathrm{E}+00\end{array}$ & 7 & $\begin{array}{l}9 \\
9\end{array}$ & $\begin{array}{l}2.550 \mathrm{E}-01 \\
2.550 \mathrm{E}-01\end{array}$ & $\begin{array}{l}5.209 \mathrm{E}+00 \\
5.209 \mathrm{E}+00\end{array}$ & $\begin{array}{l}1.683 \mathrm{E}+09 \\
1.683 \mathrm{E}+09\end{array}$ \\
\hline$a^{3} P^{e} \quad z^{3} S^{0}$ & $\begin{array}{r}26.4139 \\
18561.000\end{array}$ & $\begin{array}{r}22.7501 \\
415462.00\end{array}$ & $\begin{array}{l}3.664 \mathrm{E}+00 \\
3.617 \mathrm{E}+00\end{array}$ & $\begin{array}{l}9 \\
5\end{array}$ & $\begin{array}{l}3 \\
3\end{array}$ & $\begin{array}{l}1.961 \mathrm{E}-01 \\
1.936 \mathrm{E}-01\end{array}$ & $\begin{array}{l}1.445 \mathrm{E}+00 \\
8.028 \mathrm{E}-01\end{array}$ & $\begin{array}{l}6.342 \mathrm{E}+10 \\
3.390 \mathrm{E}+10\end{array}$ \\
\hline
\end{tabular}

TABLE II. Oscillator Strengths, Line Strengths, and Transition Probabilities for Allowed Transitions in Fe XIII 
See page 137 for Explanation of Tables

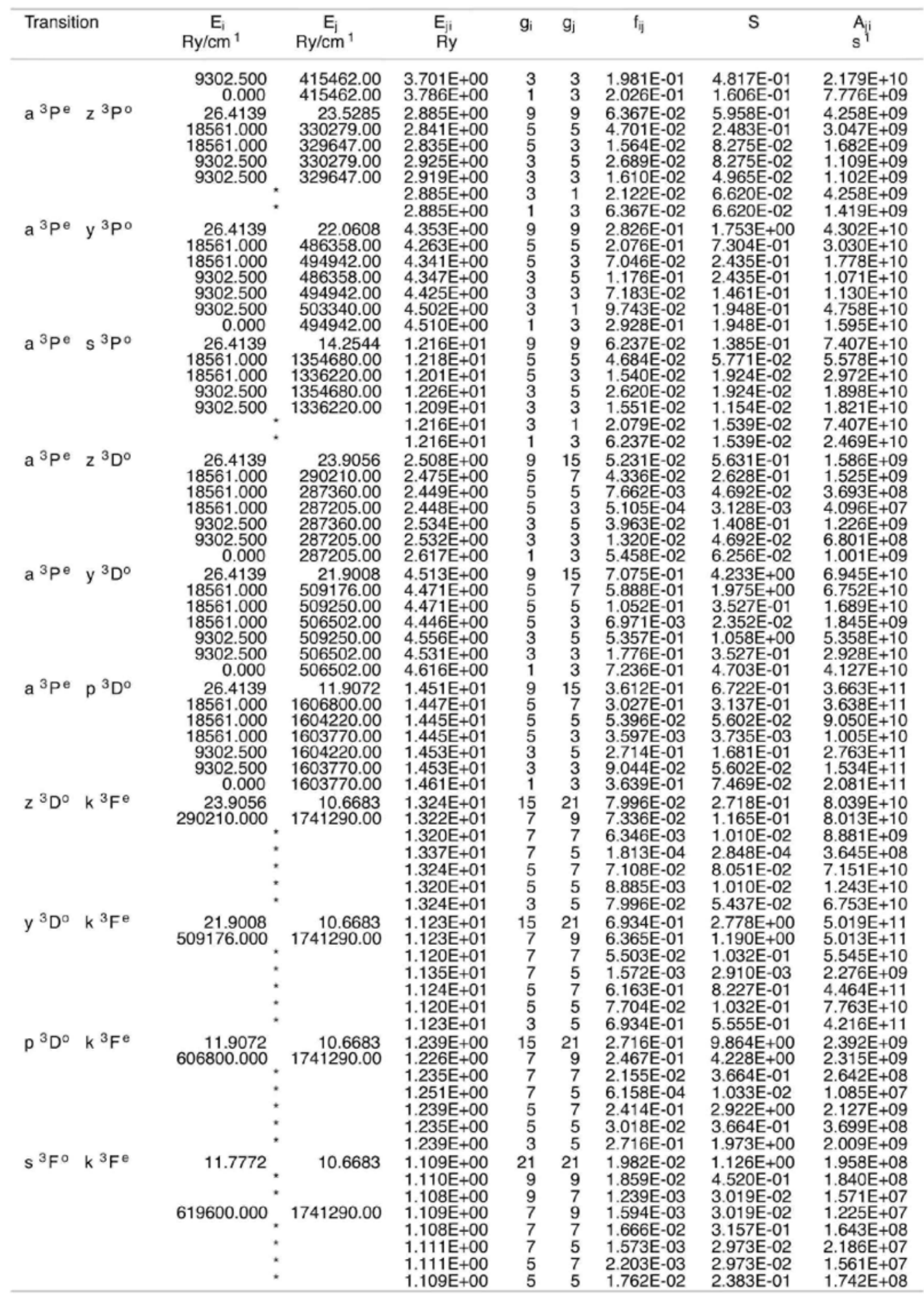

